\title{
A Sterically Expanded "Constrained Geometry Catalyst" for Highly Active Olefin Polymerization and Copolymerization: An Unyielding Comonomer Effect
}

Levi J. Irwin, Joseph H. Reibenspies, and Stephen A. Miller*

Department of Chemistry, Texas A\&M University

College Station, Texas 77843-3255

\section{Supporting Information}

Supporting Information Available: Synthesis and characterization data for $\mathbf{1}$ as well as polymerization details and polymer characterization data.

\section{Table of Contents}

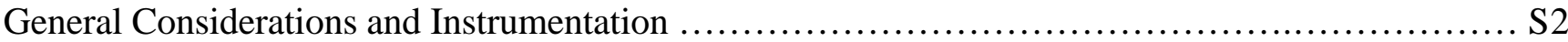

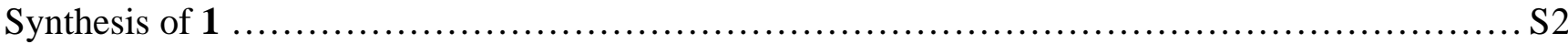

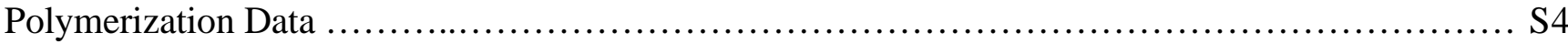

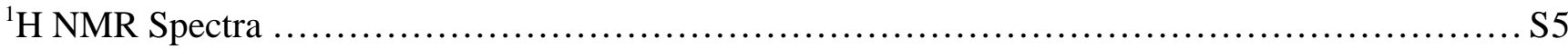

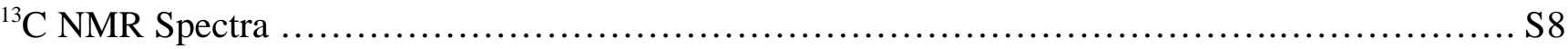

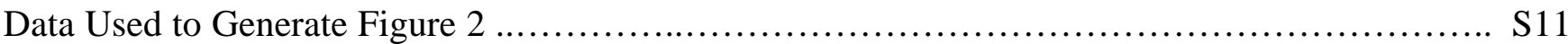

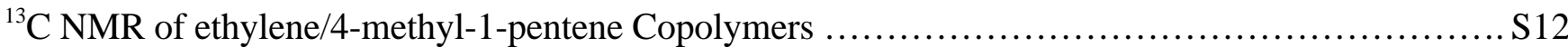

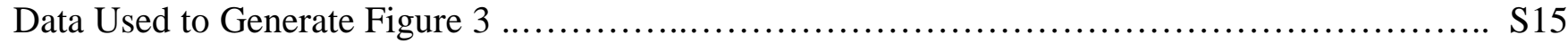

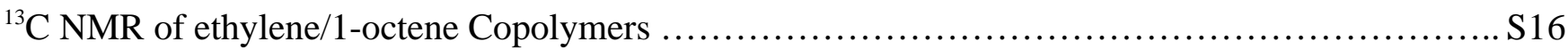

X-Ray Structure Data for octamethyloctahydrodibenzofluorene $(\operatorname{sm} 34) \ldots \ldots \ldots \ldots \ldots \ldots \ldots \ldots \ldots . . \ldots \ldots$

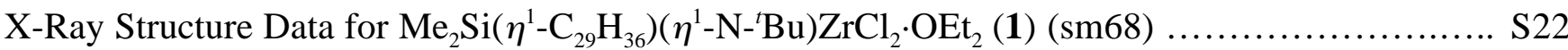

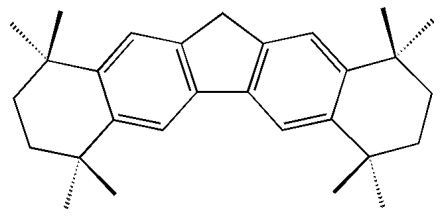

octamethyloctahydrodibenzofluorene

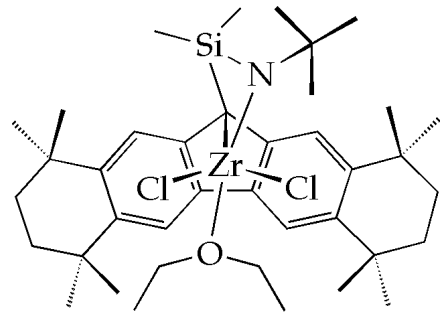

$\mathrm{Me}_{2} \mathrm{Si}\left(\eta^{1}-\mathrm{C}_{29} \mathrm{H}_{36}\right)\left(\eta^{1}-\mathrm{N}-{ }^{t} \mathrm{Bu}\right) \mathrm{ZrCl}_{2} \cdot \mathrm{OEt}_{2}(\mathbf{1})$ 
General Considerations and Instrumentation. All air sensitive procedures were carried out under a purified atmosphere of nitrogen in a glove box equipped with a $-35^{\circ} \mathrm{C}$ freezer or by using standard Schlenk line techniques. Solvents were dried and distilled under nitrogen into Straus flasks and stored until needed. Diethyl ether and tetrahydrofuran were distilled from sodium/benzophenone, hexanes and dichloromethane were distilled from calcium hydride, and toluene was distilled from elemental sodium. The commercially available reagents 2,5-dimethyl-2,5hexanediol (TCI, 98\%), fluorene (Acros, 98\%), aluminum trichloride (Strem, 99\%), n-butyllithium butyllithium (Acros, 2.5 $\mathrm{M}$ in hexanes or Alfa Aesar, 2.87 $\mathrm{M}$ in hexanes), dichlorodimethylsilane (Acros, 99+\%), $t$ butylamine (Acros, 99\%), and zirconium tetrachloride (Strem, 99.5+\%) were used as received, unless otherwise noted. $\mathrm{Me}_{2} \mathrm{Si}\left(\eta^{5}-\mathrm{C}_{5} \mathrm{Me}_{4}\right)\left(\eta^{1}-\mathrm{N}-{ }^{t} \mathrm{Bu}\right) \mathrm{TiCl}_{2}(2)$ was acquired as a gift from The Dow Chemical Company and used as received. MAO was acquired from Albemarle as a $30 \mathrm{wt} \%$ solution in toluene and prepared by drying under high vacuum at $70^{\circ} \mathrm{C}$ for 3 days. Both 4-methyl-1-pentene (TCI, 97\%) and 1-octene (ICN, 98\%) were vacuum transferred from calcium hydride into Straus flasks where they were degassed with three freeze-pump-thaw cycles and then stored in a glove box. All NMR chemical shifts are given in ppm and were recorded on a Mercury-300BB spectrometer $\left({ }^{1} \mathrm{H}, 299.91 \mathrm{MHz} ;{ }^{13} \mathrm{C}\left\{{ }^{1} \mathrm{H}\right\}, 75.41 \mathrm{MHz}\right)$ using the solvent peak (or residual protonated solvent peak) as an internal standard $\left(\mathrm{CDCl}_{3}:{ }^{1} \mathrm{H}, 7.27 \mathrm{ppm} ;{ }^{13} \mathrm{C} 77.0 \mathrm{ppm} . \mathrm{C}_{6} \mathrm{D}_{6}:{ }^{1} \mathrm{H}\right.$, $\left.7.15 \mathrm{ppm},{ }^{13} \mathrm{C} 128.0 \mathrm{ppm}\right)$.

2,5-Dichloro-2,5-dimethylhexane $\quad\left(\mathrm{C}_{8} \mathrm{H}_{16} \mathrm{Cl}_{2}\right)$. A 4 liter Erlenmeyer flask was charged with 2,5-dimethyl2,5-hexanediol (400.00 g, $2.735 \mathrm{~mol})$ and concentrated aqueous hydrochloric acid $(2.00 \mathrm{~L}, 24.4 \mathrm{~mol})$ was poured in. The white slurry was shaken and allowed to stand for three days. The white solid was then dissolved in $2.00 \mathrm{~L}$ of diethyl ether. The aqueous phase was separated and extracted with diethyl ether $(2 \times 100 \mathrm{~mL})$. The combined organics were then split into two batches and each washed with water $(3 \times 100 \mathrm{~mL})$, dried with $\mathrm{MgSO}_{4}$, and filtered. The organics were concentrated by rotary evaporation, yielding a white slurry (approximately $300 \mathrm{~mL}$ ). This was heated in a warm water bath until homogeneous and allowed to cool slowly to room temperature and finally cooled in the freezer to $0^{\circ} \mathrm{C}$. Collection of two crops by suction filtration followed by air-drying yielded the product as large plate-like clear crystals: $446.99 \mathrm{~g}(89.4 \%) . \quad{ }^{1} \mathrm{H}$ $\operatorname{NMR}\left(\mathrm{CDCl}_{3}\right): \delta 1.58\left(\mathrm{~s}, 12 \mathrm{H}, \mathrm{CH}_{3}\right), 1.93\left(\mathrm{~s}, 4 \mathrm{H}, \mathrm{CH}_{2}\right)$. ${ }^{13} \mathrm{C}\left\{{ }^{1} \mathrm{H}\right\}$ NMR $\left(\mathrm{CDCl}_{3}\right): \delta 32.9,41.4,70.4$. GC MS: $\mathrm{m} / z$ $147(\mathrm{M}-\mathrm{Cl})^{+}$.

Octamethyloctahydrodibenzofluorene $\left(\mathrm{C}_{29} \mathrm{H}_{38}\right)$. A 2 liter round bottom flask was charged with fluorene $(90.77 \mathrm{~g}, 546.10 \mathrm{mmol}), 1.00 \mathrm{~L}$ of nitromethane and 2,5dichloro-2,5-dimethylhexane (200.00 g, $1.09 \mathrm{~mol})$. The round bottom was then sealed with a septum and attached to an oil bubbler. The slurry was warmed in a water bath and purged for 20 minutes with nitrogen. Meanwhile, in the glove box, a $300 \mathrm{~mL}$ round bottom flask was charged with $\mathrm{AlCl}_{3}(95.16 \mathrm{~g}, 714.2 \mathrm{mmol})$ and sealed with a septum. This was then brought out of the glove box and $60 \mathrm{~mL}$ of nitromethane were slowly syringed in. Once the $\mathrm{AlCl}_{3}$ was dissolved, the solution was slowly syringed over a period of 40 minutes into the homogeneous solution in the 2 liter round bottom flask. The solution immediately became ink blue, a precipitate formed, and evolution of $\mathrm{HCl}$ gas was confirmed by $\mathrm{pH}$ paper. After stirring the slurry for 19 hours, it was slowly dumped into $1.00 \mathrm{~L}$ of ice water and the solid was collected by suction filtration. The light green solid was then triturated in $500 \mathrm{~mL}$ ethanol overnight, filtered, and slowly recrystallized from boiling toluene to yield the product as off-white needle-like crystals: $187.01 \mathrm{~g}(88.6 \%)$. Crystals suitable for X-ray diffraction were grown by vapor diffusion of diethyl ether into a saturated dichloromethane solution. ${ }^{1} \mathrm{H}$ NMR $\left(\mathrm{CDCl}_{3}\right): \delta 1.34\left(\mathrm{~s}, 12 \mathrm{H}, \mathrm{CH}_{3}\right), 1.40\left(\mathrm{~s}, 12 \mathrm{H}, \mathrm{CH}_{3}\right), 1.74$ (apparent s, $\left.8 \mathrm{H}, \mathrm{CH}_{2}\right), 3.78\left(\mathrm{~s}, 2 \mathrm{H}, \mathrm{Ar}_{2} \mathrm{CH}_{2}\right), 7.45,7.67$ (s, $\left.4 \mathrm{H}, \mathrm{CH}_{1}\right) .{ }^{13} \mathrm{C}\left\{{ }^{1} \mathrm{H}\right\}$ NMR $\left(\mathrm{CDCl}_{3}\right): \delta 32.6,32.7,34.93$, $34.95,35.7,35.8,36.6,117.6,123.2,139.8,141.2$, 143.5, 143.6. GC MS: $m / z 387(\mathrm{M}+\mathrm{H})^{+}$.

$\operatorname{ClSi}\left(\mathrm{CH}_{3}\right)_{2}\left(\mathbf{C}_{29} \mathbf{H}_{37}\right)$. A $500 \mathrm{~mL}$ round bottom was charged with $20.00 \mathrm{~g} \quad(51.73 \mathrm{mmol})$ of octamethyloctahydrodibenzofluorene and a $180^{\circ}$ needle valve was attached. The system was evacuated and dry THF (200 $\mathrm{mL}$ ) was vacuum transferred in. Next, $n$-butyllithium $(19.83 \mathrm{~mL}, 56.90 \mathrm{mmol}, 2.87 \mathrm{M}$ in hexanes) was syringed in over five minutes and the red slurry was stirred for 20 hours. Meanwhile, a $7 \mathrm{~cm}$ swivel frit was assembled with two $500 \mathrm{~mL}$ round bottom flasks and evacuated. Hexanes $(100 \mathrm{~mL})$ were then vacuum transferred into the frit followed by an excess of $\left(\mathrm{CH}_{3}\right)_{2} \mathrm{SiCl}_{2}(150 \mathrm{~mL}, 1230$ mmol, dried over $\mathrm{CaH}_{2}$ ). Next, the THF was removed from the lithium salt $\mathrm{C}_{29} \mathrm{H}_{37} \mathrm{Li}$ prepared earlier and hexanes vacuum transferred in. This red slurry was then cannulated directly into the $7 \mathrm{~cm}$ frit over 15 minutes, resulting in a white slurry. After 17.5 hours, the hexanes and excess $\left(\mathrm{CH}_{3}\right)_{2} \mathrm{SiCl}_{2}$ were removed under vacuum and diethyl ether $(150 \mathrm{~mL})$ was vacuum transferred in. The $\mathrm{LiCl}$ was removed by filtration and the ether was removed under vacuum. The product was collected in the glove box as an off-white solid: $21.73 \mathrm{~g}(87.7 \%)$. ${ }^{1} \mathrm{H}$ NMR $\left(\mathrm{C}_{6} \mathrm{D}_{6}\right): \delta \quad 0.03$ $\left(\mathrm{s}, 6 \mathrm{H},\left(\mathrm{CH}_{3}\right)_{2} \mathrm{Si}\right), 1.32,1.33,1.34,1.39(\mathrm{~s}, 24 \mathrm{H}$, Oct$\mathrm{CH}_{3}$ ), 1.66 (apparent s, 8H, Oct- $\left.\mathrm{CH}_{2}\right), 3.85(\mathrm{~s}, 1 \mathrm{H}$, $\left.\mathrm{Ar}_{2} \mathrm{CH}_{1}\right), 7.71,8.02$ (s, 4H, Oct- $\left.\mathrm{CH}_{1}\right) .{ }^{13} \mathrm{C}\left\{{ }^{1} \mathrm{H}\right\}$ NMR $\left(\mathrm{C}_{6} \mathrm{D}_{6}\right): \quad \delta-0.3,32.36,32.44,32.44,32.56,34.65,34.8$, $35.5,35.7,42.5,117.6,123.2,139.5,141.0,143.2$, 143.3. TOF MS/ESI: $m / z$ 495.29 (M+OH), 385.29 $\left(\mathrm{C}_{29} \mathrm{H}_{37}\right)^{+}$.

$\left(\mathrm{CH}_{3}\right)_{3} \mathrm{CNH}\left(\mathrm{CH}_{3}\right)_{2} \mathrm{Si}\left(\mathrm{C}_{29} \mathrm{H}_{37}\right)$. In the glove box $\mathrm{ClSi}\left(\mathrm{CH}_{3}\right)_{2}\left(\mathrm{C}_{29} \mathrm{H}_{37}\right)(37.19 \mathrm{~g}, 77.60 \mathrm{mmol})$ was combined with $\left(\mathrm{CH}_{3}\right)_{3} \mathrm{CNHLi}(6.14 \mathrm{~g}, 77.60 \mathrm{mmol})$ (made by addition of $60.0 \mathrm{~mL}$ of $n$-butyllithium $(138.6 \mathrm{mmol})$ in hexanes to $9.22 \mathrm{~g}(126.0 \mathrm{mmol})$ of $t$-butylamine in hexanes at room temperature, collected by filtration and dried in vacuo) in a $500 \mathrm{~mL}$ round bottom flask and attached to a $7 \mathrm{~cm}$ swivel frit. The frit was then evacuated and diethyl ether $(200 \mathrm{~mL})$ was vacuum transferred in. The round bottom was allowed to warm slowly to room temperature resulting in the formation of an off-white suspension that was slowly gravity filtered for 12 hours, thereby removing the $\mathrm{LiCl}$. The ether was removed under vacuum and the product was collected in the glove box yielding $33.70 \mathrm{~g}(84.3 \%)$ of an off-white solid. ${ }^{1} \mathrm{H}$ NMR $\left(\mathrm{CDCl}_{3}\right): \delta-0.11\left(\mathrm{~s}, 6 \mathrm{H},\left(\mathrm{CH}_{3}\right)_{2} \mathrm{Si}\right), 0.61\left(\mathrm{~s}, 1 \mathrm{H}, \mathrm{NH}_{1}\right)$, $1.20\left(\mathrm{~s}, 9 \mathrm{H},-\mathrm{C}\left(\mathrm{CH}_{3}\right)_{3}\right), 1.32,1.34,1.37,1.38(\mathrm{~s}, 24 \mathrm{H}$, Oct- $\left.\mathrm{CH}_{3}\right), 1.73$ (apparent s, $8 \mathrm{H}$, Oct- $\left.\mathrm{CH}_{2}\right), 3.68(\mathrm{~s}, 1 \mathrm{H}$, $\mathrm{Ar}_{2} \mathrm{CH}_{1}$ ), 7.48, 7.68 (s, 4H, Oct- $\left.\mathrm{CH}_{1}\right) .{ }^{13} \mathrm{C}\left\{{ }^{1} \mathrm{H}\right\}$ NMR $\left(\mathrm{C}_{6} \mathrm{D}_{6}\right): \delta-0.1,32.54,32.55,32.59,32.8,34.0,34.6$, 
34.9, 35.8, 35.9, 43.6, 49.6, 117.3, 122.7, 139.5, 142.0, 142.5, 143.8. TOF MS/ESI: $m / z 516.40(\mathrm{M}+\mathrm{H})^{+}$.

$\mathrm{Me}_{2} \mathrm{Si}\left(\mathrm{C}_{29} \mathrm{H}_{36}\right)\left(\mathrm{N}^{t}{ }^{t} \mathbf{B u}\right) \mathrm{ZrCl}_{2} \cdot \mathbf{O E t}_{2}$ (1). In the glove box $\left(\mathrm{CH}_{3}\right)_{3} \mathrm{CNH}\left(\mathrm{CH}_{3}\right)_{2} \mathrm{Si}\left(\mathrm{C}_{29} \mathrm{H}_{37}\right)(3.00 \mathrm{~g}, 6.26 \mathrm{mmol})$ was charged into a $3 \mathrm{~cm}$ swivel frit. The frit was then evacuated and diethyl ether $(50 \mathrm{~mL})$ was vacuum transferred in. Next, $n$-butyllithium (5.51 mL, $13.77 \mathrm{mmol}, 2.5 \mathrm{M}$ in hexanes) was syringed in. The orange slurry was stirred for 20 hours and then the diethyl ether was removed under vacuum. $\mathrm{ZrCl}_{4}(1.46 \mathrm{~g}, 6.26 \mathrm{mmol})$ was added in the glove box and diethyl ether $(40 \mathrm{~mL})$ vacuum transferred in on the line. After slowly warming to room temperature and stirring the light brown slurry for 48 hours, the $\mathrm{LiCl}$ was filtered off and the cake extracted until colorless. The slurry was concentrated to about $10 \mathrm{~mL}$ and the precipitated solid was collected by filtration. The cake was washed once to remove a brown oil and then evacuated until dry. This yielded $1.42 \mathrm{~g}(31.8 \%)$ of product as a neon yellow solid. Large rod-shaped crystals can be grown by cooling a saturated diethyl ether solution to $-35^{\circ} \mathrm{C}$. ${ }^{1} \mathrm{H}$ NMR $\left(\mathrm{C}_{6} \mathrm{D}_{6}\right)$ : $\delta 0.86\left(\mathrm{~s}, 6 \mathrm{H},\left(\mathrm{CH}_{3}\right)_{2} \mathrm{Si}\right), 1.10\left(\mathrm{t},{ }^{3} J_{\mathrm{HH}}=7.0 \mathrm{~Hz}, 6 \mathrm{H}\right.$, ether$\left.\mathrm{CH}_{3}\right), 1.26\left(\mathrm{~s}, 9 \mathrm{H},-\mathrm{C}\left(\mathrm{CH}_{3}\right)_{3}\right), 1.28,1.35,1.38,1.39$ (s, $24 \mathrm{H}$, Oct- $\left.\mathrm{CH}_{3}\right), 1.62\left(\mathrm{~m}, 8 \mathrm{H}\right.$, Oct- $\left.\mathrm{CH}_{2}\right), 3.25$ (q, ${ }^{3} J_{\mathrm{HH}}=7.0$ $\mathrm{Hz}, 4 \mathrm{H}$, ether- $\left.\mathrm{CH}_{2}\right), 8.01,8.24\left(\mathrm{~s}, 4 \mathrm{H}\right.$, Oct- $\left.\mathrm{CH} H_{1}\right) .{ }^{13} \mathrm{C}\left\{{ }^{1} \mathrm{H}\right\}$ NMR $\left(\mathrm{C}_{6} \mathrm{D}_{6}\right): \delta 5.9,15.5,32.3,32.6,32.8,32.9,33.4$, $34.95,35.02$, 35.3, 35.4, 56.4, 66.0, 121.5, 123.2, 125.3, 127.5, 134.8, 145.6, 148.9. Elemental Analysis: Calculated: C, 62.44\%; H, 8.13\%; N, 1.87\%; Cl, 9.45\%. Found: C, 61.40\%; H, 8.24\%; N, 1.81\%; Cl, 9.45\%.

Polymerizations: CAUTION: All polymerizations should be carried out in a fume hood behind a blast shield. Olefin polymerizations were carried out in an $85 \mathrm{~mL}$ glass Lab-Crest ${ }^{\circledR}$ (Andrews Glass Co.) cylindrical polymerization reactor equipped with a 2 inch cylindrical stir bar able to provide ample surface agitation while stirring. The apparatus was brought into the box where the reactor was charged with $0.100 \mathrm{~g}$ solid MAO $\left(1.66 \times 10^{-3} \mathrm{~mol}, 1000\right.$ equivalents, prepared as described above) and $25 \mathrm{~mL}$ of the required $\alpha$-olefin or $25 \mathrm{~mL}$ of toluene for the ethylene homopolymerizations. In the case of the 1-octene/4methyl-1-pentene copolymerization, $13.0 \mathrm{~mL} \mathrm{1-octene} \mathrm{and}$ $12.0 \mathrm{~mL}$ of 4-methyl-1-pentene were added in the box. Next, $5 \mathrm{~mL}$ of a stock solution (previously prepared by dissolving $0.250 \mathrm{~g}$ of 1 in $100 \mathrm{~mL}$ toluene) was transferred to a $10 \mathrm{~mL}$ volumetric flask and the contents brought to mark with toluene. Finally, $1.0 \mathrm{~mL}\left(1.66 \times 10^{-6} \mathrm{~mol} \mathrm{1}\right.$, assuming mono-ether adduct) was drawn from the $10 \mathrm{~mL}$ volumetric flask into a $2.5 \mathrm{~mL}$ Hamilton Gastight syringe and the tip imbedded into a septum. The reactor was then brought out of the box and placed in a $75^{\circ} \mathrm{C}$ oil bath and, if necessary, the designated pressure of ethylene (80 psi $\sim 0.5$ M) was applied. The system was then allowed to equilibrate for at least 10 minutes with vigorous stirring. The solution of $\mathbf{1}$ was then syringed in and there was an immediate color change from neon yellow to bright orange. The polymerizations were run for 50 seconds before being quickly vented and $4 \mathrm{~mL}$ of a $5 \%$ aqueous $\mathrm{HCl}$ in $\mathrm{MeOH}$ solution syringed directly into the reactor. The contents of the rector were then combined with $150 \mathrm{~mL}$ of $5 \%$ aqueous $\mathrm{HCl}$ in $\mathrm{MeOH}$ in a tared beaker. A stir bar was massed and then added. The contents were stirred vigorously allowing the MAO to be thoroughly quenched. Next, the polymer was collected by decanting the $\mathrm{HCl} / \mathrm{MeOH}$ solution from the polymer and then rinsing several times with fresh methanol-or in the case of the ethylene or poly(4-methyl1-pentene) homopolymers, the polymers were collected by filtration and washed at least 3 times with fresh methanol. The waxy polymers were then dried at ambient temperature for 3 days under a high vacuum polycarbonate dome. Every 12 hours the waxes were stirred with individually designated spatulas (previously massed) to maximize exposure to vacuum. The mass of the polymer was then determined by subtracting the mass of the beaker, stir bar, and spatula from the total mass. Typical polymer yields were between 0.2 and 12 grams. See the summary data below.

An identical polymerization procedure was carried out with $\mathrm{Me}_{2} \mathrm{Si}\left(\eta^{5}-\mathrm{C}_{5} \mathrm{Me}_{4}\right)\left(\eta^{1}-\mathrm{N}-{ }^{t}{ }^{-} \mathrm{uu}\right) \mathrm{TiCl}_{2}$ (2) using a solution prepared by diluting $4 \mathrm{~mL}$ of a stock solution (previously prepared by dissolving $0.150 \mathrm{~g} 2$ in $100 \mathrm{~mL}$ toluene) to 10 $\mathrm{mL}$. One $\mathrm{mL}\left(1.63 \times 10^{-6} \mathrm{~mol} \mathrm{2}\right)$ of this solution was then drawn into a $2.5 \mathrm{~mL}$ Hamilton Gastight syringe and used in the polymerizations.

Only a small amount of polymer was produced in the $\alpha$ olefin homopolymerizations and the 1-octene/4-methyl-1pentene copolymerization with 2/MAO. As a consequence, the masses were acquired by quenching the contents of the reactor with $4 \mathrm{~mL} 5 \%$ aqueous $\mathrm{HCl}$ in $\mathrm{MeOH}$ and then pouring the contents into a tared $500 \mathrm{~mL}$ round bottom flask. All volatiles were removed under high vacuum and the solids then washed with $5 \% \mathrm{HCl}$ in water followed by a rinse with distilled water. The film of polymer was then dried under vacuum and the mass recorded.

${ }^{13} \mathrm{C}$ NMR of both the ethylene/4-methyl-1-pentene copolymers and ethylene/1-octene copolymers were recorded on a Unity-Inova $300 \mathrm{MHz}$ NMR operating at $74.42 \mathrm{MHz}$. The delay times and acquisition times were each set to 2 seconds. ${ }^{1}$ Ethylene/4-methyl-1-pentene copolymers were measured at $130^{\circ} \mathrm{C}$ in a $3: 1$ solution of $1,2,4-$ trichlorobenzene and 1,1,2,2-tetrachloroethane- $d_{2} .{ }^{13} \mathrm{C}$ NMR of ethylene/1-octene copolymers were recorded at $130^{\circ} \mathrm{C}$ in 1,1,2,2-tetrachloroethane- $d_{2}$. The relative quantification of ethylene and $\alpha$-olefin was performed according to the method of Kimura. ${ }^{2}$

\section{References}

(1) Xu, G.; Cheng, D. Macromolecules 2001, 34, 2040-2047.

(2) Kimura, K.; Yuasa, S.; Maru, Y. Polymer 1984, 25, 441-446. 


\section{Polymerization Data}

Table 1. Polymerization and copolymerization activities with $\mathbf{1} / \mathrm{MAO}{ }^{a}$

\begin{tabular}{|c|c|c|c|c|c|c|c|c|c|c|}
\hline $\begin{array}{c}\text { Monomer } \\
1 \\
\end{array}$ & Amount & $\begin{array}{c}\begin{array}{c}\text { Volume } \\
(\mathrm{mL})\end{array} \\
\end{array}$ & $\begin{array}{c}\text { Monomer } \\
2 \\
\end{array}$ & Amount & $\begin{array}{c}\begin{array}{c}\text { Volume } \\
\text { (mL) }\end{array} \\
\end{array}$ & $\begin{array}{l}\text { Time } \\
(\min .)\end{array}$ & $\begin{array}{c}\text { Temp. } \\
\left({ }^{\circ} \mathrm{C}\right)\end{array}$ & $\begin{array}{c}\text { Total } \\
\text { Toluene }(\mathrm{mL}) \\
\end{array}$ & $\begin{array}{c}\text { Activity } \\
\mathrm{kg} /(\mathrm{mol} \mathrm{Zr} \cdot \mathrm{h})\end{array}$ & $\begin{array}{l}\text { Yield } \\
(\mathrm{g})\end{array}$ \\
\hline ethylene & $0.5 \mathrm{M}$ & - & - & - & - & 1.0 & 75 & 26.0 & 3,290 & 0.091 \\
\hline ethylene & $0.5 \mathrm{M}$ & - & - & - & - & 1.0 & 75 & 26.0 & 4,880 & 0.135 \\
\hline ethylene & $0.5 \mathrm{M}$ & - & - & - & - & 1.0 & 75 & 26.0 & 4,270 & 0.118 \\
\hline $\begin{array}{l}\text { 4-methyl- } \\
\text { 1-pentene }\end{array}$ & $7.54 \mathrm{M}$ & 25.0 & - & - & - & 1.0 & 75 & 1.0 & 27,830 & 0.770 \\
\hline 1-octene & $6.17 \mathrm{M}$ & 25.0 & - & - & - & 1.0 & 75 & 1.0 & 316,080 & 8.745 \\
\hline $\begin{array}{l}\text { 4-methyl- } \\
1 \text {-pentene }\end{array}$ & $7.54 \mathrm{M}$ & 25.0 & ethylene & $0.5 \mathrm{M}$ & - & 1.0 & 75 & 1.0 & 209,280 & 5.790 \\
\hline 1-octene & $6.17 \mathrm{M}$ & 25.0 & ethylene & $0.5 \mathrm{M}$ & - & 1.0 & 75 & 1.0 & 437,710 & 12.110 \\
\hline $\begin{array}{l}\text { 4-methyl- } \\
\text { 1-pentene }\end{array}$ & $3.62 \mathrm{M}$ & 12.0 & 1-octene & $3.19 \mathrm{M}$ & 13.0 & 1.0 & 75 & 1.0 & 166,050 & 4.594 \\
\hline
\end{tabular}

${ }^{\mathrm{a}} 1.66 \mu \mathrm{mol} 1$ dissolved in $1.0 \mathrm{~mL}$ toluene; 1000 equivalents MAO.

Table 2. Polymerization and copolymerization activities with 2/MAO. ${ }^{a}$

\begin{tabular}{|c|c|c|c|c|c|c|c|c|c|c|}
\hline $\begin{array}{c}\text { Monomer } \\
1\end{array}$ & Amount & $\begin{array}{l}\text { Volume } \\
\text { (mL) }\end{array}$ & $\begin{array}{c}\text { Monomer } \\
2\end{array}$ & Amount & $\begin{array}{l}\text { Volume } \\
\text { (mL) }\end{array}$ & $\begin{array}{l}\text { Time } \\
(\min .)\end{array}$ & $\begin{array}{l}\text { Temp. } \\
\left({ }^{\circ} \mathrm{C}\right)\end{array}$ & $\begin{array}{c}\text { Total } \\
\text { Toluene }(\mathrm{mL})\end{array}$ & $\begin{array}{c}\text { Activity } \\
\mathrm{kg} /(\text { mol Zr} \cdot \mathrm{h})\end{array}$ & $\begin{array}{l}\text { Yield } \\
(\mathrm{g})\end{array}$ \\
\hline ethylene & $0.5 \mathrm{M}$ & - & - & - & - & 0.5 & 75 & 26.0 & 26,130 & 0.355 \\
\hline ethylene & $0.5 \mathrm{M}$ & - & - & - & - & 0.5 & 75 & 26.0 & 26,800 & 0.364 \\
\hline ethylene & $0.5 \mathrm{M}$ & - & - & - & - & 0.5 & 75 & 26.0 & 26,800 & 0.364 \\
\hline $\begin{array}{l}\text { 4-methyl- } \\
\text { 1-pentene }\end{array}$ & $7.54 \mathrm{M}$ & 25.0 & - & - & - & 1.0 & 75 & 1.0 & 2,940 & 0.080 \\
\hline 1-octene & $6.17 \mathrm{M}$ & 25.0 & - & - & - & 1.0 & 75 & 1.0 & 3,680 & 0.100 \\
\hline $\begin{array}{l}\text { 4-methyl- } \\
\text { 1-pentene }\end{array}$ & $7.54 \mathrm{M}$ & 25.0 & ethylene & $0.5 \mathrm{M}$ & - & 1.0 & 75 & 1.0 & 184,000 & 5.000 \\
\hline 1-octene & $6.17 \mathrm{M}$ & 25.0 & ethylene & $0.5 \mathrm{M}$ & - & 0.5 & 75 & 1.0 & 477,060 & 6.480 \\
\hline $\begin{array}{l}\text { 4-methyl- } \\
\text { 1-pentene }\end{array}$ & $3.62 \mathrm{M}$ & 12.0 & 1-octene & $3.19 \mathrm{M}$ & 13.0 & 1.0 & 75 & 1.0 & 3,200 & 0.087 \\
\hline
\end{tabular}

${ }^{\mathrm{a}} 1.63 \mu \mathrm{mol} 2$ dissolved in $1.0 \mathrm{~mL}$ toluene; 1000 equivalents MAO.

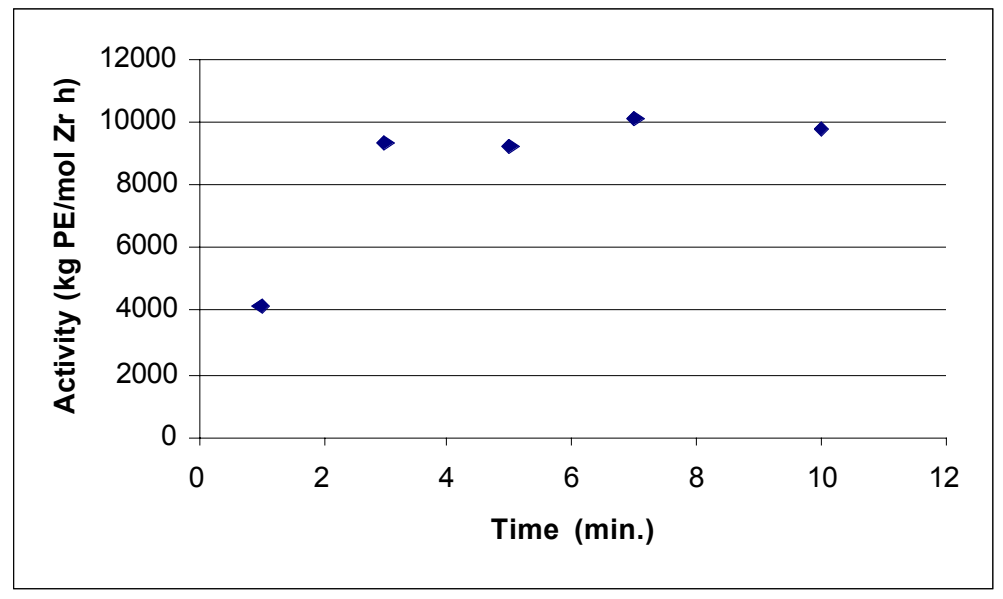

Figure 1. Ethylene homopolymerization activity of $1 / \mathrm{MAO}$ demonstrating that there is an induction period before a nearly constant activity is achieved. Conditions: $1.66 \mu \mathrm{mol} 1$ dissolved in $1.0 \mathrm{~mL}$ toluene; 1000 equivalents $\mathrm{MAO} ; 75^{\circ} \mathrm{C} ; 80$ psi ethylene; 10 minute pre-run equilibration in $25 \mathrm{~mL}$ toluene. 


\section{${ }^{1}$ H NMR Spectra}

\section{2,5-dichloro-2,5-dimethylhexane $\left(\mathrm{C}_{8} \mathrm{H}_{16} \mathrm{Cl}_{2}\right)$}<smiles>CC(C)(Cl)CCC(C)(C)Cl</smiles>

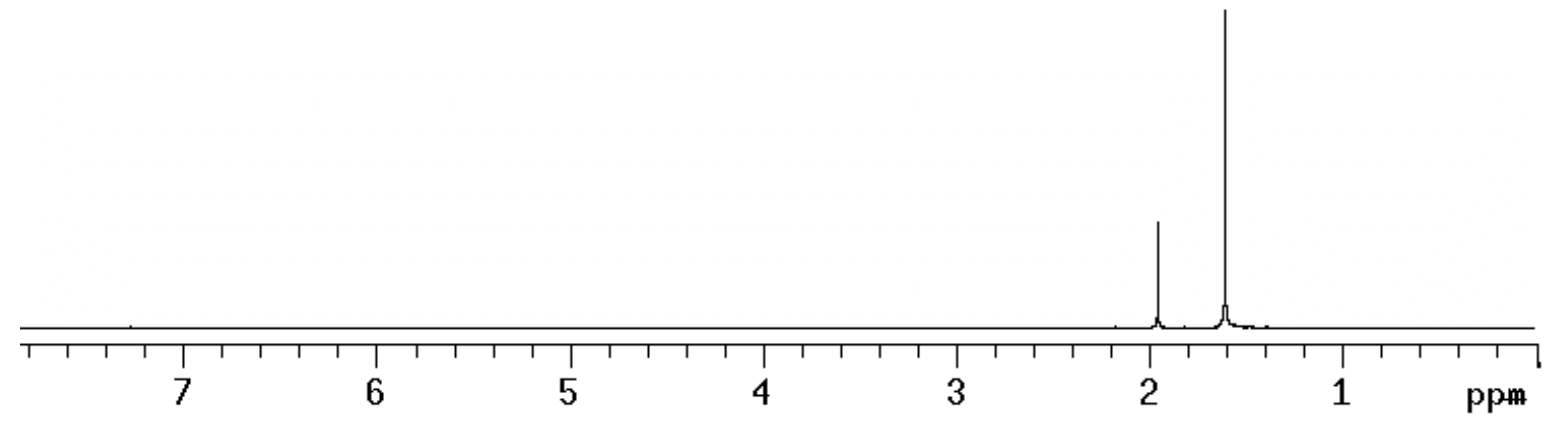

$1,1,4,4,7,7,10,10$-octamethyl-1,2,3,4,7,8,9,10-octahydrodibenzo $[\mathrm{b}, \mathrm{h}]$ fluorene $\left(\mathbf{C}_{29} \mathbf{H}_{38}\right)$
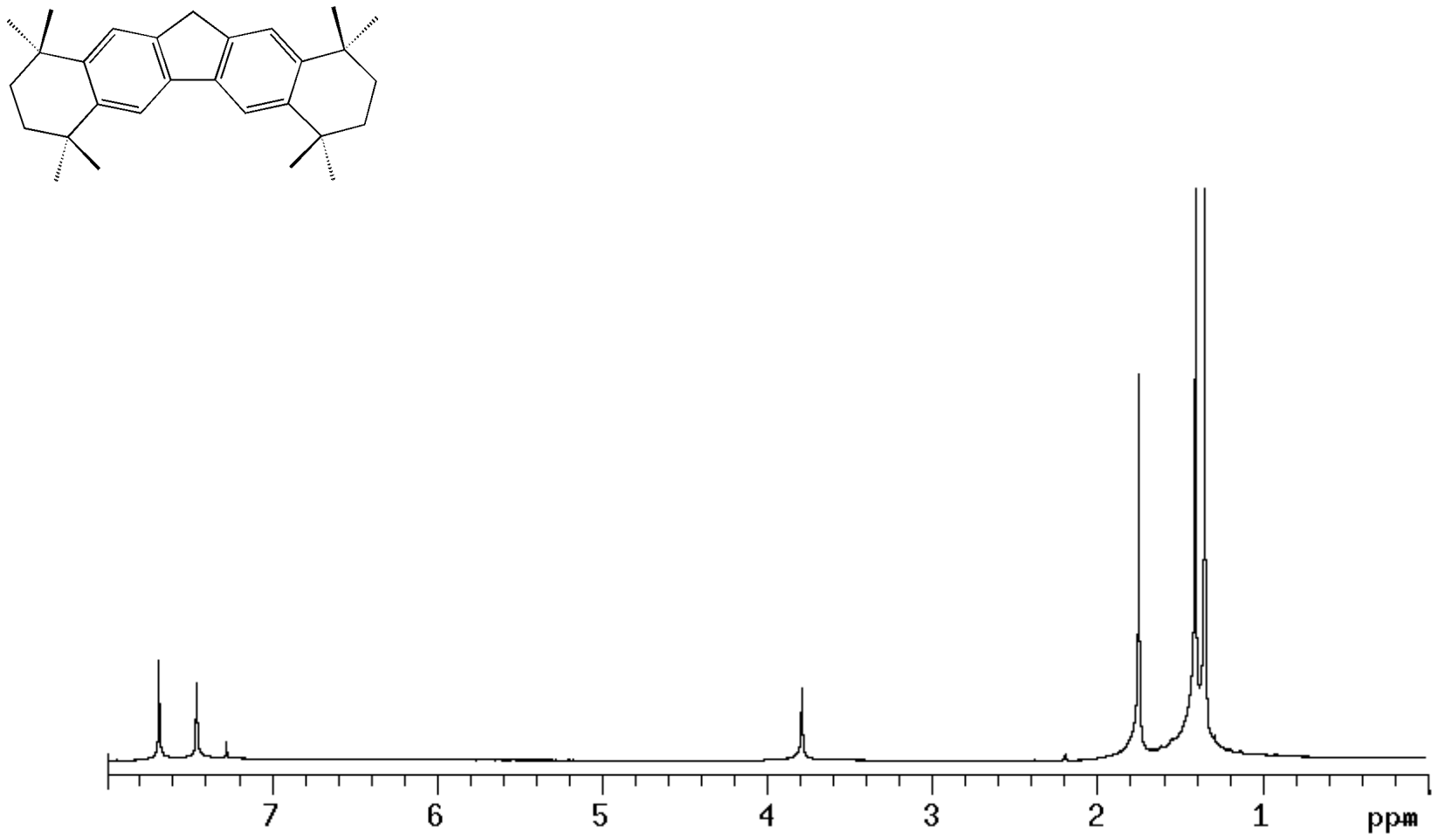
$\operatorname{ClSi}\left(\mathrm{CH}_{3}\right)_{2}\left(\mathrm{C}_{29} \mathrm{H}_{37}\right)$
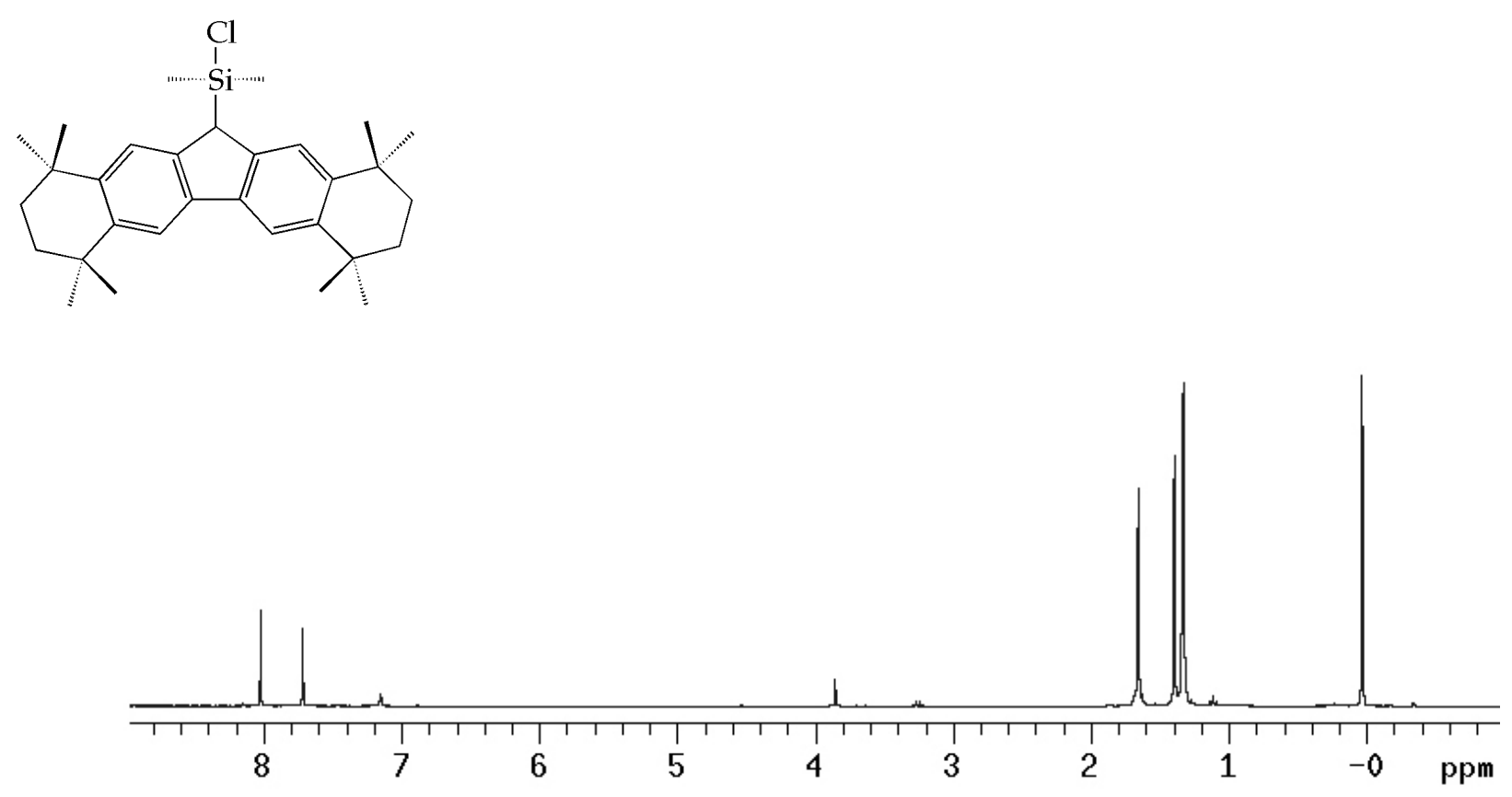

$\left(\mathrm{CH}_{3}\right)_{3} \mathrm{CNH}\left(\mathrm{CH}_{3}\right)_{2} \mathrm{Si}\left(\mathrm{C}_{29} \mathrm{H}_{37}\right)$
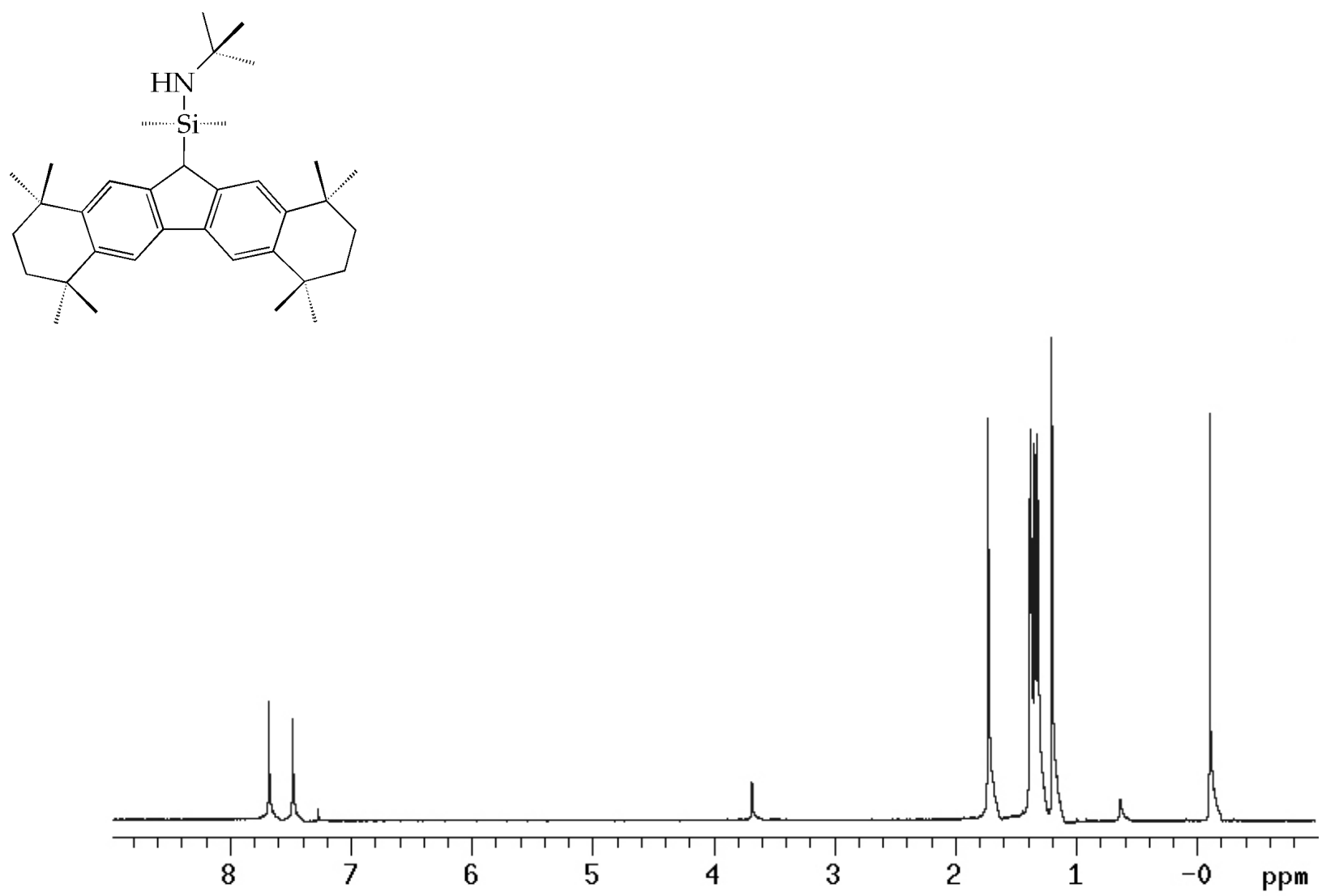
$\mathrm{Me}_{2} \mathrm{Si}\left(\mathrm{C}_{29} \mathrm{H}_{36}\right)\left(\mathrm{N}^{t}{ }^{t} \mathrm{Bu}\right) \mathrm{ZrCl}_{2} \cdot \mathrm{OEt}_{2}$ (1)
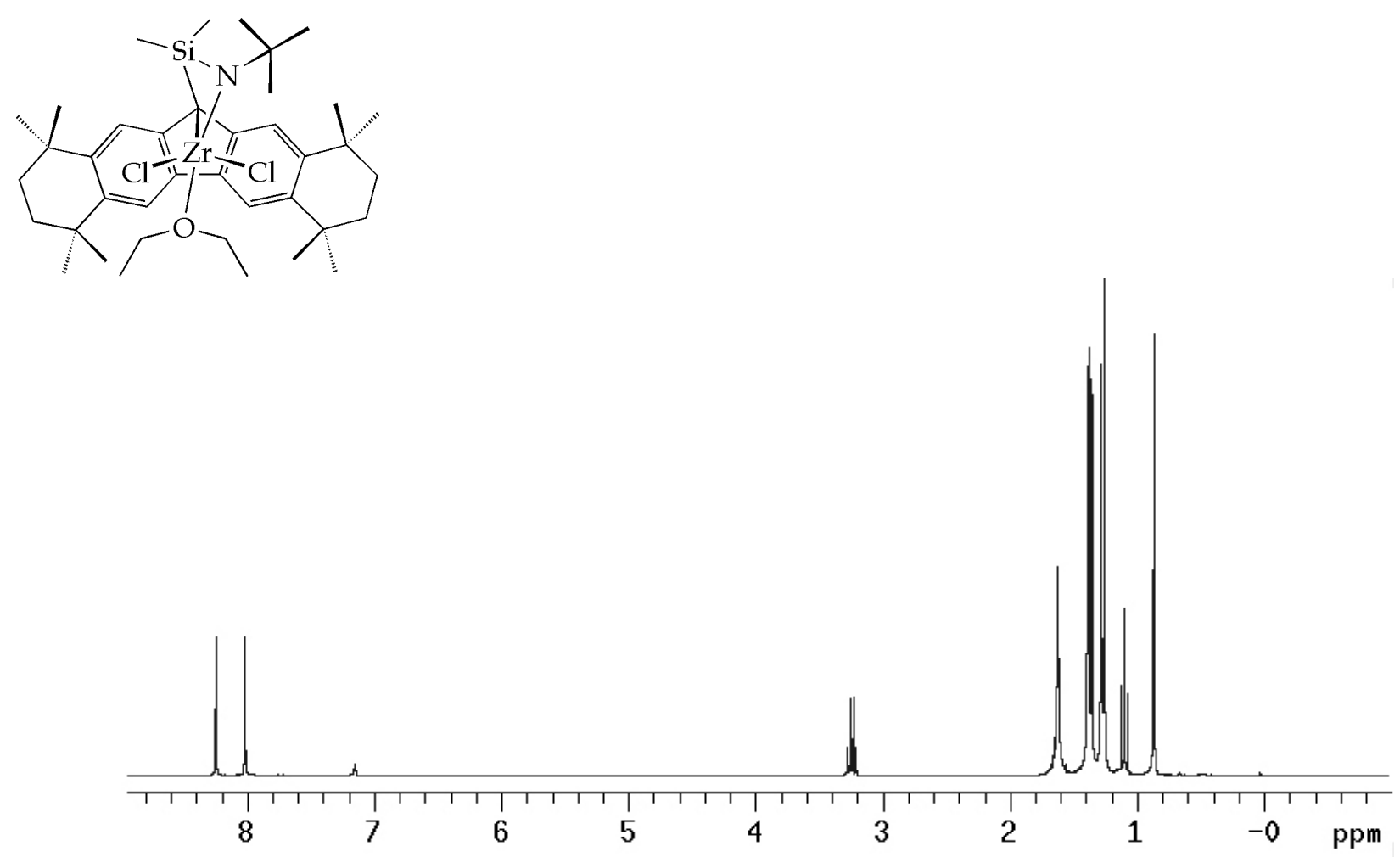
${ }^{13}$ C NMR Spectra

2,5-dichloro-2,5-dimethylhexane $\left(\mathrm{C}_{8} \mathrm{H}_{16} \mathrm{Cl}_{2}\right)$<smiles>CC(C)(Cl)CCC(C)(C)Cl</smiles>

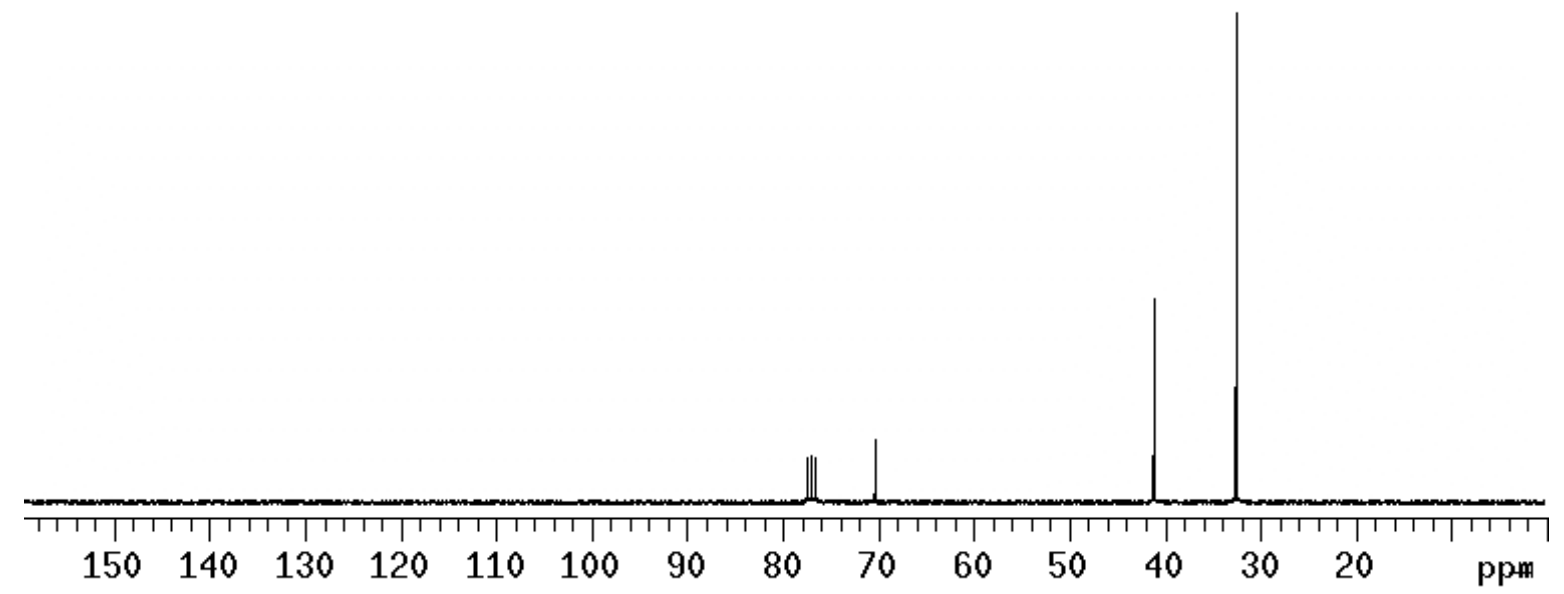

$1,1,4,4,7,7,10,10$-octamethyl-1,2,3,4,7,8,9,10-octahydrodibenzo $[\mathrm{b}, \mathrm{h}]$ fluorene $\left(\mathrm{C}_{29} \mathrm{H}_{38}\right)$
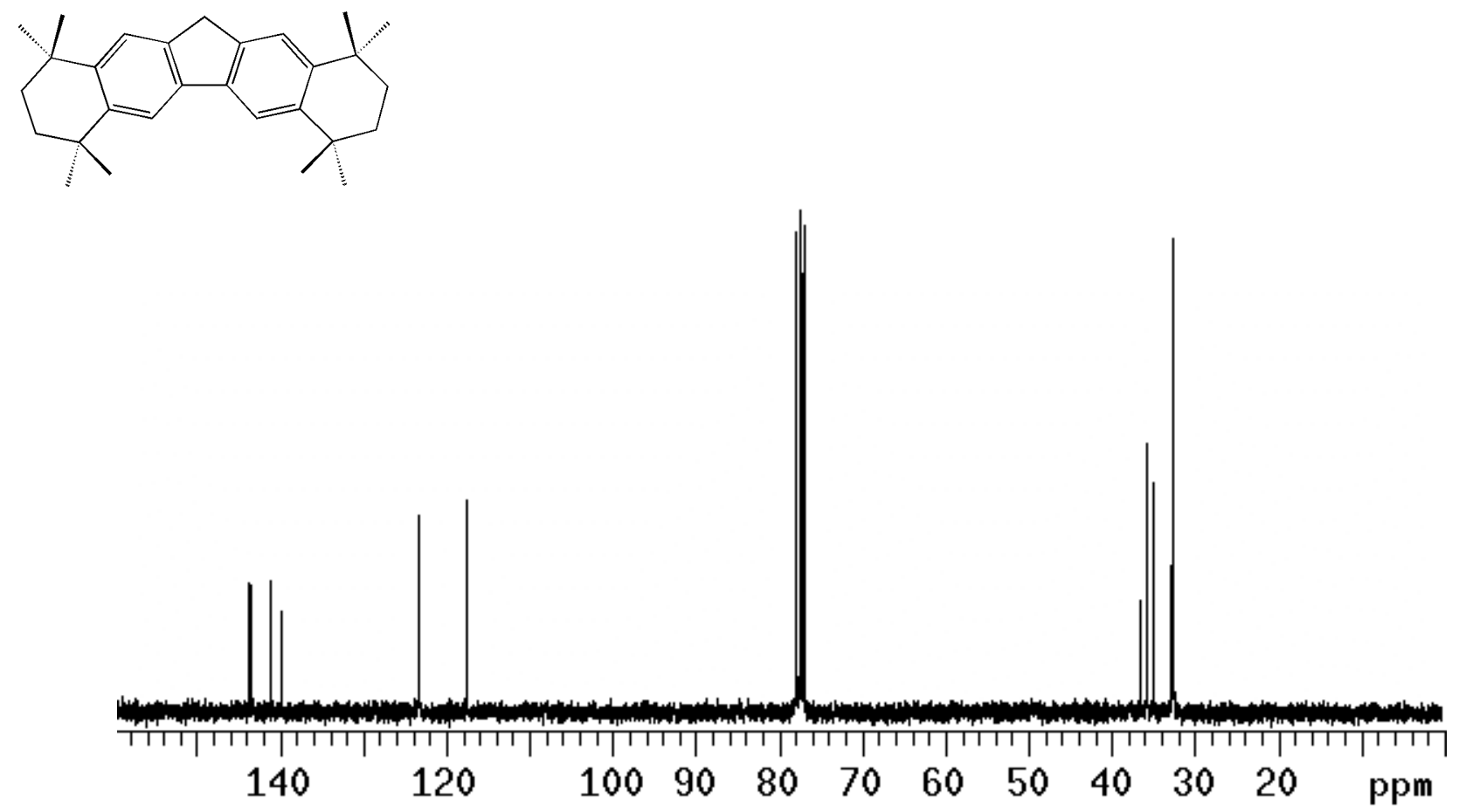
$\operatorname{ClSi}\left(\mathrm{CH}_{3}\right)_{2}\left(\mathrm{C}_{29} \mathrm{H}_{37}\right)$
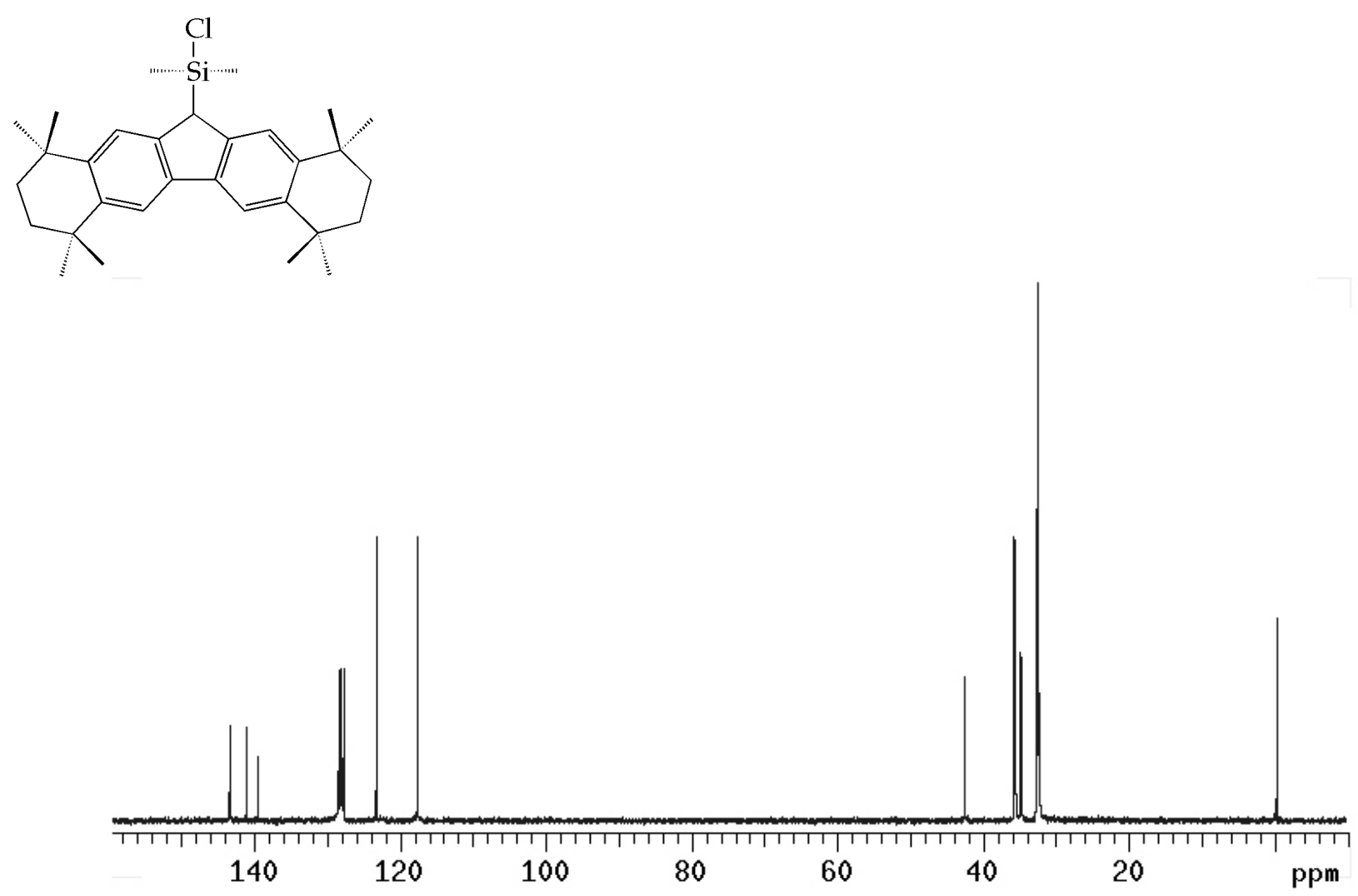

$\left(\mathrm{CH}_{3}\right)_{3} \mathrm{CNH}\left(\mathrm{CH}_{3}\right)_{2} \mathrm{Si}\left(\mathrm{C}_{29} \mathrm{H}_{37}\right)$
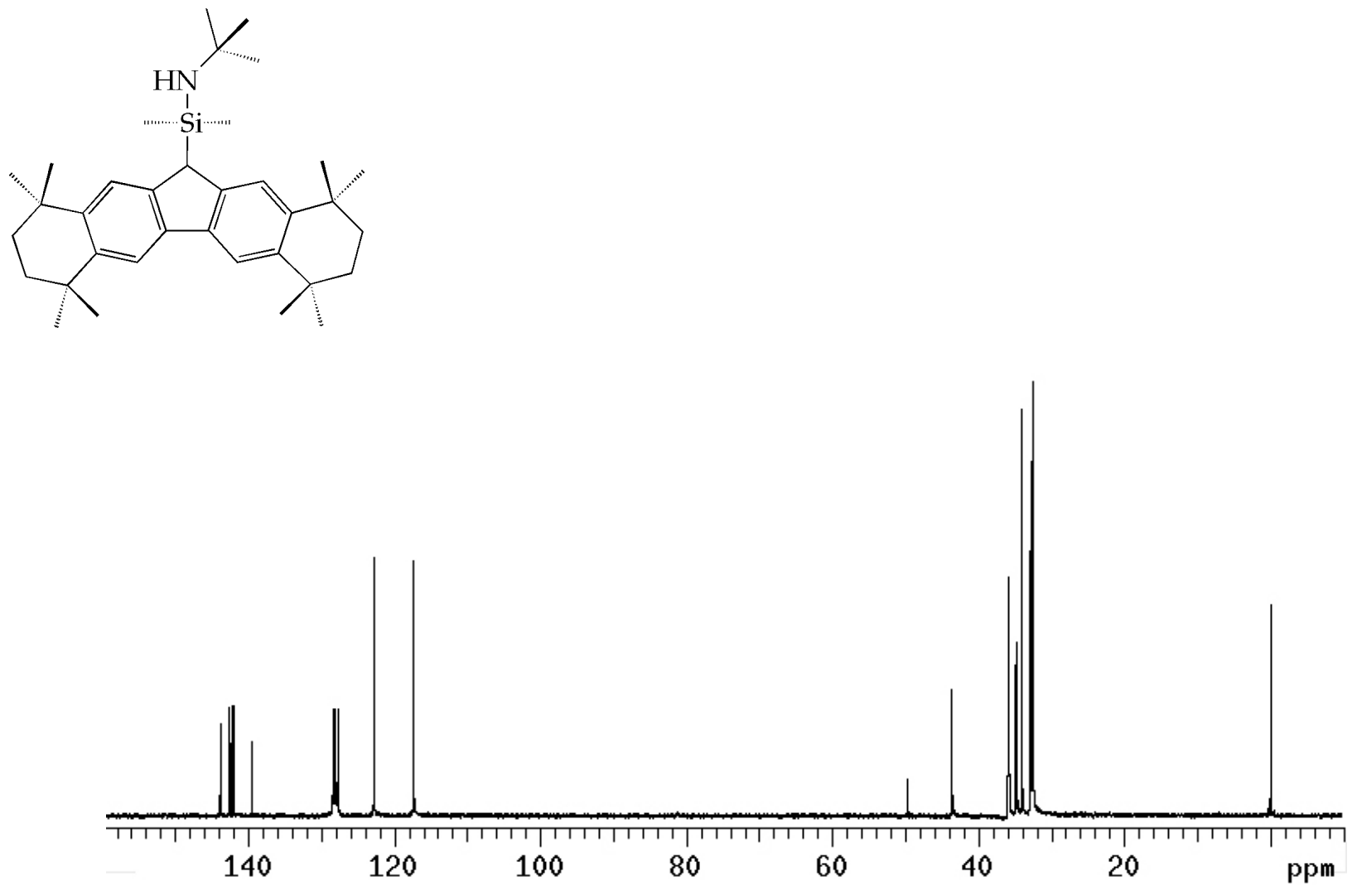
$\mathrm{Me}_{2} \mathrm{Si}\left(\mathrm{C}_{29} \mathrm{H}_{36}\right)\left(\mathrm{N}^{t}{ }^{t} \mathrm{Bu}\right) \mathrm{ZrCl}_{2} \cdot \mathrm{OEt}_{2}$ (1)
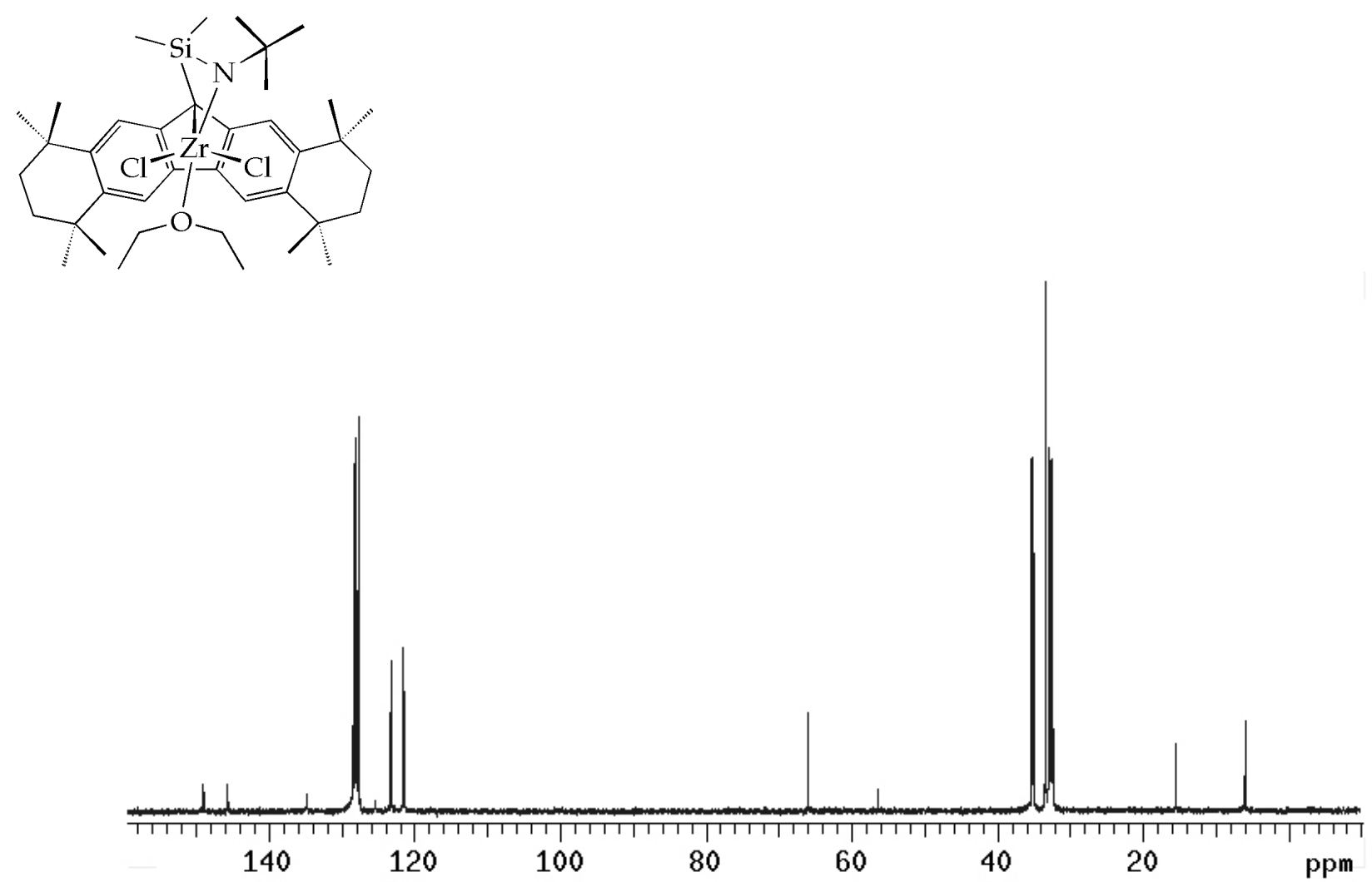


\section{Data Used to Generate Figure 2}

Table 3. Ethylene/4-methyl-1-pentene copolymerizations with 1/MAO. ${ }^{a}$

\begin{tabular}{cccccc}
\hline Entry & {$[\mathbf{P}](\mathbf{M})$} & yield $(\mathrm{g})$ & mol\% $^{\mathrm{b}}$ & activity $^{\mathrm{c}}$ & $\% \mathbf{P}$ feed consumed \\
\hline 1 & 1.0 & 0.60 & 20.8 & 21,690 & 5.7 \\
\hline 2 & 2.0 & 0.86 & 31.1 & 31,120 & 6.1 \\
\hline 3 & 3.0 & 1.33 & 47.0 & 48,070 & 9.5 \\
\hline 4 & 4.0 & 1.99 & 49.8 & 71,960 & 11.3 \\
\hline 5 & 5.0 & 2.89 & 65.7 & 104,460 & 17.3 \\
\hline 6 & 6.0 & 3.98 & 70.5 & 143,860 & 21.4 \\
\hline 7 & 7.54 & 5.79 & $>95$ & 209,280 & 33.3 \\
\hline
\end{tabular}

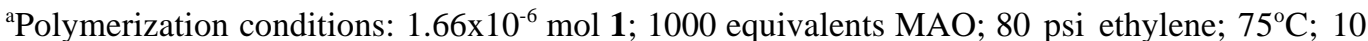
min. pre-run equilibration; 1 min. run. ${ }^{b} \mathbf{P}=4$-methyl-1-pentene, mol\% incorporation determined by ${ }^{13} \mathrm{C}$ NMR according to the method of Kimura et al. ${ }^{2}{ }^{\mathrm{c}}$ Activity expressed in $\mathrm{kg} /(\mathrm{mol} \mathrm{Zr} \cdot \mathrm{h})$ and rounded to the nearest $10^{1}$. ${ }^{\mathrm{d}}$ This number represents the fraction of the initial amount of 4methyl-1-pentene that is converted into polymer. 
${ }^{13}$ C NMR of ethylene/4-methyl-1-pentene Copolymers

Table 3, Entry 1: 20.8 mol\% 4-methyl-1-pentene incorporation

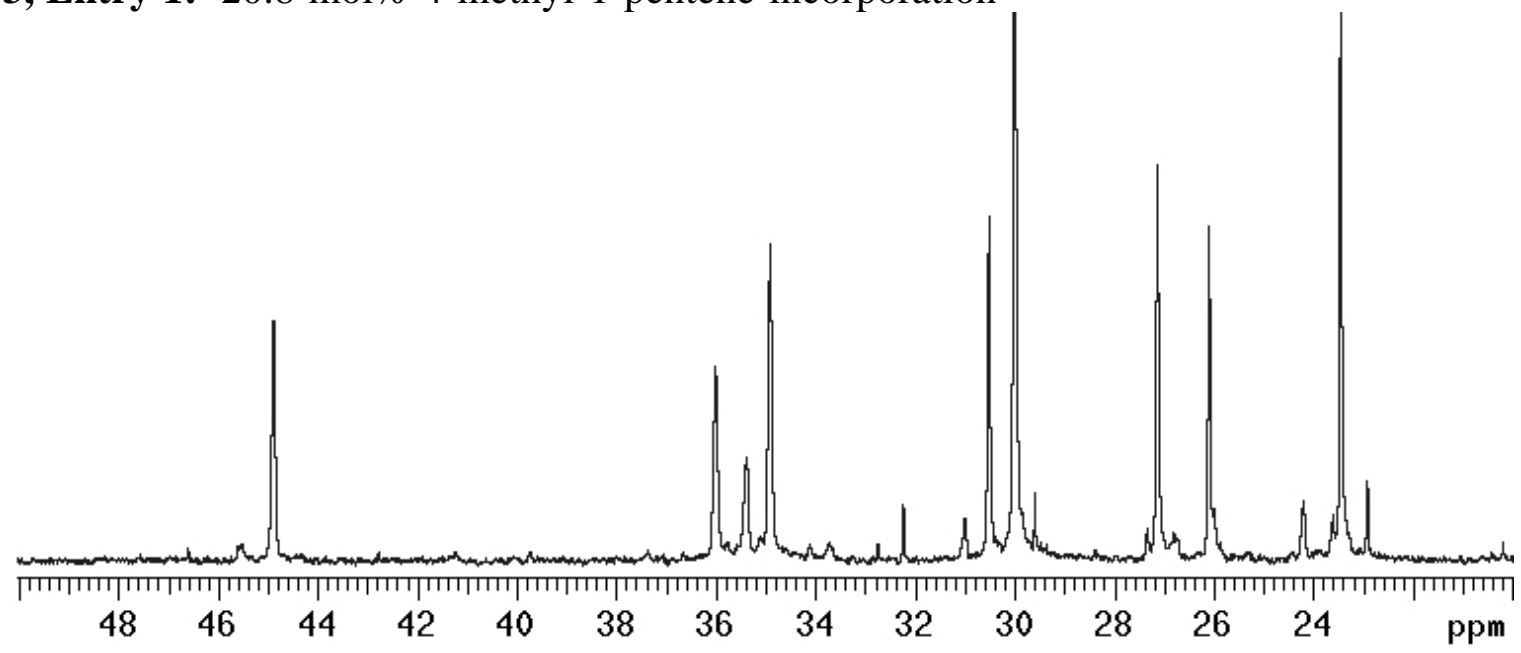

Table 3, Entry 2: 31.1 mol\% 4-methyl-1-pentene incorporation

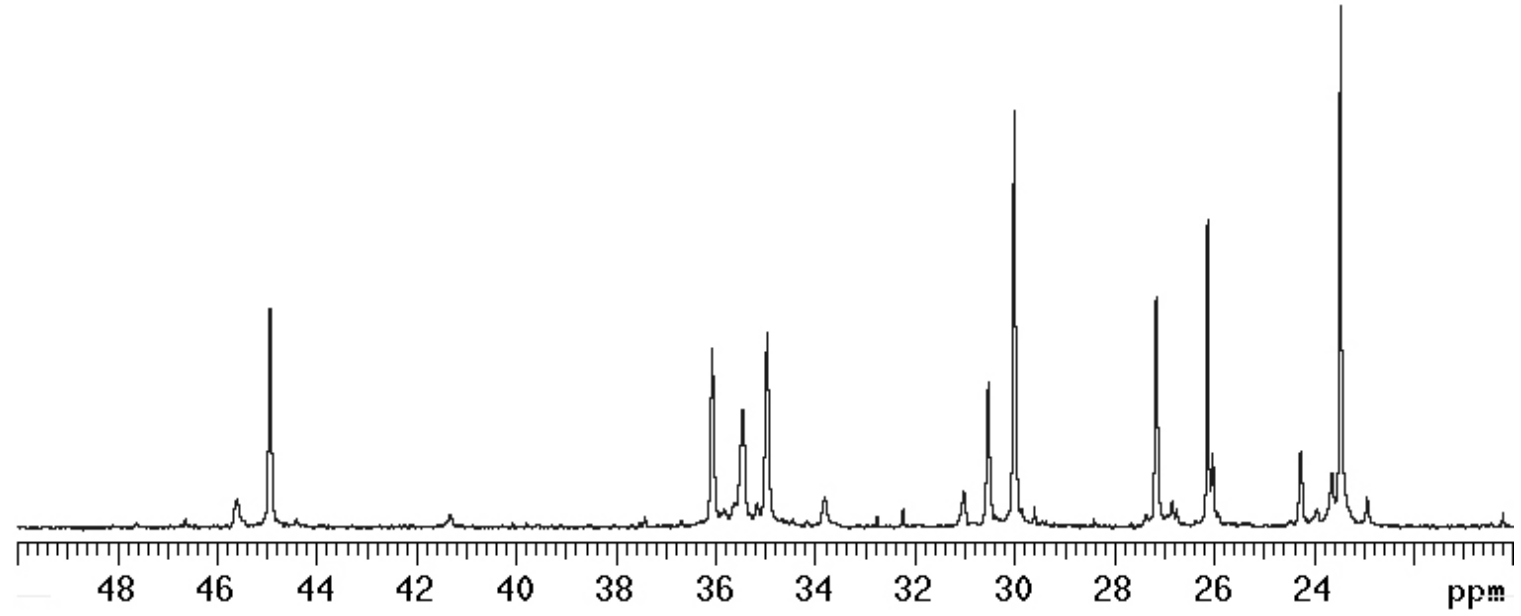

Table 3, Entry 3: 47.0 mol\% 4-methyl-1-pentene incorporation

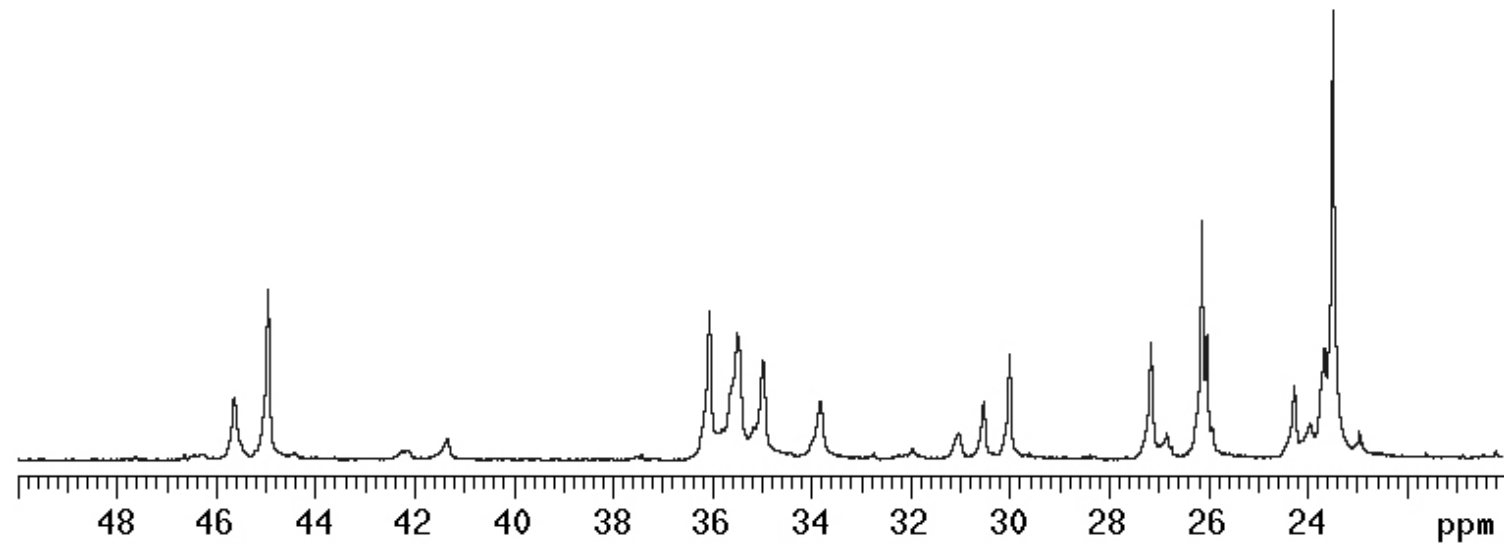


Table 3, Entry 4: 49.8 mol\% 4-methyl-1-pentene incorporation

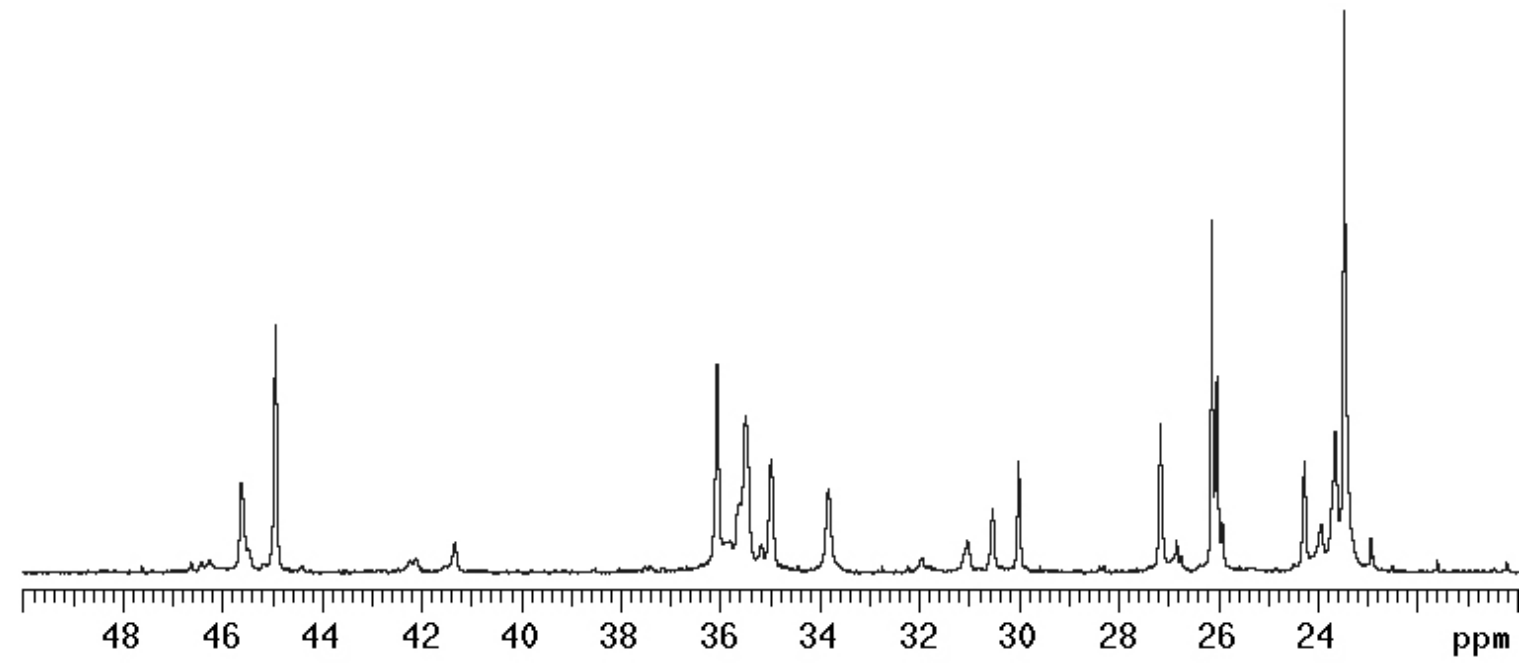

Table 3, Entry 5: 65.7 mol\% 4-methyl-1-pentene incorporation

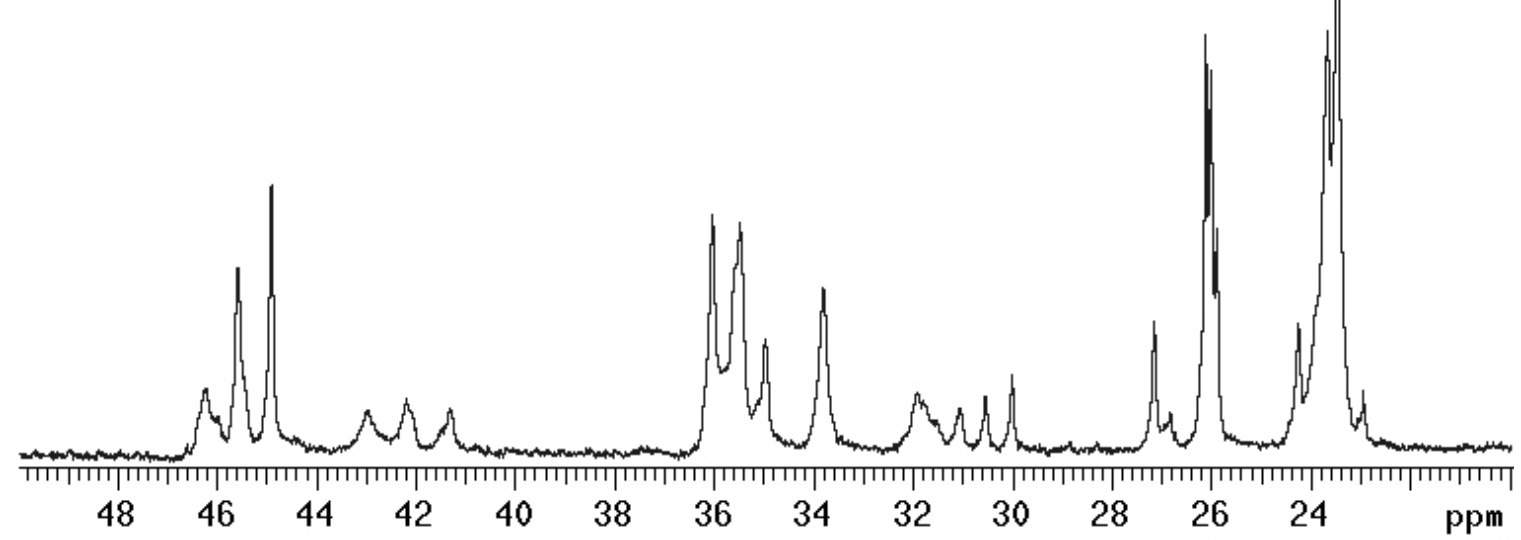

Table 3, Entry 6: 70.5 mol\% 4-methyl-1-pentene incorporation

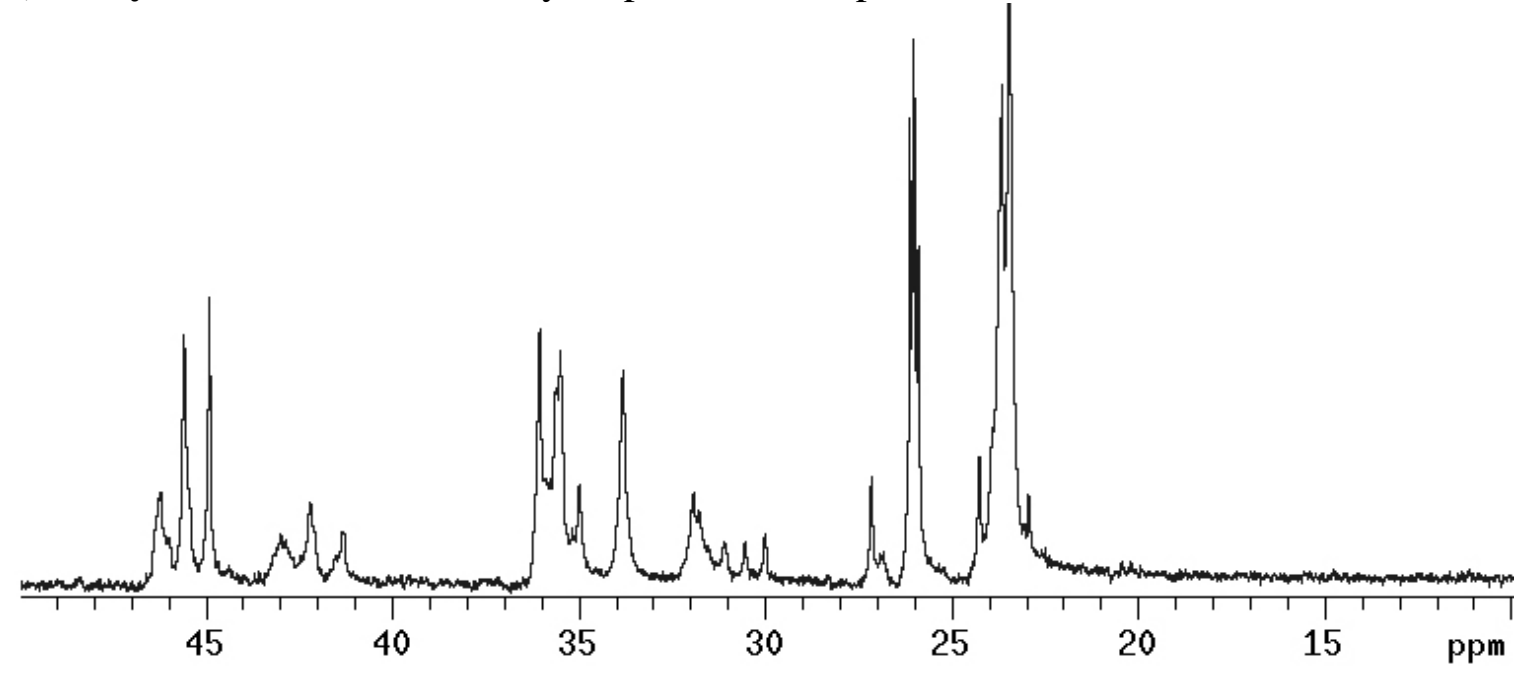


Table 3, Entry 7: >95 mol\% 4-methyl-1-pentene incorporation

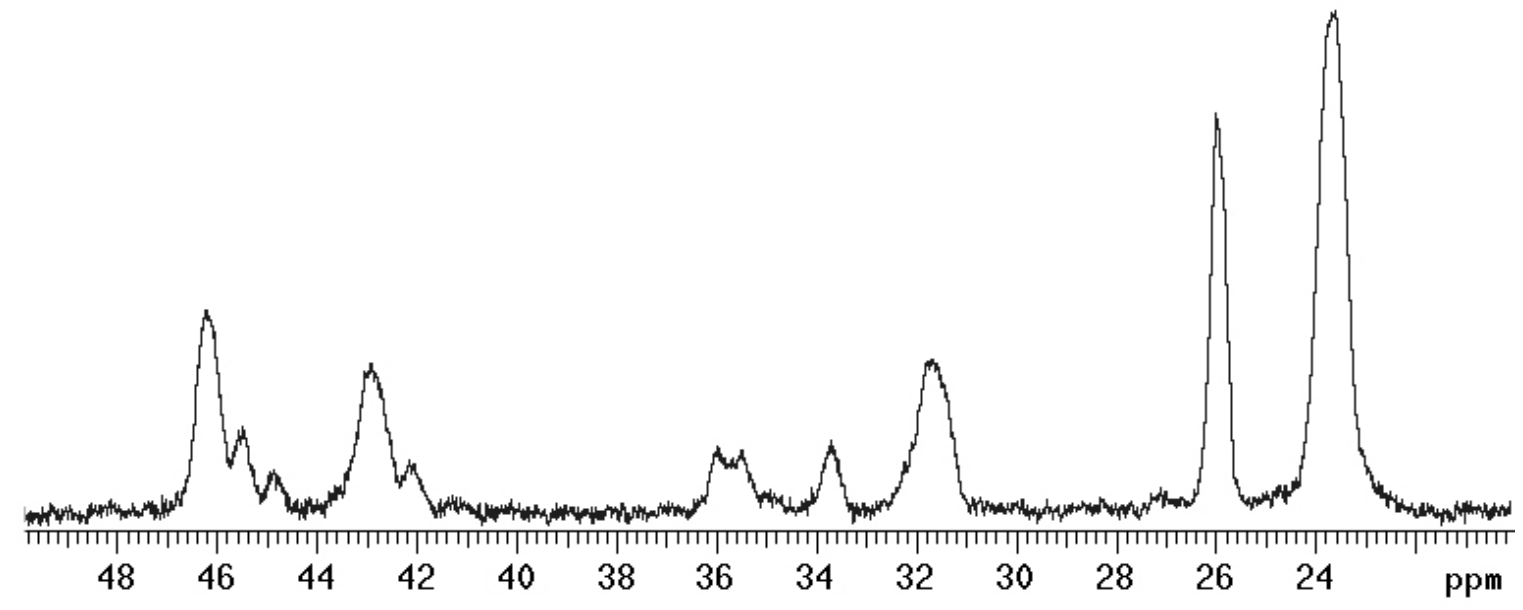




\section{Data Used to Generate Figure 3}

Table 4. Ethylene/1-octene copolymerizations with 1/MAO. ${ }^{a}$

\begin{tabular}{cccccc}
\hline Entry & {$[\mathbf{O}](\mathrm{M})$} & yield $(\mathrm{g})$ & $\mathrm{mol}_{\mathbf{0}} \mathbf{O}^{\mathrm{b}}$ & activity $^{\mathrm{c}}$ & $\% \mathbf{O}$ feed consumed \\
\hline 1 & 0.98 & 0.934 & 32.3 & 33,760 & 10.5 \\
\hline 2 & 1.96 & 2.37 & 48.5 & 85,660 & 20.1 \\
\hline 3 & 2.94 & 4.37 & 53.8 & 157,950 & 27.4 \\
\hline 4 & 3.92 & 6.68 & 66.1 & 241,450 & 38.6 \\
\hline 5 & 4.90 & 8.41 & 69.1 & 303,980 & 40.6 \\
\hline 6 & 6.13 & 12.11 & 74.8 & 437,710 & 50.7 \\
\hline
\end{tabular}

${ }^{\text {a} P o l y m e r i z a t i o n ~ c o n d i t i o n s: ~} 1.66 \times 10^{-6}$ mol 1; 1000 equivalents $\mathrm{MAO} ; 80$ psi ethylene; $75^{\circ} \mathrm{C} ; 10$ min. pre-run equilibration; $1 \mathrm{~min}$. run. ${ }^{\mathrm{b}} \mathbf{O}=1$-octene, mol\% incorporation determined by ${ }^{13} \mathrm{C}$ NMR according to the method of Kimura et al. ${ }^{2}{ }^{\mathrm{c}}$ Activity expressed in $\mathrm{kg}(\mathrm{mol} \mathrm{Zr} \cdot \mathrm{h})^{-1}$ and rounded to the nearest $10^{1}$. ${ }^{\mathrm{d}}$ This number represents the fraction of the initial amount of 1 -octene that is converted into polymer. 
${ }^{13}$ C NMR of ethylene/1-octene Copolymers

Table 4, Entry 1: $32.3 \%$ 1-octene incorporation

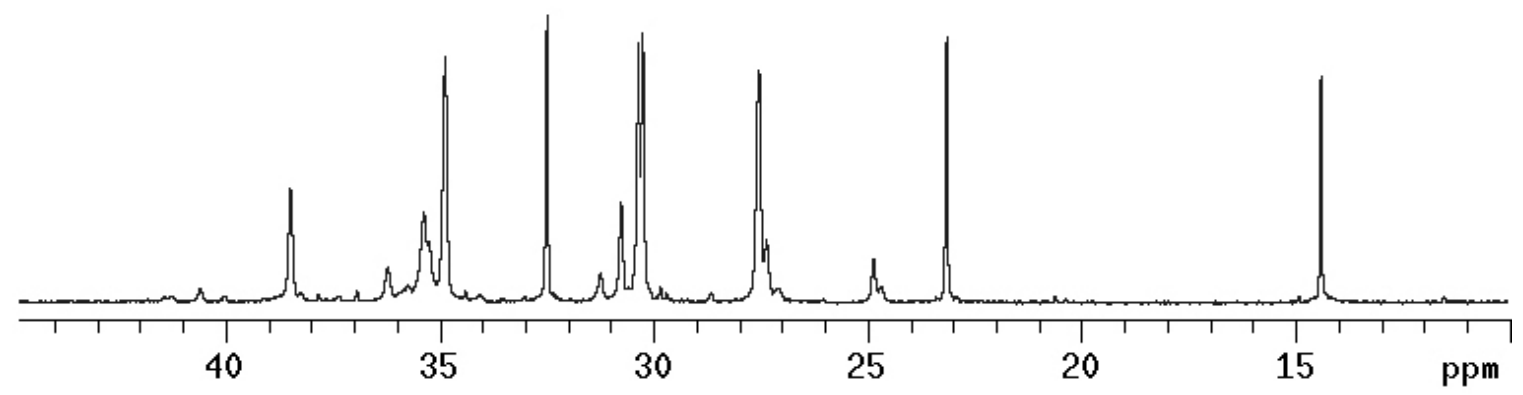

Table 4, Entry 2: $48.5 \%$ 1-octene incorporation

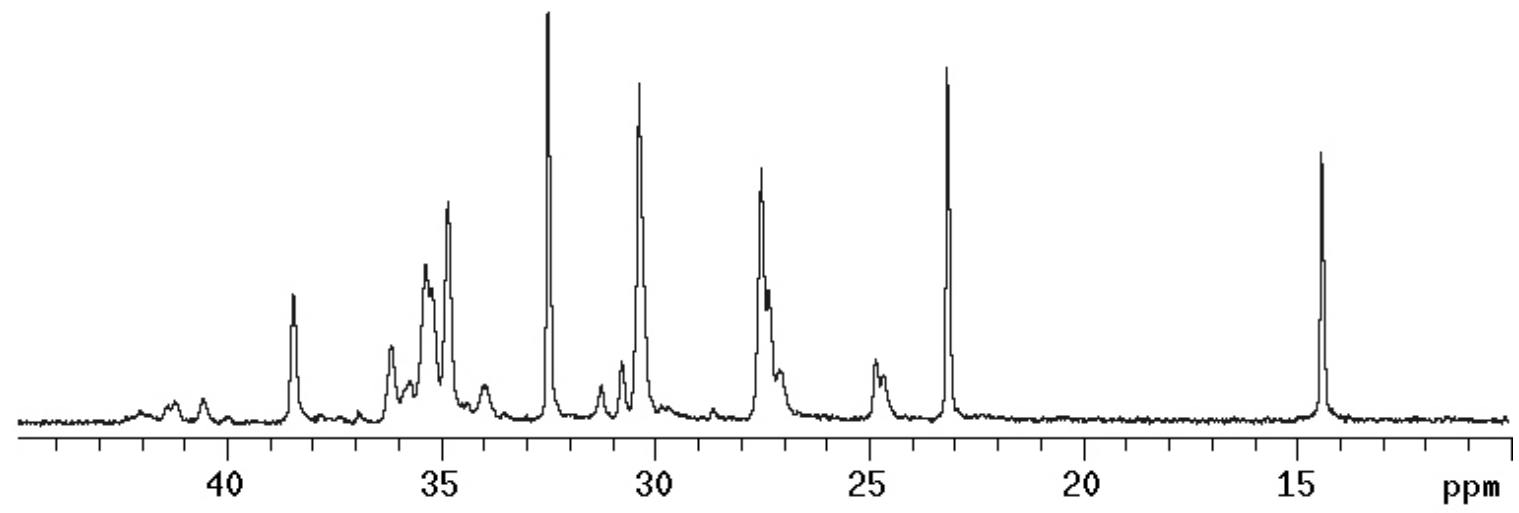

Table 4, Entry 3: 53.8\% 1-octene incorporation

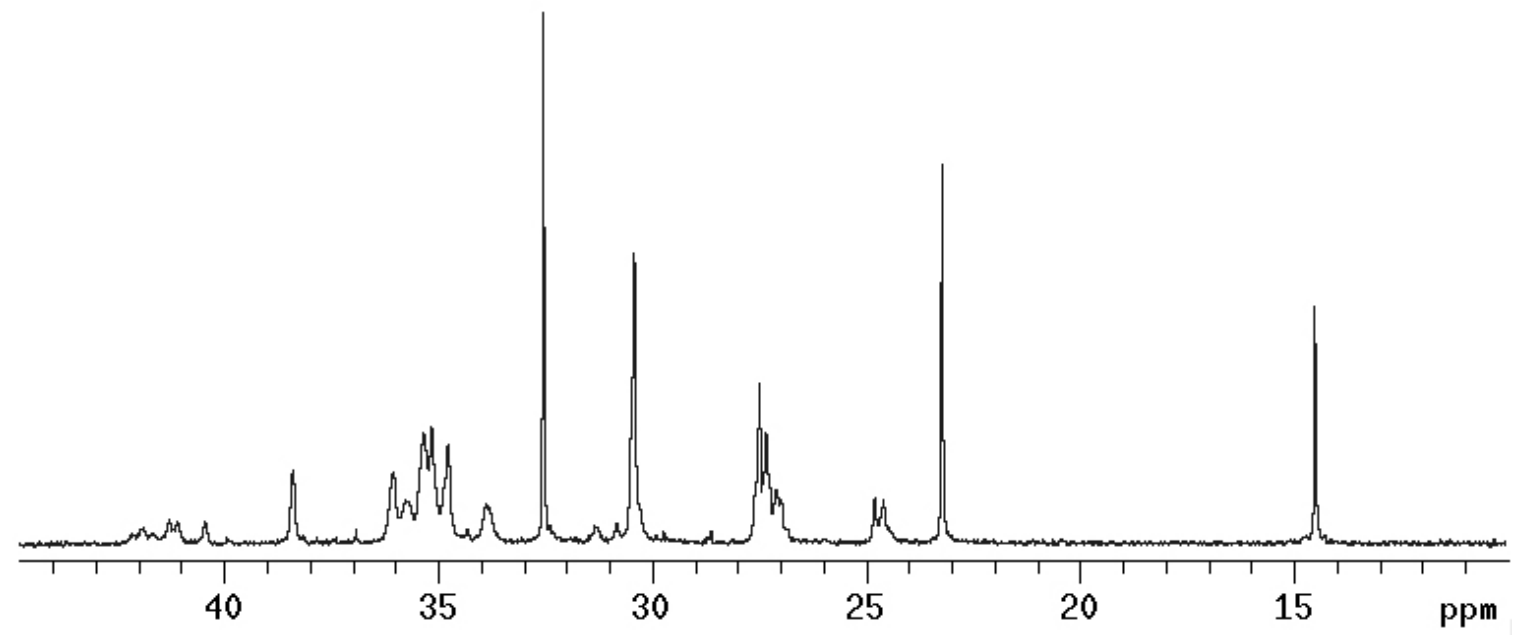


Table 4, Entry 4: $66.1 \%$ 1-octene incorporation

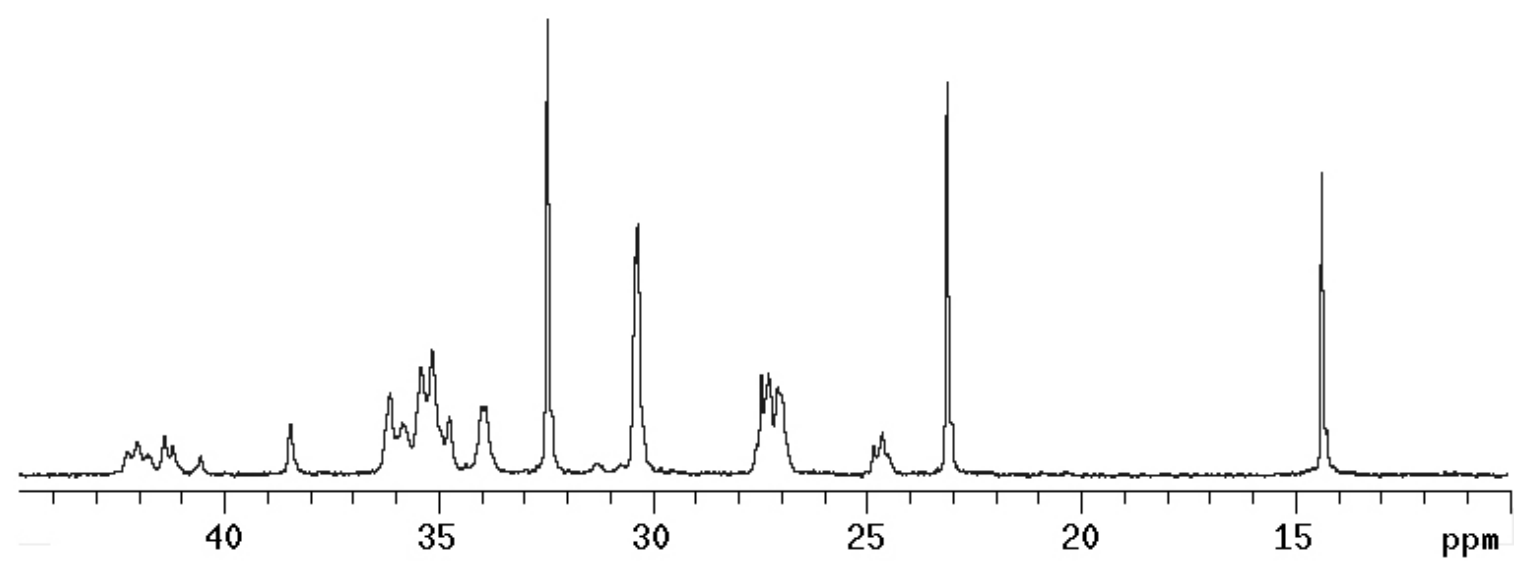

Table 4, Entry 5: 69.1\% 1-octene incorporation

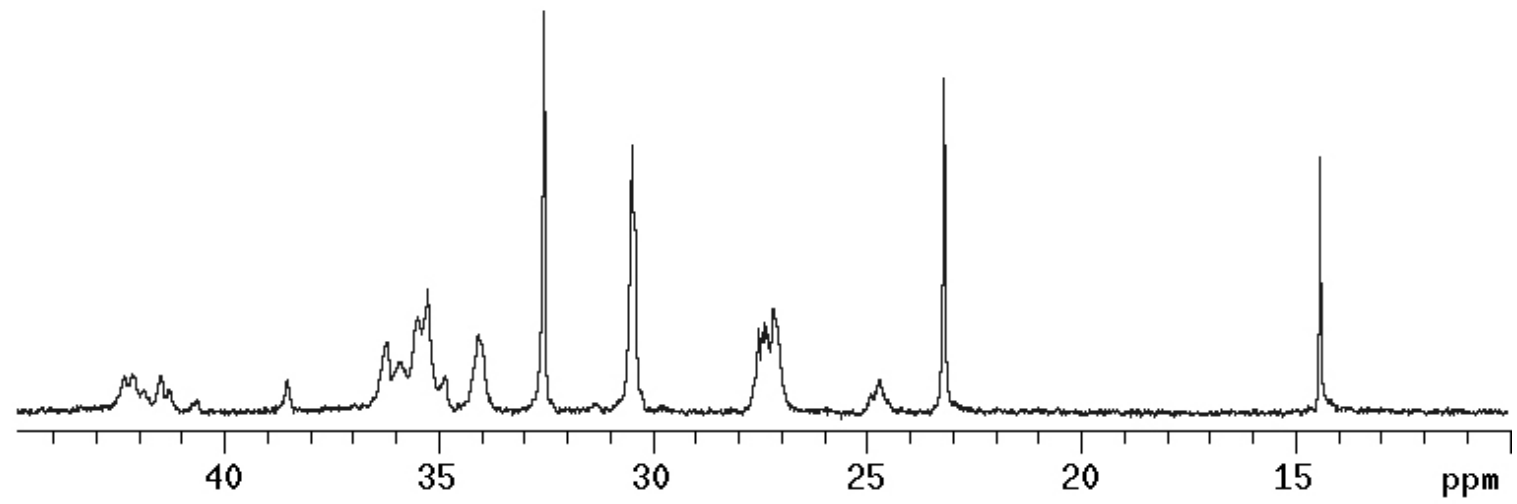

Table 4, Entry 6: $74.8 \%$ 1-octene incorporation

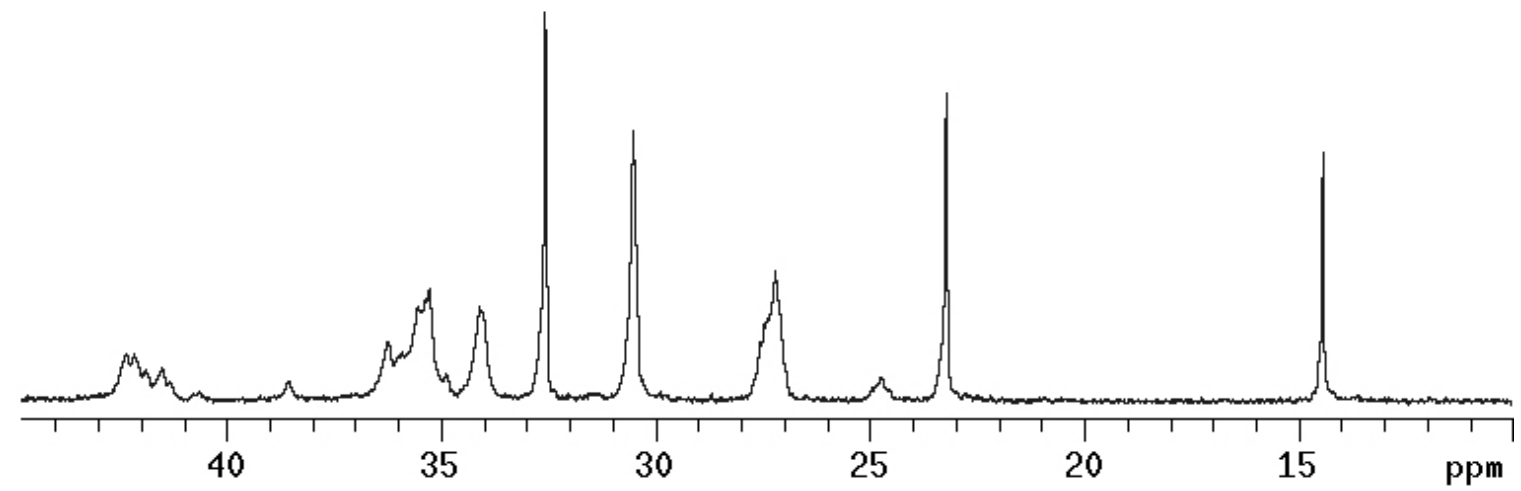




\section{X-Ray Structure Data for $1,1,4,4,7,7,10,10$-octamethyl-1,2,3,4,7,8,9,10- octahydrodibenzo $[\mathrm{b}, \mathrm{h}]$ fluorene (sm34)

$$
\mathrm{C}_{29} \mathbf{H}_{38}
$$

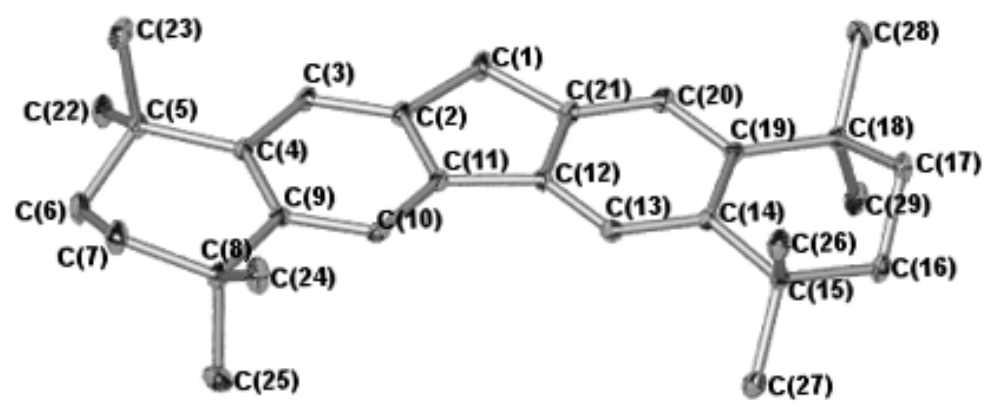

Labeled view with $50 \%$ probability ellipsoids

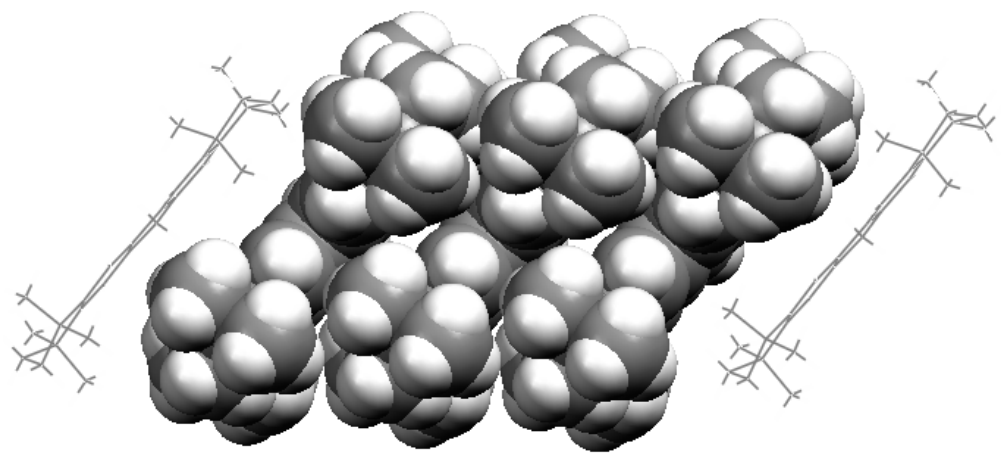

A wire frame and space filling view of $\mathrm{C}_{29} \mathrm{H}_{38}$ illustrating how the methyl groups of adjacent molecules pack tightly into the fluorenyl-based cavity

Table 5. Crystal structure and data refinement (sm34): $\mathrm{C}_{29} \mathrm{H}_{38}$

\section{Identification code \\ Empirical formula \\ Formula weight \\ Temperature \\ Wavelength \\ Crystal system \\ Space group \\ Unit cell dimensions}

Volume

Z

Density (calculated)

Absorption coefficient

$\mathrm{F}(000)$

Crystal size

Theta range for data collection

Index ranges

Reflections collected

Independent reflections

Completeness to theta $=28.29^{\circ}$

Absorption correction

Max. and min. transmission

Refinement method

Data / restraints / parameters

Goodness-of-fit on $\mathrm{F}^{2}$

\section{$\operatorname{sm} 34$}

C29 H38

386.59

$110(2) \mathrm{K}$

$0.71073 \AA$

Monoclinic

$\mathrm{P} 2(1) / \mathrm{c}$

$$
\begin{array}{ll}
\mathrm{a}=5.7601(15) \AA & \alpha=90^{\circ} . \\
\mathrm{b}=16.182(4) \AA & \beta=92.950(4)^{\circ} . \\
\mathrm{c}=24.356(6) \AA & \gamma=90^{\circ} .
\end{array}
$$

2267.2(10) $\AA^{3}$

4

$1.133 \mathrm{Mg} / \mathrm{m}^{3}$

$0.063 \mathrm{~mm}^{-1}$

848

$0.60 \times 0.40 \times 0.20 \mathrm{~mm}^{3}$

1.51 to $28.29^{\circ}$.

$-7<=\mathrm{h}<=7,-21<=\mathrm{k}<=20,-31<=1<=32$

22289

$5515[\mathrm{R}(\mathrm{int})=0.0579]$

$97.7 \%$

Semi-empirical from equivalents 0.9875 and 0.9632

Full-matrix least-squares on $\mathrm{F}^{2}$

$5515 / 0 / 270$

1.058 
Final $\mathrm{R}$ indices [I>2sigma(I)]

$\mathrm{R}$ indices (all data)

Largest diff. peak and hole
$\mathrm{R} 1=0.0654, \mathrm{wR} 2=0.1479$

$\mathrm{R} 1=0.0898, \mathrm{wR} 2=0.1588$

0.375 and -0.299 e. $\AA^{-3}$

Table 6. Atomic coordinates $\left(\mathrm{x} 10^{4}\right)$ and equivalent isotropic displacement parameters $\left(\AA^{2} \times 10^{3}\right)$ for (sm34). U(eq) is defined as one third of the trace of the orthogonalized $\mathrm{U}^{\mathrm{ij}}$ tensor.

\begin{tabular}{lrrrr}
\hline & $\mathrm{x}$ & $\mathrm{y}$ & $\mathrm{z}$ & $\mathrm{U}(\mathrm{eq})$ \\
\hline $\mathrm{C}(1)$ & $7617(4)$ & $4428(1)$ & $4582(1)$ & $18(1)$ \\
$\mathrm{C}(2)$ & $6243(3)$ & $3630(1)$ & $4567(1)$ & $14(1)$ \\
$\mathrm{C}(3)$ & $4883(3)$ & $3286(1)$ & $4957(1)$ & $14(1)$ \\
$\mathrm{C}(4)$ & $3798(3)$ & $2515(1)$ & $4869(1)$ & $12(1)$ \\
$\mathrm{C}(5)$ & $2423(3)$ & $2141(1)$ & $5331(1)$ & $14(1)$ \\
$\mathrm{C}(6)$ & $740(4)$ & $1478(1)$ & $5094(1)$ & $19(1)$ \\
$\mathrm{C}(7)$ & $1975(4)$ & $856(1)$ & $4739(1)$ & $20(1)$ \\
$\mathrm{C}(8)$ & $2973(3)$ & $1256(1)$ & $4231(1)$ & $14(1)$ \\
$\mathrm{C}(9)$ & $4102(3)$ & $2091(1)$ & $4372(1)$ & $11(1)$ \\
$\mathrm{C}(10)$ & $5459(3)$ & $2456(1)$ & $3975(1)$ & $12(1)$ \\
$\mathrm{C}(11)$ & $6548(3)$ & $3210(1)$ & $4073(1)$ & $12(1)$ \\
$\mathrm{C}(12)$ & $8122(3)$ & $3698(1)$ & $3744(1)$ & $12(1)$ \\
$\mathrm{C}(13)$ & $8988(3)$ & $3552(1)$ & $3232(1)$ & $13(1)$ \\
$\mathrm{C}(14)$ & $10460(3)$ & $4128(1)$ & $2993(1)$ & $12(1)$ \\
$\mathrm{C}(15)$ & $11329(3)$ & $3913(1)$ & $2425(1)$ & $13(1)$ \\
$\mathrm{C}(16)$ & $12663(3)$ & $4641(1)$ & $2182(1)$ & $17(1)$ \\
$\mathrm{C}(17)$ & $14124(3)$ & $5120(1)$ & $2609(1)$ & $17(1)$ \\
$\mathrm{C}(18)$ & $12622(3)$ & $5519(1)$ & $3040(1)$ & $13(1)$ \\
$\mathrm{C}(19)$ & $11088(3)$ & $4852(1)$ & $3286(1)$ & $12(1)$ \\
$\mathrm{C}(20)$ & $10221(3)$ & $4983(1)$ & $3808(1)$ & $15(1)$ \\
$\mathrm{C}(21)$ & $8759(3)$ & $4417(1)$ & $4035(1)$ & $14(1)$ \\
$\mathrm{C}(22)$ & $952(3)$ & $2794(1)$ & $5613(1)$ & $19(1)$ \\
$\mathrm{C}(23)$ & $4162(4)$ & $1769(1)$ & $5761(1)$ & $21(1)$ \\
$\mathrm{C}(24)$ & $4734(4)$ & $647(1)$ & $4008(1)$ & $20(1)$ \\
$\mathrm{C}(25)$ & $1022(4)$ & $1397(1)$ & $3787(1)$ & $22(1)$ \\
$\mathrm{C}(26)$ & $12968(3)$ & $3158(1)$ & $2487(1)$ & $18(1)$ \\
$\mathrm{C}(27)$ & $9290(3)$ & $3706(1)$ & $2018(1)$ & $19(1)$ \\
$\mathrm{C}(28)$ & $14264(4)$ & $5927(1)$ & $3476(1)$ & $21(1)$ \\
$\mathrm{C}(29)$ & $11037(4)$ & $6195(1)$ & $2779(1)$ & $20(1)$ \\
\hline
\end{tabular}

Table 7. Bond lengths $[\AA]$ and angles $\left[{ }^{\circ}\right]$ for sm34.

\begin{tabular}{llcc}
\hline $\mathrm{C}(1)-\mathrm{C}(2)$ & $1.515(2)$ & $\mathrm{C}(12)-\mathrm{C}(21)$ & $1.401(2)$ \\
$\mathrm{C}(1)-\mathrm{C}(21)$ & $1.515(2)$ & $\mathrm{C}(13)-\mathrm{C}(14)$ & $1.407(2)$ \\
$\mathrm{C}(2)-\mathrm{C}(3)$ & $1.379(2)$ & $\mathrm{C}(14)-\mathrm{C}(19)$ & $1.410(2)$ \\
$\mathrm{C}(2)-\mathrm{C}(11)$ & $\mathrm{C}(14)-\mathrm{C}(15)$ & $1.535(2)$ \\
$\mathrm{C}(3)-\mathrm{C}(4)$ & $1.401(2)$ & $\mathrm{C}(15)-\mathrm{C}(27)$ & $1.533(3)$ \\
$\mathrm{C}(4)-\mathrm{C}(9)$ & $1.406(2)$ & $\mathrm{C}(15)-\mathrm{C}(16)$ & $1.541(2)$ \\
$\mathrm{C}(4)-\mathrm{C}(5)$ & $1.411(2)$ & $\mathrm{C}(15)-\mathrm{C}(26)$ & $1.546(3)$ \\
$\mathrm{C}(5)-\mathrm{C}(23)$ & $1.534(2)$ & $\mathrm{C}(16)-\mathrm{C}(17)$ & $1.517(3)$ \\
$\mathrm{C}(5)-\mathrm{C}(22)$ & $1.535(3)$ & $\mathrm{C}(17)-\mathrm{C}(18)$ & $1.536(2)$ \\
$\mathrm{C}(5)-\mathrm{C}(6)$ & $1.537(2)$ & $\mathrm{C}(18)-\mathrm{C}(28)$ & $1.535(3)$ \\
$\mathrm{C}(6)-\mathrm{C}(7)$ & $1.539(3)$ & $\mathrm{C}(18)-\mathrm{C}(19)$ & $1.535(2)$ \\
$\mathrm{C}(7)-\mathrm{C}(8)$ & $1.526(3)$ & $\mathrm{C}(18)-\mathrm{C}(29)$ & $1.541(3)$ \\
& $1.535(2)$ & $\mathrm{C}(19)-\mathrm{C}(20)$ & $1.406(2)$ \\
$\mathrm{C}(8)-\mathrm{C}(9)$ & & $\mathrm{C}(20)-\mathrm{C}(21)$ & $1.379(2)$ \\
$\mathrm{C}(8)-\mathrm{C}(24)$ & $1.530(2)$ & $\mathrm{C}(2)-\mathrm{C}(1)-\mathrm{C}(21)$ & $102.64(14)$ \\
$\mathrm{C}(8)-\mathrm{C}(25)$ & $1.535(2)$ & $\mathrm{C}(3)-\mathrm{C}(2)-\mathrm{C}(11)$ & $119.78(16)$ \\
$\mathrm{C}(9)-\mathrm{C}(10)$ & $1.536(3)$ & $\mathrm{C}(3)-\mathrm{C}(2)-\mathrm{C}(1)$ & $130.00(16)$ \\
$\mathrm{C}(10)-\mathrm{C}(11)$ & $1.404(2)$ & $\mathrm{C}(11)-\mathrm{C}(2)-\mathrm{C}(1)$ & $110.21(15)$ \\
$\mathrm{C}(11)-\mathrm{C}(12)$ & $1.387(2)$ & $\mathrm{C}(2)-\mathrm{C}(3)-\mathrm{C}(4)$ & $121.09(16)$ \\
$\mathrm{C}(12)-\mathrm{C}(13)$ & $1.471(2)$ & $\mathrm{C}(3)-\mathrm{C}(4)-\mathrm{C}(9)$ & $119.25(15)$ \\
& $1.387(2)$ & &
\end{tabular}




\begin{tabular}{|c|c|c|c|}
\hline$C(3)-C(4)-C(5)$ & $118.70(15)$ & $C(12)-C(13)-C(14)$ & $120.82(16)$ \\
\hline$C(9)-C(4)-C(5)$ & $121.97(15)$ & $C(13)-C(14)-C(19)$ & $119.00(16)$ \\
\hline$C(4)-C(5)-C(23)$ & $108.19(15)$ & $\mathrm{C}(13)-\mathrm{C}(14)-\mathrm{C}(15)$ & $117.08(15)$ \\
\hline $\mathrm{C}(4)-\mathrm{C}(5)-\mathrm{C}(22)$ & $111.92(14)$ & $\mathrm{C}(19)-\mathrm{C}(14)-\mathrm{C}(15)$ & $123.91(15)$ \\
\hline $\mathrm{C}(23)-\mathrm{C}(5)-\mathrm{C}(22)$ & $108.70(15)$ & $\mathrm{C}(27)-\mathrm{C}(15)-\mathrm{C}(14)$ & $110.94(15)$ \\
\hline$C(4)-C(5)-C(6)$ & $109.78(15)$ & $\mathrm{C}(27)-\mathrm{C}(15)-\mathrm{C}(16)$ & $107.43(15)$ \\
\hline$C(23)-C(5)-C(6)$ & $111.05(16)$ & $\mathrm{C}(14)-\mathrm{C}(15)-\mathrm{C}(16)$ & $111.39(14)$ \\
\hline $\mathrm{C}(22)-\mathrm{C}(5)-\mathrm{C}(6)$ & $107.22(15)$ & $\mathrm{C}(27)-\mathrm{C}(15)-\mathrm{C}(26)$ & $109.53(15)$ \\
\hline$C(7)-C(6)-C(5)$ & $111.71(16)$ & $\mathrm{C}(14)-\mathrm{C}(15)-\mathrm{C}(26)$ & $108.46(14)$ \\
\hline $\mathrm{C}(6)-\mathrm{C}(7)-\mathrm{C}(8)$ & $112.50(15)$ & $C(16)-C(15)-C(26)$ & $109.06(15)$ \\
\hline$C(9)-C(8)-C(24)$ & $111.47(15)$ & $\mathrm{C}(17)-\mathrm{C}(16)-\mathrm{C}(15)$ & $113.44(15)$ \\
\hline$C(9)-C(8)-C(7)$ & $111.36(15)$ & $\mathrm{C}(16)-\mathrm{C}(17)-\mathrm{C}(18)$ & $111.82(15)$ \\
\hline $\mathrm{C}(24)-\mathrm{C}(8)-\mathrm{C}(7)$ & $107.21(15)$ & $\mathrm{C}(28)-\mathrm{C}(18)-\mathrm{C}(19)$ & $112.26(15)$ \\
\hline $\mathrm{C}(9)-\mathrm{C}(8)-\mathrm{C}(25)$ & $108.29(15)$ & $\mathrm{C}(28)-\mathrm{C}(18)-\mathrm{C}(17)$ & $107.76(15)$ \\
\hline $\mathrm{C}(24)-\mathrm{C}(8)-\mathrm{C}(25)$ & 108.61(16) & $\mathrm{C}(19)-\mathrm{C}(18)-\mathrm{C}(17)$ & $109.11(14)$ \\
\hline $\mathrm{C}(7)-\mathrm{C}(8)-\mathrm{C}(25)$ & $109.86(16)$ & $\mathrm{C}(28)-\mathrm{C}(18)-\mathrm{C}(29)$ & $108.11(15)$ \\
\hline$C(10)-C(9)-C(4)$ & $118.96(16)$ & C(19)-C(18)-C(29) & $108.60(15)$ \\
\hline $\mathrm{C}(10)-\mathrm{C}(9)-\mathrm{C}(8)$ & $117.52(15)$ & $\mathrm{C}(17)-\mathrm{C}(18)-\mathrm{C}(29)$ & $111.02(15)$ \\
\hline $\mathrm{C}(4)-\mathrm{C}(9)-\mathrm{C}(8)$ & $123.50(15)$ & $\mathrm{C}(20)-\mathrm{C}(19)-\mathrm{C}(14)$ & 119.31(16) \\
\hline $\mathrm{C}(11)-\mathrm{C}(10)-\mathrm{C}(9)$ & $120.93(16)$ & $\mathrm{C}(20)-\mathrm{C}(19)-\mathrm{C}(18)$ & $119.03(15)$ \\
\hline $\mathrm{C}(10)-\mathrm{C}(11)-\mathrm{C}(2)$ & $119.95(16)$ & $\mathrm{C}(14)-\mathrm{C}(19)-\mathrm{C}(18)$ & $121.61(15)$ \\
\hline$C(10)-C(11)-C(12)$ & $131.64(16)$ & $\mathrm{C}(21)-\mathrm{C}(20)-\mathrm{C}(19)$ & $121.02(16)$ \\
\hline $\mathrm{C}(2)-\mathrm{C}(11)-\mathrm{C}(12)$ & $108.40(15)$ & $\mathrm{C}(20)-\mathrm{C}(21)-\mathrm{C}(12)$ & $119.86(16)$ \\
\hline$C(13)-C(12)-C(21)$ & $119.97(16)$ & $\mathrm{C}(20)-\mathrm{C}(21)-\mathrm{C}(1)$ & $130.23(16)$ \\
\hline $\mathrm{C}(13)-\mathrm{C}(12)-\mathrm{C}(11)$ & $131.18(16)$ & $\mathrm{C}(12)-\mathrm{C}(21)-\mathrm{C}(1)$ & $109.91(15)$ \\
\hline $\mathrm{C}(21)-\mathrm{C}(12)-\mathrm{C}(11)$ & $108.85(15)$ & & \\
\hline
\end{tabular}

Table 8. Anisotropic displacement parameters $\left(\AA^{2} \times 10^{3}\right)$ for sm34. The anisotropic displacement factor exponent takes the form: $-2 \mathrm{p}^{2}\left[\mathrm{~h}^{2} \mathrm{a}^{* 2} \mathrm{U}^{11}+\ldots+2 \mathrm{~h} \mathrm{k} \mathrm{a}^{*} \mathrm{~b}^{*} \mathrm{U}^{12}\right]$

\begin{tabular}{|c|c|c|c|c|c|c|}
\hline & $\mathrm{U}^{11}$ & $\mathrm{U}^{22}$ & $\mathrm{U}^{33}$ & $\mathrm{U}^{23}$ & $\mathrm{U}^{13}$ & $\mathrm{U}^{12}$ \\
\hline $\mathrm{C}(1)$ & $26(1)$ & $12(1)$ & $15(1)$ & $-2(1)$ & $7(1)$ & $-5(1)$ \\
\hline $\mathrm{C}(3)$ & $17(1)$ & $15(1)$ & $10(1)$ & $-1(1)$ & $4(1)$ & $0(1)$ \\
\hline $\mathrm{C}(4)$ & $11(1)$ & $13(1)$ & $11(1)$ & $2(1)$ & $2(1)$ & 1(1) \\
\hline $\mathrm{C}(5)$ & $16(1)$ & $14(1)$ & 11(1) & $1(1)$ & $5(1)$ & $-1(1)$ \\
\hline $\mathrm{C}(8)$ & $18(1)$ & $11(1)$ & $14(1)$ & $-1(1)$ & $5(1)$ & $-2(1)$ \\
\hline $\mathrm{C}(9)$ & $10(1)$ & $11(1)$ & $12(1)$ & $0(1)$ & $0(1)$ & $1(1)$ \\
\hline$C(10)$ & $14(1)$ & $13(1)$ & $9(1)$ & $-1(1)$ & 1(1) & 1(1) \\
\hline $\mathrm{C}(11)$ & $13(1)$ & $13(1)$ & $11(1)$ & $2(1)$ & $2(1)$ & $0(1)$ \\
\hline $\mathrm{C}(12)$ & $13(1)$ & $12(1)$ & $12(1)$ & 1(1) & $2(1)$ & $-2(1)$ \\
\hline$C(13)$ & $14(1)$ & $12(1)$ & $13(1)$ & $-2(1)$ & $2(1)$ & $-1(1)$ \\
\hline$C(17)$ & $16(1)$ & $16(1)$ & $19(1)$ & $3(1)$ & $7(1)$ & $-2(1)$ \\
\hline$C(18)$ & $15(1)$ & $12(1)$ & $13(1)$ & 1(1) & $3(1)$ & $-2(1)$ \\
\hline$C(19)$ & $12(1)$ & $12(1)$ & $12(1)$ & $3(1)$ & $1(1)$ & $0(1)$ \\
\hline $\mathrm{C}(20)$ & $19(1)$ & $12(1)$ & $13(1)$ & $-2(1)$ & $2(1)$ & $-1(1)$ \\
\hline $\mathrm{C}(21)$ & $17(1)$ & $13(1)$ & $11(1)$ & $0(1)$ & $3(1)$ & $0(1)$ \\
\hline $\mathrm{C}(22)$ & $18(1)$ & $19(1)$ & $20(1)$ & $0(1)$ & $9(1)$ & $0(1)$ \\
\hline $\mathrm{C}(23)$ & $23(1)$ & $23(1)$ & $17(1)$ & $6(1)$ & $7(1)$ & $2(1)$ \\
\hline$C(24)$ & $23(1)$ & $13(1)$ & $26(1)$ & $-3(1)$ & 11(1) & $-1(1)$ \\
\hline$C(25)$ & 21(1) & $22(1)$ & $23(1)$ & $-4(1)$ & $-2(1)$ & $-7(1)$ \\
\hline$C(26)$ & $19(1)$ & $17(1)$ & $19(1)$ & 1(1) & $8(1)$ & $2(1)$ \\
\hline$C(27)$ & $18(1)$ & $25(1)$ & $13(1)$ & $-2(1)$ & $4(1)$ & $-2(1)$ \\
\hline $\mathrm{C}(28)$ & $20(1)$ & $23(1)$ & 21(1) & $-2(1)$ & $3(1)$ & $-9(1)$ \\
\hline
\end{tabular}


Table 9. Hydrogen coordinates ( x 104) and isotropic displacement parameters $\left(\AA^{2} \times 10^{3}\right)$ for sm34.

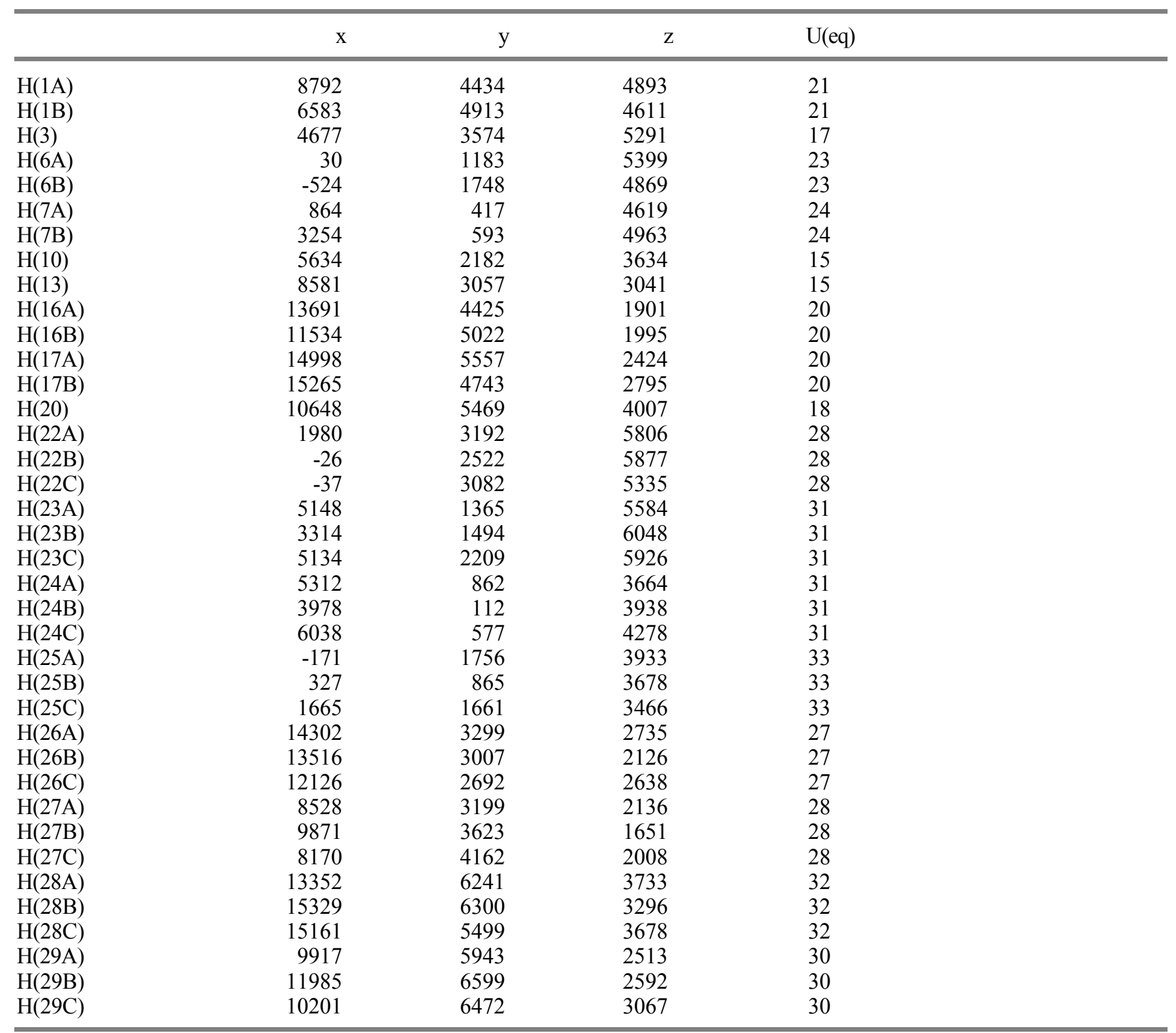


X-Ray Structure Data for $\mathrm{Me}_{2} \mathrm{Si}\left(\eta^{1}-\mathrm{C}_{29} \mathrm{H}_{36}\right)\left(\eta^{1}-\mathrm{N}-{ }^{t} \mathrm{Bu}\right) \mathrm{ZrCl}_{2} \cdot \mathrm{OEt}_{2}$ (1) (sm68)

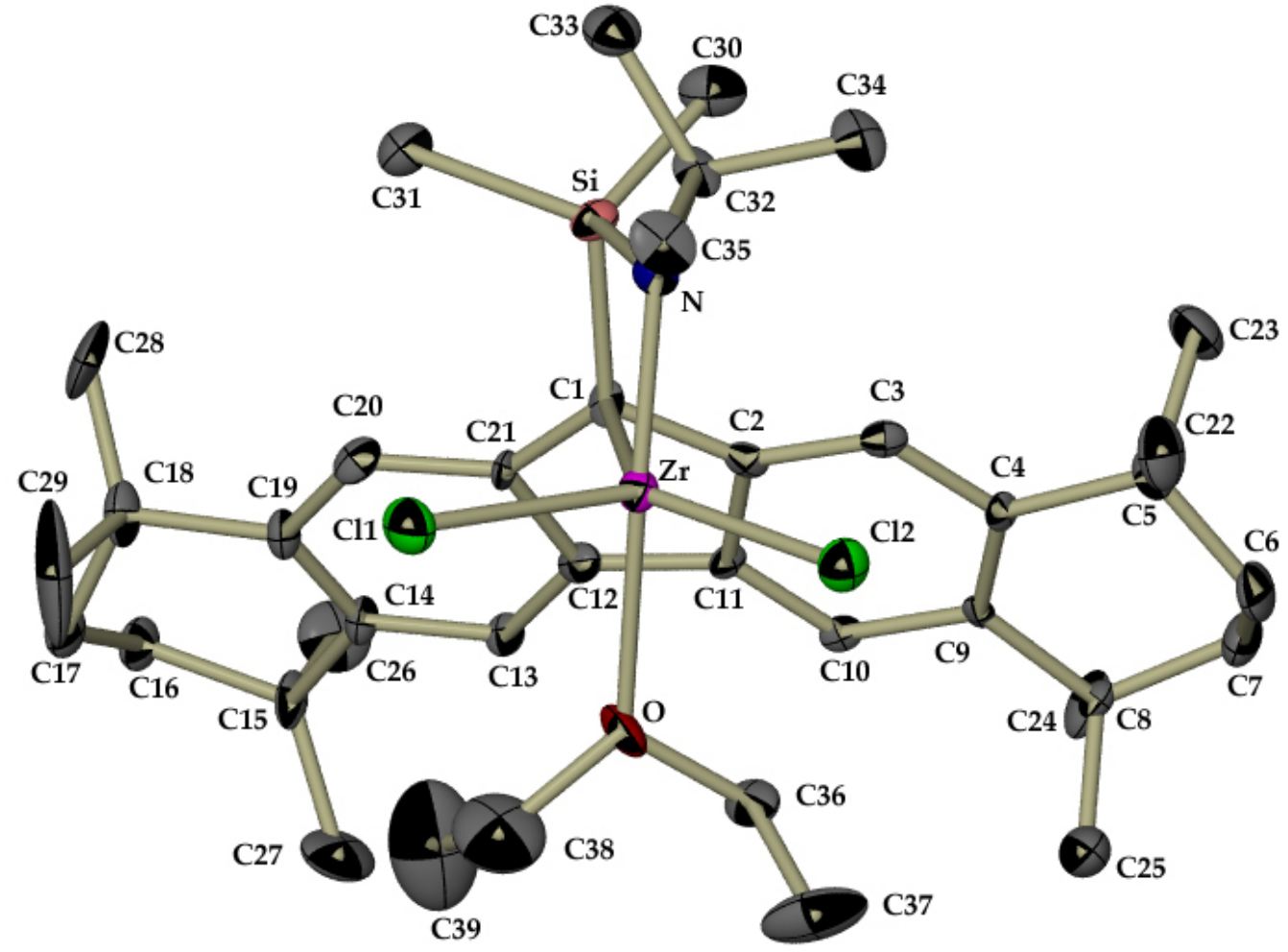

Labeled view of 1 with $50 \%$ probability ellipsoids
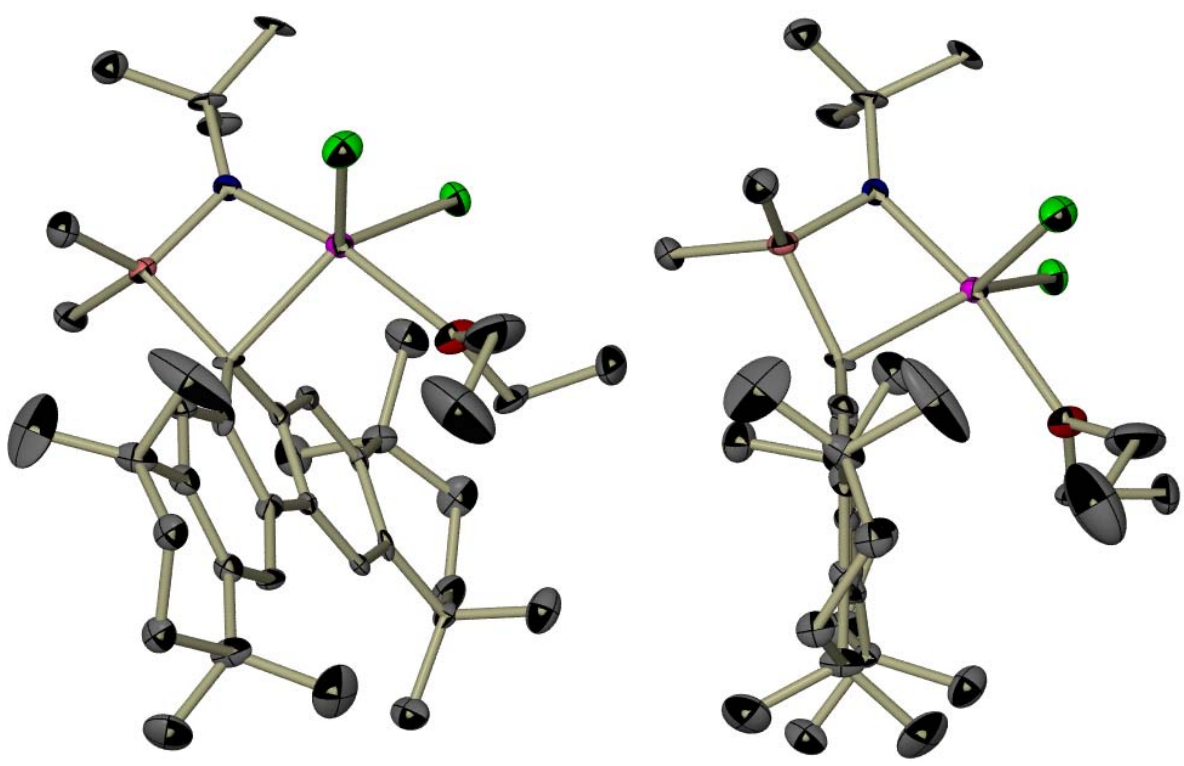

Side-on views of $\mathbf{1}$ with $50 \%$ probability ellipsoids 
Table 10. Crystal data and structure refinement for 1 (sm68).

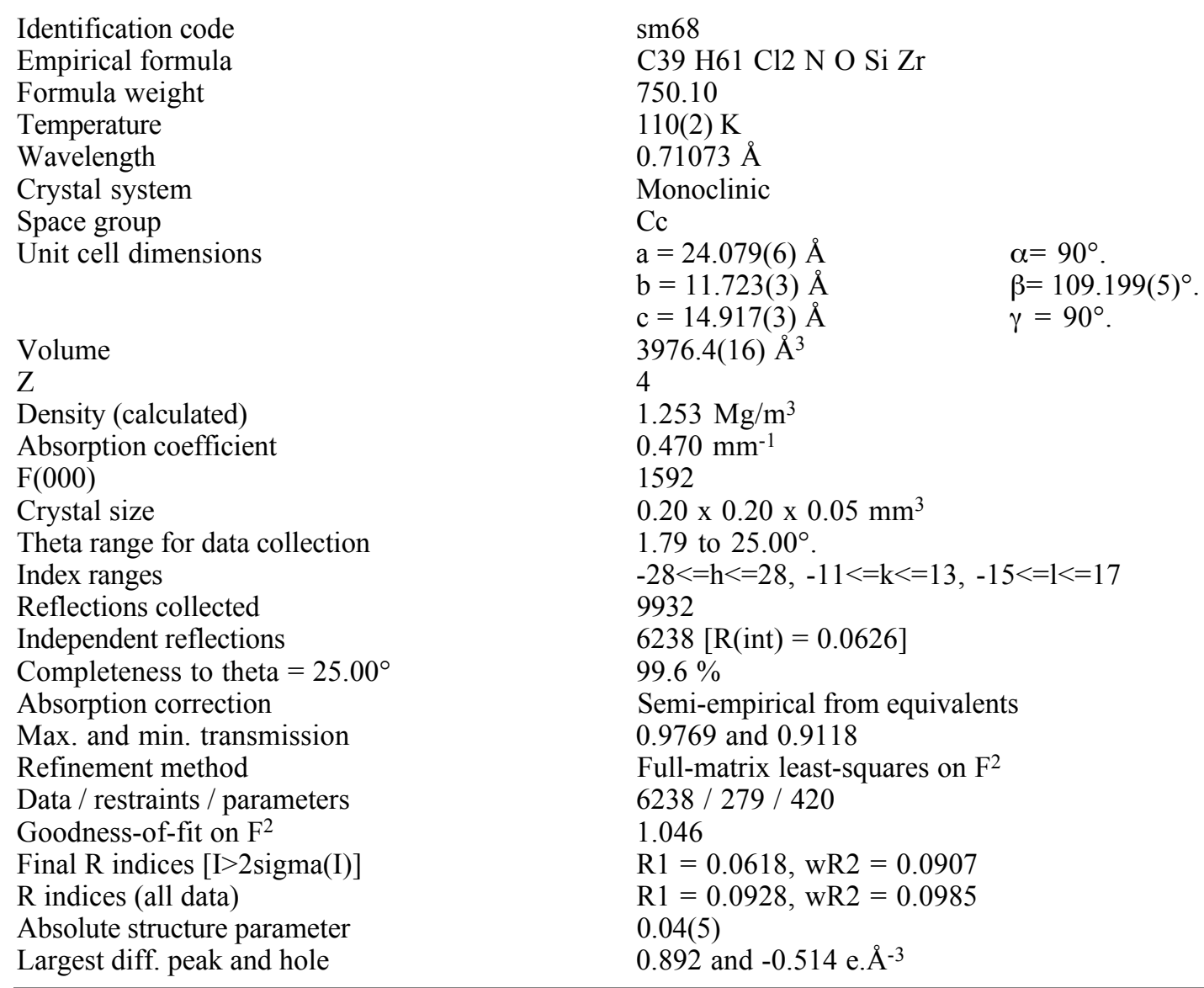

Table 11. Atomic coordinates ( $\mathrm{x} 10^{4}$ ) and equivalent isotropic displacement parameters $\left(\AA^{2} \times 10^{3}\right)$ for sm68. $\mathrm{U}(\mathrm{eq})$ is defined as one third of the trace of the orthogonalized $\mathrm{U}^{\mathrm{ij}}$ tensor.

\begin{tabular}{|c|c|c|c|c|}
\hline & $\mathrm{x}$ & $\mathrm{y}$ & $\mathrm{Z}$ & $\mathrm{U}(\mathrm{eq})$ \\
\hline $\operatorname{Zr}(1)$ & $152(1)$ & $-2997(1)$ & $1802(1)$ & $18(1)$ \\
\hline $\operatorname{Si}(1)$ & $-101(1)$ & $-2882(2)$ & $3554(1)$ & 21(1) \\
\hline $\mathrm{O}(1)$ & $85(2)$ & $-1685(4)$ & 597(3) & $31(1)$ \\
\hline $\mathrm{N}(1)$ & $215(2)$ & $-3885(4)$ & $2986(3)$ & $19(1)$ \\
\hline $\mathrm{Cl}(1)$ & $-723(1)$ & $-3859(2)$ & $760(1)$ & $35(1)$ \\
\hline $\mathrm{C}(2)$ & 493(3) & $-1087(6)$ & 2894(4) & $17(2)$ \\
\hline $\mathrm{C}(3)$ & $1073(3)$ & $-1449(6)$ & $3234(4)$ & $18(2)$ \\
\hline$C(4)$ & $1544(3)$ & $-743(5)$ & $3254(4)$ & $14(2)$ \\
\hline$C(5)$ & $2175(3)$ & $-1166(6)$ & $3715(4)$ & $23(2)$ \\
\hline$C(6)$ & $2598(3)$ & $-454(7)$ & $3400(5)$ & $35(2)$ \\
\hline$C(7)$ & $2510(3)$ & $790(6)$ & $3464(5)$ & $33(2)$ \\
\hline$C(11)$ & $386(3)$ & $56(5)$ & $2543(4)$ & $14(2)$ \\
\hline$C(12)$ & $-238(3)$ & $231(5)$ & $2242(4)$ & $20(2)$ \\
\hline $\mathrm{C}(13)$ & $-584(3)$ & $1200(5)$ & 1891(4) & $18(2)$ \\
\hline$C(14)$ & $-1187(3)$ & $1179(5)$ & $1638(4)$ & $20(2)$ \\
\hline$C(15)$ & $-1527(3)$ & $2285(5)$ & $1283(5)$ & $26(2)$ \\
\hline C(16) & $-2181(4)$ & $2025(10)$ & $718(8)$ & $27(2)$ \\
\hline$C(17)$ & $-2431(5)$ & $1223(9)$ & $1282(9)$ & $27(2)$ \\
\hline
\end{tabular}




\begin{tabular}{lrrrr}
$C\left(16^{\prime}\right)$ & $-2180(9)$ & $2155(17)$ & $1260(18)$ & $27(2)$ \\
$C\left(17^{\prime}\right)$ & $-2420(10)$ & $1025(16)$ & $819(18)$ & $27(2)$ \\
$C(18)$ & $-2148(3)$ & $17(6)$ & $1428(5)$ & $28(2)$ \\
$C(19)$ & $-1477(3)$ & $147(6)$ & $1710(4)$ & $20(2)$ \\
$C(20)$ & $-1133(3)$ & $-817(5)$ & $2069(4)$ & $21(2)$ \\
$C(21)$ & $-518(3)$ & $-819(6)$ & $2350(4)$ & $17(2)$ \\
$C(22)$ & $2250(3)$ & $-2415(6)$ & $3450(5)$ & $38(2)$ \\
$C(23)$ & $2324(3)$ & $-1122(6)$ & $4794(4)$ & $36(2)$ \\
$C(24)$ & $1820(3)$ & $2395(6)$ & $3182(6)$ & $35(2)$ \\
$C(25)$ & $1882(3)$ & $1277(7)$ & $1810(4)$ & $39(2)$ \\
$C(26)$ & $-1414(4)$ & $3128(7)$ & $2089(5)$ & $48(2)$ \\
$C(27)$ & $-1320(4)$ & $2825(7)$ & $498(5)$ & $53(3)$ \\
$C(28)$ & $-2315(4)$ & $-392(9)$ & $2257(6)$ & $77(3)$ \\
$C(29)$ & $-2361(4)$ & $-778(9)$ & $612(7)$ & $96(5)$ \\
$C(30)$ & $335(3)$ & $-2555(6)$ & $4817(4)$ & $33(2)$ \\
$C(31)$ & $-878(3)$ & $-3238(6)$ & $3471(5)$ & $32(2)$ \\
$C(32)$ & $414(3)$ & $-5048(5)$ & $3364(5)$ & $22(2)$ \\
$C(33)$ & $59(3)$ & $-5496(6)$ & $3954(5)$ & $31(2)$ \\
$C(34)$ & $335(3)$ & $-5852(5)$ & $2537(5)$ & $31(2)$ \\
$C(35)$ & $1057(3)$ & $-4975(6)$ & $3966(5)$ & $32(2)$ \\
$C(36)$ & $596(3)$ & $-1006(6)$ & $631(5)$ & $29(2)$ \\
$C(37)$ & $893(4)$ & $-1374(7)$ & $-86(5)$ & $64(3)$ \\
$C(38)$ & $-409(5)$ & $-1512(8)$ & $-318(7)$ & $71(3)$ \\
$C(39)$ & $-726(5)$ & $-552(10)$ & $-250(8)$ & $111(5)$ \\
\hline
\end{tabular}

Table 12. Bond lengths $[\AA]$ and angles $\left[{ }^{\circ}\right]$ for sm68.

\begin{tabular}{|c|c|c|c|}
\hline $\mathrm{Zr}(1)-\mathrm{N}(1)$ & $2.013(5)$ & $\mathrm{C}(10)-\mathrm{C}(11)$ & $1.366(8)$ \\
\hline $\operatorname{Zr}(1)-C(1)$ & $2.299(7)$ & $\mathrm{C}(10)-\mathrm{H}(10)$ & 0.9500 \\
\hline $\mathrm{Zr}(1)-\mathrm{O}(1)$ & $2.330(5)$ & C(11)-C(12) & $1.434(8)$ \\
\hline $\operatorname{Zr}(1)-\mathrm{Cl}(1)$ & $2.3918(18)$ & $\mathrm{C}(12)-\mathrm{C}(13)$ & $1.404(8)$ \\
\hline $\operatorname{Zr}(1)-\mathrm{Cl}(2)$ & $2.4155(19)$ & $C(12)-C(21)$ & $1.437(9)$ \\
\hline $\operatorname{Zr}(1)-C(2)$ & $2.731(6)$ & C(13)-C(14) & $1.375(8)$ \\
\hline $\mathrm{Zr}(1)-\operatorname{Si}(1)$ & $2.8759(18)$ & C(13)-H(13) & 0.9500 \\
\hline $\mathrm{Si}(1)-\mathrm{N}(1)$ & $1.761(5)$ & C(14)-C(19) & $1.418(9)$ \\
\hline $\operatorname{Si}(1)-C(30)$ & $1.870(6)$ & C(14)-C(15) & $1.533(9)$ \\
\hline $\mathrm{Si}(1)-\mathrm{C}(1)$ & $1.875(7)$ & C(15)-C(26) & $1.510(9)$ \\
\hline $\mathrm{Si}(1)-\mathrm{C}(31)$ & $1.881(7)$ & C(15)-C(27) & $1.551(9)$ \\
\hline $\mathrm{O}(1)-\mathrm{C}(36)$ & $1.452(8)$ & C(15)-C(16) & $1.552(11)$ \\
\hline $\mathrm{O}(1)-\mathrm{C}(38)$ & $1.500(10)$ & $\mathrm{C}(15)-\mathrm{C}\left(16^{\prime}\right)$ & $1.57(2)$ \\
\hline $\mathrm{N}(1)-\mathrm{C}(32)$ & $1.493(7)$ & $C(16)-C(17)$ & $1.511(12)$ \\
\hline $\mathrm{C}(1)-\mathrm{C}(21)$ & $1.441(9)$ & C(16)-H(16A) & 0.9900 \\
\hline $\mathrm{C}(1)-\mathrm{C}(2)$ & $1.468(9)$ & $\mathrm{C}(16)-\mathrm{H}(16 \mathrm{~B})$ & 0.9900 \\
\hline $\mathrm{C}(2)-\mathrm{C}(3)$ & $1.386(9)$ & $\mathrm{C}(17)-\mathrm{C}(18)$ & $1.553(12)$ \\
\hline $\mathrm{C}(2)-\mathrm{C}(11)$ & $1.431(8)$ & C(17)-H(17A) & 0.9900 \\
\hline$C(3)-C(4)$ & $1.396(8)$ & C(17)-H(17B) & 0.9900 \\
\hline $\mathrm{C}(3)-\mathrm{H}(3)$ & 0.9500 & $C\left(16^{\prime}\right)-C\left(17^{\prime}\right)$ & $1.507(17)$ \\
\hline $\mathrm{C}(4)-\mathrm{C}(9)$ & $1.418(8)$ & $\mathrm{C}\left(16^{\prime}\right)-\mathrm{H}(16 \mathrm{C})$ & 0.9900 \\
\hline$C(4)-C(5)$ & $1.530(8)$ & $\mathrm{C}\left(16^{\prime}\right)-\mathrm{H}(16 \mathrm{D})$ & 0.9900 \\
\hline$C(5)-C(6)$ & $1.507(9)$ & $C\left(17^{\prime}\right)-C(18)$ & $1.50(2)$ \\
\hline$C(5)-C(23)$ & $1.529(8)$ & $\mathrm{C}\left(17^{\prime}\right)-\mathrm{H}(17 \mathrm{C})$ & 0.9900 \\
\hline $\mathrm{C}(5)-\mathrm{C}(22)$ & $1.542(9)$ & $\mathrm{C}\left(17^{\prime}\right)-\mathrm{H}(17 \mathrm{D})$ & 0.9900 \\
\hline$C(6)-C(7)$ & $1.481(10)$ & $\mathrm{C}(18)-\mathrm{C}(29)$ & $1.484(10)$ \\
\hline $\mathrm{C}(6)-\mathrm{H}(6 \mathrm{~A})$ & 0.9900 & $C(18)-C(28)$ & $1.499(10)$ \\
\hline $\mathrm{C}(6)-\mathrm{H}(6 \mathrm{~B})$ & 0.9900 & C(18)-C(19) & $1.538(9)$ \\
\hline $\mathrm{C}(7)-\mathrm{C}(8)$ & $1.522(8)$ & $\mathrm{C}(19)-\mathrm{C}(20)$ & $1.399(8)$ \\
\hline $\mathrm{C}(7)-\mathrm{H}(7 \mathrm{~A})$ & 0.9900 & $C(20)-C(21)$ & $1.401(9)$ \\
\hline $\mathrm{C}(7)-\mathrm{H}(7 \mathrm{~B})$ & 0.9900 & $\mathrm{C}(20)-\mathrm{H}(20)$ & 0.9500 \\
\hline$C(8)-C(9)$ & $1.517(8)$ & $\mathrm{C}(22)-\mathrm{H}(22 \mathrm{~A})$ & 0.9800 \\
\hline $\mathrm{C}(8)-\mathrm{C}(24)$ & $1.524(9)$ & $\mathrm{C}(22)-\mathrm{H}(22 \mathrm{~B})$ & 0.9800 \\
\hline$C(8)-C(25)$ & $1.533(8)$ & $\mathrm{C}(22)-\mathrm{H}(22 \mathrm{C})$ & 0.9800 \\
\hline$C(9)-C(10)$ & $1.394(8)$ & $\mathrm{C}(23)-\mathrm{H}(23 \mathrm{~A})$ & 0.9800 \\
\hline
\end{tabular}




\begin{tabular}{|c|c|}
\hline $\mathrm{C}(23)-\mathrm{H}(23 \mathrm{~B})$ & 0.9800 \\
\hline $\mathrm{C}(23)-\mathrm{H}(23 \mathrm{C})$ & 0.9800 \\
\hline $\mathrm{C}(24)-\mathrm{H}(24 \mathrm{~A})$ & 0.9800 \\
\hline $\mathrm{C}(24)-\mathrm{H}(24 \mathrm{~B})$ & 0.9800 \\
\hline $\mathrm{C}(24)-\mathrm{H}(24 \mathrm{C})$ & 0.9800 \\
\hline $\mathrm{C}(25)-\mathrm{H}(25 \mathrm{~A})$ & 0.9800 \\
\hline $\mathrm{C}(25)-\mathrm{H}(25 \mathrm{~B})$ & 0.9800 \\
\hline $\mathrm{C}(25)-\mathrm{H}(25 \mathrm{C})$ & 0.9800 \\
\hline $\mathrm{C}(26)-\mathrm{H}(26 \mathrm{~A})$ & 0.9599 \\
\hline $\mathrm{C}(26)-\mathrm{H}(26 \mathrm{~B})$ & 0.9601 \\
\hline $\mathrm{C}(26)-\mathrm{H}(26 \mathrm{C})$ & 0.9601 \\
\hline $\mathrm{C}(27)-\mathrm{H}(27 \mathrm{~A})$ & 0.9599 \\
\hline $\mathrm{C}(27)-\mathrm{H}(27 \mathrm{~B})$ & 0.9601 \\
\hline $\mathrm{C}(27)-\mathrm{H}(27 \mathrm{C})$ & 0.9600 \\
\hline $\mathrm{C}(28)-\mathrm{H}(28 \mathrm{~A})$ & 0.9800 \\
\hline $\mathrm{C}(28)-\mathrm{H}(28 \mathrm{~B})$ & 0.9800 \\
\hline $\mathrm{C}(28)-\mathrm{H}(28 \mathrm{C})$ & 0.9800 \\
\hline C(29)-H(29A) & 0.9800 \\
\hline $\mathrm{C}(29)-\mathrm{H}(29 \mathrm{~B})$ & 0.9800 \\
\hline $\mathrm{C}(29)-\mathrm{H}(29 \mathrm{C})$ & 0.9800 \\
\hline $\mathrm{C}(30)-\mathrm{H}(30 \mathrm{~A})$ & 0.9800 \\
\hline $\mathrm{C}(30)-\mathrm{H}(30 \mathrm{~B})$ & 0.9800 \\
\hline $\mathrm{C}(30)-\mathrm{H}(30 \mathrm{C})$ & 0.9800 \\
\hline $\mathrm{C}(31)-\mathrm{H}(31 \mathrm{~A})$ & 0.9800 \\
\hline $\mathrm{C}(31)-\mathrm{H}(31 \mathrm{~B})$ & 0.9800 \\
\hline $\mathrm{C}(31)-\mathrm{H}(31 \mathrm{C})$ & 0.9800 \\
\hline $\mathrm{C}(32)-\mathrm{C}(33)$ & $1.509(9)$ \\
\hline$C(32)-C(34)$ & $1.514(8)$ \\
\hline C(32)-C(35) & $1.515(9)$ \\
\hline $\mathrm{C}(33)-\mathrm{H}(33 \mathrm{~A})$ & 0.9800 \\
\hline $\mathrm{C}(33)-\mathrm{H}(33 \mathrm{~B})$ & 0.9800 \\
\hline $\mathrm{C}(33)-\mathrm{H}(33 \mathrm{C})$ & 0.9800 \\
\hline $\mathrm{C}(34)-\mathrm{H}(34 \mathrm{~A})$ & 0.9800 \\
\hline $\mathrm{C}(34)-\mathrm{H}(34 \mathrm{~B})$ & 0.9800 \\
\hline $\mathrm{C}(34)-\mathrm{H}(34 \mathrm{C})$ & 0.9800 \\
\hline $\mathrm{C}(35)-\mathrm{H}(35 \mathrm{~A})$ & 0.9800 \\
\hline $\mathrm{C}(35)-\mathrm{H}(35 \mathrm{~B})$ & 0.9800 \\
\hline $\mathrm{C}(35)-\mathrm{H}(35 \mathrm{C})$ & 0.9800 \\
\hline $\mathrm{C}(36)-\mathrm{C}(37)$ & $1.531(10)$ \\
\hline $\mathrm{C}(36)-\mathrm{H}(36 \mathrm{~A})$ & 0.9900 \\
\hline $\mathrm{C}(36)-\mathrm{H}(36 \mathrm{~B})$ & 0.9900 \\
\hline $\mathrm{C}(37)-\mathrm{H}(37 \mathrm{~A})$ & 0.9800 \\
\hline $\mathrm{C}(37)-\mathrm{H}(37 \mathrm{~B})$ & 0.9800 \\
\hline $\mathrm{C}(37)-\mathrm{H}(37 \mathrm{C})$ & 0.9800 \\
\hline $\mathrm{C}(38)-\mathrm{C}(39)$ & $1.383(12)$ \\
\hline $\mathrm{C}(38)-\mathrm{H}(38 \mathrm{~A})$ & 0.9900 \\
\hline $\mathrm{C}(38)-\mathrm{H}(38 \mathrm{~B})$ & 0.9900 \\
\hline $\mathrm{C}(39)-\mathrm{H}(39 \mathrm{~A})$ & 0.9800 \\
\hline C(39)-H(39B) & 0.9800 \\
\hline C(39)-H(39C) & 0.9800 \\
\hline $\mathrm{N}(1)-\mathrm{Zr}(1)-\mathrm{C}(1)$ & $76.8(2)$ \\
\hline $\mathrm{N}(1)-\mathrm{Zr}(1)-\mathrm{O}(1)$ & 169.87(19) \\
\hline $\mathrm{C}(1)-\mathrm{Zr}(1)-\mathrm{O}(1)$ & 93.40(18) \\
\hline $\mathrm{N}(1)-\mathrm{Zr}(1)-\mathrm{Cl}(1)$ & $98.47(15)$ \\
\hline $\mathrm{C}(1)-\mathrm{Zr}(1)-\mathrm{Cl}(1)$ & $110.87(18)$ \\
\hline $\mathrm{O}(1)-\mathrm{Zr}(1)-\mathrm{Cl}(1)$ & 87.40(12) \\
\hline $\mathrm{N}(1)-\mathrm{Zr}(1)-\mathrm{Cl}(2)$ & $98.48(15)$ \\
\hline $\mathrm{C}(1)-\mathrm{Zr}(1)-\mathrm{Cl}(2)$ & $136.16(18)$ \\
\hline $\mathrm{O}(1)-\mathrm{Zr}(1)-\mathrm{Cl}(2)$ & 86.70(14) \\
\hline $\mathrm{Cl}(1)-\mathrm{Zr}(1)-\mathrm{Cl}(2)$ & $112.92(7)$ \\
\hline $\mathrm{N}(1)-\operatorname{Zr}(1)-\mathrm{C}(2)$ & 88.83(19) \\
\hline $\mathrm{C}(1)-\mathrm{Zr}(1)-\mathrm{C}(2)$ & $32.5(2)$ \\
\hline $\mathrm{O}(1)-\mathrm{Zr}(1)-\mathrm{C}(2)$ & 81.39(18) \\
\hline $\mathrm{Cl}(1)-\mathrm{Zr}(1)-\mathrm{C}(2)$ & $139.60(15)$ \\
\hline
\end{tabular}

\begin{tabular}{|c|c|}
\hline $\mathrm{Cl}(2)-\mathrm{Zr}(1)-\mathrm{C}(2)$ & $105.06(15)$ \\
\hline $\mathrm{N}(1)-\operatorname{Zr}(1)-\mathrm{Si}(1)$ & $37.21(15)$ \\
\hline $\mathrm{C}(1)-\mathrm{Zr}(1)-\mathrm{Si}(1)$ & $40.60(17)$ \\
\hline $\mathrm{O}(1)-\operatorname{Zr}(1)-\operatorname{Si}(1)$ & $133.54(14)$ \\
\hline $\mathrm{Cl}(1)-\mathrm{Zr}(1)-\mathrm{Si}(1)$ & $101.86(6)$ \\
\hline $\mathrm{Cl}(2)-\mathrm{Zr}(1)-\mathrm{Si}(1)$ & $128.11(6)$ \\
\hline $\mathrm{C}(2)-\mathrm{Zr}(1)-\mathrm{Si}(1)$ & $62.08(14)$ \\
\hline $\mathrm{N}(1)-\mathrm{Si}(1)-\mathrm{C}(30)$ & $115.6(3)$ \\
\hline $\mathrm{N}(1)-\mathrm{Si}(1)-\mathrm{C}(1)$ & $95.3(3)$ \\
\hline $\mathrm{C}(30)-\mathrm{Si}(1)-\mathrm{C}(1)$ & $110.7(3)$ \\
\hline $\mathrm{N}(1)-\mathrm{Si}(1)-\mathrm{C}(31)$ & $113.5(3)$ \\
\hline $\mathrm{C}(30)-\mathrm{Si}(1)-\mathrm{C}(31)$ & $109.2(3)$ \\
\hline $\mathrm{C}(1)-\mathrm{Si}(1)-\mathrm{C}(31)$ & $111.9(3)$ \\
\hline $\mathrm{N}(1)-\operatorname{Si}(1)-\operatorname{Zr}(1)$ & $43.72(16)$ \\
\hline$C(30)-\operatorname{Si}(1)-\operatorname{Zr}(1)$ & $135.1(2)$ \\
\hline $\mathrm{C}(1)-\mathrm{Si}(1)-\operatorname{Zr}(1)$ & $52.9(2)$ \\
\hline C(31)-Si(1)-Zr(1) & $115.7(2)$ \\
\hline $\mathrm{C}(36)-\mathrm{O}(1)-\mathrm{C}(38)$ & $111.5(6)$ \\
\hline $\mathrm{C}(36)-\mathrm{O}(1)-\mathrm{Zr}(1)$ & $119.5(4)$ \\
\hline $\mathrm{C}(38)-\mathrm{O}(1)-\operatorname{Zr}(1)$ & $128.6(5)$ \\
\hline $\mathrm{C}(32)-\mathrm{N}(1)-\mathrm{Si}(1)$ & $124.3(4)$ \\
\hline $\mathrm{C}(32)-\mathrm{N}(1)-\operatorname{Zr}(1)$ & $136.5(4)$ \\
\hline $\operatorname{Si}(1)-N(1)-\operatorname{Zr}(1)$ & $99.1(2)$ \\
\hline $\mathrm{C}(21)-\mathrm{C}(1)-\mathrm{C}(2)$ & $105.8(5)$ \\
\hline $\mathrm{C}(21)-\mathrm{C}(1)-\mathrm{Si}(1)$ & $127.7(5)$ \\
\hline $\mathrm{C}(2)-\mathrm{C}(1)-\mathrm{Si}(1)$ & $119.4(5)$ \\
\hline $\mathrm{C}(21)-\mathrm{C}(1)-\operatorname{Zr}(1)$ & $119.8(5)$ \\
\hline $\mathrm{C}(2)-\mathrm{C}(1)-\mathrm{Zr}(1)$ & $90.2(4)$ \\
\hline $\mathrm{Si}(1)-\mathrm{C}(1)-\operatorname{Zr}(1)$ & $86.5(2)$ \\
\hline $\mathrm{C}(3)-\mathrm{C}(2)-\mathrm{C}(11)$ & $117.3(6)$ \\
\hline $\mathrm{C}(3)-\mathrm{C}(2)-\mathrm{C}(1)$ & $133.2(6)$ \\
\hline $\mathrm{C}(11)-\mathrm{C}(2)-\mathrm{C}(1)$ & $109.4(6)$ \\
\hline $\mathrm{C}(3)-\mathrm{C}(2)-\mathrm{Zr}(1)$ & $92.1(4)$ \\
\hline $\mathrm{C}(11)-\mathrm{C}(2)-\operatorname{Zr}(1)$ & $124.5(4)$ \\
\hline $\mathrm{C}(1)-\mathrm{C}(2)-\mathrm{Zr}(1)$ & $57.3(3)$ \\
\hline $\mathrm{C}(2)-\mathrm{C}(3)-\mathrm{C}(4)$ & $122.7(6)$ \\
\hline $\mathrm{C}(2)-\mathrm{C}(3)-\mathrm{H}(3)$ & 118.7 \\
\hline $\mathrm{C}(4)-\mathrm{C}(3)-\mathrm{H}(3)$ & 118.7 \\
\hline $\mathrm{C}(3)-\mathrm{C}(4)-\mathrm{C}(9)$ & $119.0(6)$ \\
\hline$C(3)-C(4)-C(5)$ & $119.8(6)$ \\
\hline$C(9)-C(4)-C(5)$ & $121.1(6)$ \\
\hline$C(6)-C(5)-C(23)$ & $111.1(6)$ \\
\hline$C(6)-C(5)-C(4)$ & $110.7(5)$ \\
\hline$C(23)-C(5)-C(4)$ & $108.5(5)$ \\
\hline$C(6)-C(5)-C(22)$ & $107.3(6)$ \\
\hline$C(23)-C(5)-C(22)$ & $107.2(6)$ \\
\hline$C(4)-C(5)-C(22)$ & $112.1(5)$ \\
\hline$C(7)-C(6)-C(5)$ & $113.6(6)$ \\
\hline $\mathrm{C}(7)-\mathrm{C}(6)-\mathrm{H}(6 \mathrm{~A})$ & 108.9 \\
\hline $\mathrm{C}(5)-\mathrm{C}(6)-\mathrm{H}(6 \mathrm{~A})$ & 108.9 \\
\hline $\mathrm{C}(7)-\mathrm{C}(6)-\mathrm{H}(6 \mathrm{~B})$ & 108.9 \\
\hline $\mathrm{C}(5)-\mathrm{C}(6)-\mathrm{H}(6 \mathrm{~B})$ & 108.9 \\
\hline $\mathrm{H}(6 \mathrm{~A})-\mathrm{C}(6)-\mathrm{H}(6 \mathrm{~B})$ & 107.7 \\
\hline$C(6)-C(7)-C(8)$ & $113.6(6)$ \\
\hline $\mathrm{C}(6)-\mathrm{C}(7)-\mathrm{H}(7 \mathrm{~A})$ & 108.9 \\
\hline $\mathrm{C}(8)-\mathrm{C}(7)-\mathrm{H}(7 \mathrm{~A})$ & 108.9 \\
\hline $\mathrm{C}(6)-\mathrm{C}(7)-\mathrm{H}(7 \mathrm{~B})$ & 108.9 \\
\hline $\mathrm{C}(8)-\mathrm{C}(7)-\mathrm{H}(7 \mathrm{~B})$ & 108.9 \\
\hline $\mathrm{H}(7 \mathrm{~A})-\mathrm{C}(7)-\mathrm{H}(7 \mathrm{~B})$ & 107.7 \\
\hline $\mathrm{C}(9)-\mathrm{C}(8)-\mathrm{C}(7)$ & $111.3(5)$ \\
\hline $\mathrm{C}(9)-\mathrm{C}(8)-\mathrm{C}(24)$ & $112.2(5)$ \\
\hline $\mathrm{C}(7)-\mathrm{C}(8)-\mathrm{C}(24)$ & $107.2(5)$ \\
\hline $\mathrm{C}(9)-\mathrm{C}(8)-\mathrm{C}(25)$ & $108.2(5)$ \\
\hline $\mathrm{C}(7)-\mathrm{C}(8)-\mathrm{C}(25)$ & $109.7(5)$ \\
\hline
\end{tabular}




\begin{tabular}{|c|c|}
\hline$C(24)-C(8)-C(25)$ & $108.1(6)$ \\
\hline$C(10)-C(9)-C(4)$ & $118.5(6)$ \\
\hline $\mathrm{C}(10)-\mathrm{C}(9)-\mathrm{C}(8)$ & $118.5(6)$ \\
\hline $\mathrm{C}(4)-\mathrm{C}(9)-\mathrm{C}(8)$ & $123.0(5)$ \\
\hline $\mathrm{C}(11)-\mathrm{C}(10)-\mathrm{C}(9)$ & $122.0(6)$ \\
\hline $\mathrm{C}(11)-\mathrm{C}(10)-\mathrm{H}(10)$ & 119.0 \\
\hline $\mathrm{C}(9)-\mathrm{C}(10)-\mathrm{H}(10)$ & 119.0 \\
\hline $\mathrm{C}(10)-\mathrm{C}(11)-\mathrm{C}(2)$ & $120.5(6)$ \\
\hline $\mathrm{C}(10)-\mathrm{C}(11)-\mathrm{C}(12)$ & $132.6(6)$ \\
\hline $\mathrm{C}(2)-\mathrm{C}(11)-\mathrm{C}(12)$ & $107.0(6)$ \\
\hline$C(13)-C(12)-C(11)$ & $131.6(6)$ \\
\hline$C(13)-C(12)-C(21)$ & $119.5(6)$ \\
\hline$C(11)-C(12)-C(21)$ & $108.9(6)$ \\
\hline $\mathrm{C}(14)-\mathrm{C}(13)-\mathrm{C}(12)$ & $122.0(6)$ \\
\hline $\mathrm{C}(14)-\mathrm{C}(13)-\mathrm{H}(13)$ & 119.0 \\
\hline $\mathrm{C}(12)-\mathrm{C}(13)-\mathrm{H}(13)$ & 119.0 \\
\hline$C(13)-C(14)-C(19)$ & $119.8(6)$ \\
\hline$C(13)-C(14)-C(15)$ & $118.4(6)$ \\
\hline $\mathrm{C}(19)-\mathrm{C}(14)-\mathrm{C}(15)$ & $121.9(6)$ \\
\hline$C(26)-C(15)-C(14)$ & $110.0(6)$ \\
\hline$C(26)-C(15)-C(27)$ & $108.3(6)$ \\
\hline$C(14)-C(15)-C(27)$ & $109.8(6)$ \\
\hline$C(26)-C(15)-C(16)$ & $116.5(7)$ \\
\hline$C(14)-C(15)-C(16)$ & $110.6(6)$ \\
\hline$C(27)-C(15)-C(16)$ & $101.1(6)$ \\
\hline $\mathrm{C}(26)-\mathrm{C}(15)-\mathrm{C}\left(16^{\prime}\right)$ & $90.0(9)$ \\
\hline$C(14)-C(15)-C\left(16^{\prime}\right)$ & $110.1(10)$ \\
\hline $\mathrm{C}(27)-\mathrm{C}(15)-\mathrm{C}\left(16^{\prime}\right)$ & $126.4(11)$ \\
\hline $\mathrm{C}(16)-\mathrm{C}(15)-\mathrm{C}\left(16^{\prime}\right)$ & $30.5(7)$ \\
\hline C(17)-C(16)-C(15) & $109.8(8)$ \\
\hline $\mathrm{C}(17)-\mathrm{C}(16)-\mathrm{H}(16 \mathrm{~A})$ & 109.7 \\
\hline $\mathrm{C}(15)-\mathrm{C}(16)-\mathrm{H}(16 \mathrm{~A})$ & 109.7 \\
\hline $\mathrm{C}(17)-\mathrm{C}(16)-\mathrm{H}(16 \mathrm{~B})$ & 109.7 \\
\hline $\mathrm{C}(15)-\mathrm{C}(16)-\mathrm{H}(16 \mathrm{~B})$ & 109.7 \\
\hline $\mathrm{H}(16 \mathrm{~A})-\mathrm{C}(16)-\mathrm{H}(16 \mathrm{~B})$ & 108.2 \\
\hline$C(16)-C(17)-C(18)$ & $114.1(10)$ \\
\hline $\mathrm{C}(16)-\mathrm{C}(17)-\mathrm{H}(17 \mathrm{~A})$ & 108.7 \\
\hline $\mathrm{C}(18)-\mathrm{C}(17)-\mathrm{H}(17 \mathrm{~A})$ & 108.7 \\
\hline $\mathrm{C}(16)-\mathrm{C}(17)-\mathrm{H}(17 \mathrm{~B})$ & 108.7 \\
\hline $\mathrm{C}(18)-\mathrm{C}(17)-\mathrm{H}(17 \mathrm{~B})$ & 108.7 \\
\hline $\mathrm{H}(17 \mathrm{~A})-\mathrm{C}(17)-\mathrm{H}(17 \mathrm{~B})$ & 107.6 \\
\hline$C\left(17^{\prime}\right)-C\left(16^{\prime}\right)-C(15)$ & $109.4(17)$ \\
\hline $\mathrm{C}\left(17^{\prime}\right)-\mathrm{C}\left(16^{\prime}\right)-\mathrm{H}(16 \mathrm{C})$ & 109.8 \\
\hline $\mathrm{C}(15)-\mathrm{C}\left(16^{\prime}\right)-\mathrm{H}(16 \mathrm{C})$ & 109.8 \\
\hline $\mathrm{C}\left(17^{\prime}\right)-\mathrm{C}\left(16^{\prime}\right)-\mathrm{H}(16 \mathrm{D})$ & 109.8 \\
\hline $\mathrm{C}(15)-\mathrm{C}\left(16^{\prime}\right)-\mathrm{H}(16 \mathrm{D})$ & 109.8 \\
\hline $\mathrm{H}(16 \mathrm{C})-\mathrm{C}\left(16^{\prime}\right)-\mathrm{H}(16 \mathrm{D})$ & 108.2 \\
\hline$C(18)-C\left(17^{\prime}\right)-C\left(16^{\prime}\right)$ & $113.6(19)$ \\
\hline $\mathrm{C}(18)-\mathrm{C}\left(17^{\prime}\right)-\mathrm{H}(17 \mathrm{C})$ & 108.9 \\
\hline $\mathrm{C}\left(16^{\prime}\right)-\mathrm{C}\left(17^{\prime}\right)-\mathrm{H}(17 \mathrm{C})$ & 108.9 \\
\hline $\mathrm{C}(18)-\mathrm{C}\left(17^{\prime}\right)-\mathrm{H}(17 \mathrm{D})$ & 108.9 \\
\hline $\mathrm{C}\left(16^{\prime}\right)-\mathrm{C}\left(17^{\prime}\right)-\mathrm{H}(17 \mathrm{D})$ & 108.9 \\
\hline $\mathrm{H}(17 \mathrm{C})-\mathrm{C}\left(17^{\prime}\right)-\mathrm{H}(17 \mathrm{D})$ & 107.7 \\
\hline$C(29)-C(18)-C(28)$ & $111.2(8)$ \\
\hline $\mathrm{C}(29)-\mathrm{C}(18)-\mathrm{C}\left(17^{\prime}\right)$ & $91.9(10)$ \\
\hline $\mathrm{C}(28)-\mathrm{C}(18)-\mathrm{C}\left(17^{\prime}\right)$ & $124.7(12)$ \\
\hline C(29)-C(18)-C(19) & $109.6(6)$ \\
\hline $\mathrm{C}(28)-\mathrm{C}(18)-\mathrm{C}(19)$ & $110.4(6)$ \\
\hline $\mathrm{C}\left(17^{\prime}\right)-\mathrm{C}(18)-\mathrm{C}(19)$ & $107.3(10)$ \\
\hline $\mathrm{C}(29)-\mathrm{C}(18)-\mathrm{C}(17)$ & $116.0(8)$ \\
\hline $\mathrm{C}(28)-\mathrm{C}(18)-\mathrm{C}(17)$ & $100.5(7)$ \\
\hline $\mathrm{C}\left(17^{\prime}\right)-\mathrm{C}(18)-\mathrm{C}(17)$ & $27.9(8)$ \\
\hline $\mathrm{C}(19)-\mathrm{C}(18)-\mathrm{C}(17)$ & $108.7(6)$ \\
\hline $\mathrm{C}(20)-\mathrm{C}(19)-\mathrm{C}(14)$ & $118.3(6)$ \\
\hline
\end{tabular}

\begin{tabular}{|c|c|}
\hline$C(20)-C(19)-C(18)$ & $117.4(6)$ \\
\hline $\mathrm{C}(14)-\mathrm{C}(19)-\mathrm{C}(18)$ & $124.3(6)$ \\
\hline$C(19)-C(20)-C(21)$ & $123.4(6)$ \\
\hline $\mathrm{C}(19)-\mathrm{C}(20)-\mathrm{H}(20)$ & 118.3 \\
\hline $\mathrm{C}(21)-\mathrm{C}(20)-\mathrm{H}(20)$ & 118.3 \\
\hline $\mathrm{C}(20)-\mathrm{C}(21)-\mathrm{C}(12)$ & $117.0(6)$ \\
\hline $\mathrm{C}(20)-\mathrm{C}(21)-\mathrm{C}(1)$ & $134.3(6)$ \\
\hline$C(12)-C(21)-C(1)$ & $108.8(6)$ \\
\hline $\mathrm{C}(5)-\mathrm{C}(22)-\mathrm{H}(22 \mathrm{~A})$ & 109.5 \\
\hline $\mathrm{C}(5)-\mathrm{C}(22)-\mathrm{H}(22 \mathrm{~B})$ & 109.5 \\
\hline $\mathrm{H}(22 \mathrm{~A})-\mathrm{C}(22)-\mathrm{H}(22 \mathrm{~B})$ & 109.5 \\
\hline $\mathrm{C}(5)-\mathrm{C}(22)-\mathrm{H}(22 \mathrm{C})$ & 109.5 \\
\hline $\mathrm{H}(22 \mathrm{~A})-\mathrm{C}(22)-\mathrm{H}(22 \mathrm{C})$ & 109.5 \\
\hline $\mathrm{H}(22 \mathrm{~B})-\mathrm{C}(22)-\mathrm{H}(22 \mathrm{C})$ & 109.5 \\
\hline $\mathrm{C}(5)-\mathrm{C}(23)-\mathrm{H}(23 \mathrm{~A})$ & 109.5 \\
\hline $\mathrm{C}(5)-\mathrm{C}(23)-\mathrm{H}(23 \mathrm{~B})$ & 109.5 \\
\hline $\mathrm{H}(23 \mathrm{~A})-\mathrm{C}(23)-\mathrm{H}(23 \mathrm{~B})$ & 109.5 \\
\hline $\mathrm{C}(5)-\mathrm{C}(23)-\mathrm{H}(23 \mathrm{C})$ & 109.5 \\
\hline $\mathrm{H}(23 \mathrm{~A})-\mathrm{C}(23)-\mathrm{H}(23 \mathrm{C})$ & 109.5 \\
\hline $\mathrm{H}(23 \mathrm{~B})-\mathrm{C}(23)-\mathrm{H}(23 \mathrm{C})$ & 109.5 \\
\hline $\mathrm{C}(8)-\mathrm{C}(24)-\mathrm{H}(24 \mathrm{~A})$ & 109.5 \\
\hline $\mathrm{C}(8)-\mathrm{C}(24)-\mathrm{H}(24 \mathrm{~B})$ & 109.5 \\
\hline $\mathrm{H}(24 \mathrm{~A})-\mathrm{C}(24)-\mathrm{H}(24 \mathrm{~B})$ & 109.5 \\
\hline $\mathrm{C}(8)-\mathrm{C}(24)-\mathrm{H}(24 \mathrm{C})$ & 109.5 \\
\hline $\mathrm{H}(24 \mathrm{~A})-\mathrm{C}(24)-\mathrm{H}(24 \mathrm{C})$ & 109.5 \\
\hline $\mathrm{H}(24 \mathrm{~B})-\mathrm{C}(24)-\mathrm{H}(24 \mathrm{C})$ & 109.5 \\
\hline $\mathrm{C}(8)-\mathrm{C}(25)-\mathrm{H}(25 \mathrm{~A})$ & 109.5 \\
\hline $\mathrm{C}(8)-\mathrm{C}(25)-\mathrm{H}(25 \mathrm{~B})$ & 109.5 \\
\hline $\mathrm{H}(25 \mathrm{~A})-\mathrm{C}(25)-\mathrm{H}(25 \mathrm{~B})$ & 109.5 \\
\hline $\mathrm{C}(8)-\mathrm{C}(25)-\mathrm{H}(25 \mathrm{C})$ & 109.5 \\
\hline $\mathrm{H}(25 \mathrm{~A})-\mathrm{C}(25)-\mathrm{H}(25 \mathrm{C})$ & 109.5 \\
\hline $\mathrm{H}(25 \mathrm{~B})-\mathrm{C}(25)-\mathrm{H}(25 \mathrm{C})$ & 109.5 \\
\hline $\mathrm{C}(15)-\mathrm{C}(26)-\mathrm{H}(26 \mathrm{~A})$ & 109.3 \\
\hline $\mathrm{C}(15)-\mathrm{C}(26)-\mathrm{H}(26 \mathrm{~B})$ & 109.6 \\
\hline $\mathrm{H}(26 \mathrm{~A})-\mathrm{C}(26)-\mathrm{H}(26 \mathrm{~B})$ & 109.5 \\
\hline $\mathrm{C}(15)-\mathrm{C}(26)-\mathrm{H}(26 \mathrm{C})$ & 109.5 \\
\hline $\mathrm{H}(26 \mathrm{~A})-\mathrm{C}(26)-\mathrm{H}(26 \mathrm{C})$ & 109.5 \\
\hline $\mathrm{H}(26 \mathrm{~B})-\mathrm{C}(26)-\mathrm{H}(26 \mathrm{C})$ & 109.5 \\
\hline $\mathrm{C}(15)-\mathrm{C}(27)-\mathrm{H}(27 \mathrm{~A})$ & 110.7 \\
\hline $\mathrm{C}(15)-\mathrm{C}(27)-\mathrm{H}(27 \mathrm{~B})$ & 108.7 \\
\hline $\mathrm{H}(27 \mathrm{~A})-\mathrm{C}(27)-\mathrm{H}(27 \mathrm{~B})$ & 109.5 \\
\hline $\mathrm{C}(15)-\mathrm{C}(27)-\mathrm{H}(27 \mathrm{C})$ & 109.0 \\
\hline $\mathrm{H}(27 \mathrm{~A})-\mathrm{C}(27)-\mathrm{H}(27 \mathrm{C})$ & 109.5 \\
\hline $\mathrm{H}(27 \mathrm{~B})-\mathrm{C}(27)-\mathrm{H}(27 \mathrm{C})$ & 109.5 \\
\hline $\mathrm{C}(18)-\mathrm{C}(28)-\mathrm{H}(28 \mathrm{~A})$ & 109.5 \\
\hline $\mathrm{C}(18)-\mathrm{C}(28)-\mathrm{H}(28 \mathrm{~B})$ & 109.5 \\
\hline $\mathrm{H}(28 \mathrm{~A})-\mathrm{C}(28)-\mathrm{H}(28 \mathrm{~B})$ & 109.5 \\
\hline $\mathrm{C}(18)-\mathrm{C}(28)-\mathrm{H}(28 \mathrm{C})$ & 109.5 \\
\hline $\mathrm{H}(28 \mathrm{~A})-\mathrm{C}(28)-\mathrm{H}(28 \mathrm{C})$ & 109.5 \\
\hline $\mathrm{H}(28 \mathrm{~B})-\mathrm{C}(28)-\mathrm{H}(28 \mathrm{C})$ & 109.5 \\
\hline $\mathrm{C}(18)-\mathrm{C}(29)-\mathrm{H}(29 \mathrm{~A})$ & 109.5 \\
\hline C(18)-C(29)-H(29B) & 109.5 \\
\hline $\mathrm{H}(29 \mathrm{~A})-\mathrm{C}(29)-\mathrm{H}(29 \mathrm{~B})$ & 109.5 \\
\hline $\mathrm{C}(18)-\mathrm{C}(29)-\mathrm{H}(29 \mathrm{C})$ & 109.5 \\
\hline $\mathrm{H}(29 \mathrm{~A})-\mathrm{C}(29)-\mathrm{H}(29 \mathrm{C})$ & 109.5 \\
\hline $\mathrm{H}(29 B)-C(29)-H(29 C)$ & 109.5 \\
\hline $\mathrm{Si}(1)-\mathrm{C}(30)-\mathrm{H}(30 \mathrm{~A})$ & 109.5 \\
\hline $\mathrm{Si}(1)-\mathrm{C}(30)-\mathrm{H}(30 \mathrm{~B})$ & 109.5 \\
\hline $\mathrm{H}(30 \mathrm{~A})-\mathrm{C}(30)-\mathrm{H}(30 \mathrm{~B})$ & 109.5 \\
\hline $\mathrm{Si}(1)-\mathrm{C}(30)-\mathrm{H}(30 \mathrm{C})$ & 109.5 \\
\hline $\mathrm{H}(30 \mathrm{~A})-\mathrm{C}(30)-\mathrm{H}(30 \mathrm{C})$ & 109.5 \\
\hline $\mathrm{H}(30 \mathrm{~B})-\mathrm{C}(30)-\mathrm{H}(30 \mathrm{C})$ & 109.5 \\
\hline $\mathrm{Si}(1)-\mathrm{C}(31)-\mathrm{H}(31 \mathrm{~A})$ & 109.5 \\
\hline $\mathrm{Si}(1)-\mathrm{C}(31)-\mathrm{H}(31 \mathrm{~B})$ & 109.5 \\
\hline
\end{tabular}




\begin{tabular}{|c|c|c|c|}
\hline $\mathrm{H}(31 \mathrm{~A})-\mathrm{C}(31)-\mathrm{H}(31 \mathrm{~B})$ & 109.5 & $\mathrm{H}(35 \mathrm{~A})-\mathrm{C}(35)-\mathrm{H}(35 \mathrm{C})$ & 109.5 \\
\hline $\mathrm{H}(31 \mathrm{~B})-\mathrm{C}(31)-\mathrm{H}(31 \mathrm{C})$ & 109.5 & $\mathrm{O}(1)-\mathrm{C}(36)-\mathrm{H}(36 \mathrm{~A})$ & 108.8 \\
\hline $\mathrm{N}(1)-\mathrm{C}(32)-\mathrm{C}(34)$ & $108.8(5)$ & $\mathrm{O}(1)-\mathrm{C}(36)-\mathrm{H}(36 \mathrm{~B})$ & 108.8 \\
\hline$C(33)-C(32)-C(34)$ & $108.3(5)$ & $\mathrm{C}(37)-\mathrm{C}(36)-\mathrm{H}(36 \mathrm{~B})$ & 108.8 \\
\hline $\mathrm{N}(1)-\mathrm{C}(32)-\mathrm{C}(35)$ & $108.2(5)$ & $\mathrm{H}(36 \mathrm{~A})-\mathrm{C}(36)-\mathrm{H}(36 \mathrm{~B})$ & 107.7 \\
\hline $\mathrm{C}(32)-\mathrm{C}(33)-\mathrm{H}(33 \mathrm{~A})$ & 109.5 & $\mathrm{H}(37 \mathrm{~A})-\mathrm{C}(37)-\mathrm{H}(37 \mathrm{~B})$ & 109.5 \\
\hline C(32)-C(33)-H(33B) & 109.5 & $\mathrm{C}(36)-\mathrm{C}(37)-\mathrm{H}(37 \mathrm{C})$ & 109.5 \\
\hline $\mathrm{H}(33 \mathrm{~A})-\mathrm{C}(33)-\mathrm{H}(33 \mathrm{~B})$ & 109.5 & $\mathrm{H}(37 \mathrm{~A})-\mathrm{C}(37)-\mathrm{H}(37 \mathrm{C})$ & 109.5 \\
\hline $\mathrm{C}(32)-\mathrm{C}(33)-\mathrm{H}(33 \mathrm{C})$ & 109.5 & $\mathrm{H}(37 \mathrm{~B})-\mathrm{C}(37)-\mathrm{H}(37 \mathrm{C})$ & 109.5 \\
\hline $\mathrm{H}(33 \mathrm{~A})-\mathrm{C}(33)-\mathrm{H}(33 \mathrm{C})$ & 109.5 & $\mathrm{C}(39)-\mathrm{C}(38)-\mathrm{O}(1)$ & $109.5(9)$ \\
\hline $\mathrm{C}(32)-\mathrm{C}(34)-\mathrm{H}(34 \mathrm{C})$ & 109.5 & $\mathrm{H}(38 \mathrm{~A})-\mathrm{C}(38)-\mathrm{H}(38 \mathrm{~B})$ & 108.2 \\
\hline $\mathrm{H}(34 \mathrm{~A})-\mathrm{C}(34)-\mathrm{H}(34 \mathrm{C})$ & 109.5 & $\mathrm{C}(38)-\mathrm{C}(39)-\mathrm{H}(39 \mathrm{~A})$ & 109.5 \\
\hline $\mathrm{H}(34 \mathrm{~B})-\mathrm{C}(34)-\mathrm{H}(34 \mathrm{C})$ & 109.5 & $\mathrm{C}(38)-\mathrm{C}(39)-\mathrm{H}(39 \mathrm{~B})$ & 109.5 \\
\hline $\mathrm{C}(32)-\mathrm{C}(35)-\mathrm{H}(35 \mathrm{~A})$ & 109.5 & $\mathrm{H}(39 \mathrm{~A})-\mathrm{C}(39)-\mathrm{H}(39 \mathrm{~B})$ & 109.5 \\
\hline $\mathrm{C}(32)-\mathrm{C}(35)-\mathrm{H}(35 \mathrm{~B})$ & 109.5 & $\mathrm{C}(38)-\mathrm{C}(39)-\mathrm{H}(39 \mathrm{C})$ & 109.5 \\
\hline $\mathrm{H}(35 \mathrm{~A})-\mathrm{C}(35)-\mathrm{H}(35 \mathrm{~B})$ & 109.5 & $\mathrm{H}(39 \mathrm{~A})-\mathrm{C}(39)-\mathrm{H}(39 \mathrm{C})$ & 109.5 \\
\hline $\mathrm{C}(32)-\mathrm{C}(35)-\mathrm{H}(35 \mathrm{C})$ & 109.5 & $\mathrm{H}(39 \mathrm{~B})-\mathrm{C}(39)-\mathrm{H}(39 \mathrm{C})$ & 109.5 \\
\hline
\end{tabular}

Table 13. Anisotropic displacement parameters $\left(\AA^{2} \times 10^{3}\right)$ for sm68. The anisotropic displacement factor exponent takes the form: $-2 \mathrm{p}^{2}\left[\mathrm{~h}^{2} \mathrm{a}^{* 2} \mathrm{U}^{11}+\ldots+2 \mathrm{hk} \mathrm{a}^{*} \mathrm{~b}^{*} \mathrm{U}^{12}\right]$

\begin{tabular}{|c|c|c|c|c|c|c|}
\hline & $\mathrm{U}^{11}$ & $\mathrm{U}^{22}$ & $\mathrm{U}^{33}$ & $\mathrm{U}^{23}$ & $\mathrm{U}^{13}$ & $\mathrm{U}^{12}$ \\
\hline $\operatorname{Zr}(1)$ & $17(1)$ & $16(1)$ & $21(1)$ & $0(1)$ & $6(1)$ & $1(1)$ \\
\hline $\operatorname{Si}(1)$ & $25(1)$ & $16(1)$ & $26(1)$ & $3(1)$ & $12(1)$ & $-2(1)$ \\
\hline $\mathrm{O}(1)$ & $26(3)$ & $30(3)$ & $24(3)$ & $7(2)$ & $-10(2)$ & $-7(3)$ \\
\hline $\mathrm{N}(1)$ & $22(3)$ & $17(3)$ & $18(3)$ & $0(2)$ & $6(2)$ & $1(3)$ \\
\hline $\mathrm{Cl}(1)$ & $32(1)$ & $37(1)$ & $30(1)$ & $1(1)$ & $2(1)$ & $-10(1)$ \\
\hline $\mathrm{C}(2)$ & $21(4)$ & $20(4)$ & $10(3)$ & $-1(3)$ & $6(3)$ & $5(3)$ \\
\hline $\mathrm{C}(3)$ & $24(4)$ & $15(4)$ & $16(3)$ & $-1(3)$ & $10(3)$ & 1(3) \\
\hline C(4) & 11(4) & $20(4)$ & $15(3)$ & $-3(3)$ & $7(3)$ & $4(3)$ \\
\hline$C(5)$ & $19(4)$ & $21(4)$ & $25(4)$ & $0(3)$ & $0(3)$ & $0(3)$ \\
\hline $\mathrm{C}(6)$ & $16(5)$ & $43(5)$ & $43(5)$ & $13(4)$ & $4(4)$ & $9(4)$ \\
\hline$C(7)$ & $18(4)$ & $40(5)$ & $42(5)$ & $9(4)$ & $12(4)$ & $-1(4)$ \\
\hline $\mathrm{C}(11)$ & $14(4)$ & $13(4)$ & $17(3)$ & $0(3)$ & $9(3)$ & $2(3)$ \\
\hline$C(12)$ & $22(4)$ & $20(4)$ & $16(3)$ & $-4(3)$ & $5(3)$ & $-9(3)$ \\
\hline$C(13)$ & $18(4)$ & $13(4)$ & $22(4)$ & $-2(3)$ & $6(3)$ & $-5(3)$ \\
\hline$C(14)$ & $13(4)$ & $22(4)$ & $24(4)$ & $-4(3)$ & $6(3)$ & $-1(3)$ \\
\hline$C(15)$ & $11(4)$ & $19(4)$ & $46(4)$ & $6(3)$ & $7(3)$ & $6(3)$ \\
\hline$C(16)$ & $17(3)$ & $30(3)$ & $35(4)$ & $2(3)$ & $11(3)$ & $5(3)$ \\
\hline$C(17)$ & $17(3)$ & $30(3)$ & $35(4)$ & $2(3)$ & $11(3)$ & $5(3)$ \\
\hline$C\left(16^{\prime}\right)$ & $17(3)$ & $30(3)$ & $35(4)$ & $2(3)$ & $11(3)$ & $5(3)$ \\
\hline$C\left(17^{\prime}\right)$ & $17(3)$ & $30(3)$ & $35(4)$ & $2(3)$ & $11(3)$ & $5(3)$ \\
\hline $\mathrm{C}(18)$ & $15(4)$ & $23(4)$ & $43(4)$ & $-6(4)$ & $4(3)$ & $2(3)$ \\
\hline C(19) & $13(4)$ & $23(4)$ & $23(4)$ & $-4(3)$ & $4(3)$ & $1(3)$ \\
\hline $\mathrm{C}(20)$ & $25(5)$ & $14(4)$ & $28(4)$ & $-5(3)$ & $13(3)$ & $-7(3)$ \\
\hline
\end{tabular}




\begin{tabular}{llccccc}
$\mathrm{C}(23)$ & $32(5)$ & $46(5)$ & $23(4)$ & $3(4)$ & $1(4)$ & $6(4)$ \\
$\mathrm{C}(24)$ & $23(5)$ & $31(5)$ & $55(5)$ & $-11(4)$ & $20(4)$ & $-9(4)$ \\
$\mathrm{C}(25)$ & $30(5)$ & $54(6)$ & $32(4)$ & $7(4)$ & $8(4)$ & $-9(4)$ \\
$\mathrm{C}(26)$ & $57(6)$ & $28(5)$ & $63(5)$ & $2(4)$ & $23(5)$ & $18(4)$ \\
$\mathrm{C}(27)$ & $56(6)$ & $57(7)$ & $46(5)$ & $34(5)$ & $15(5)$ & $28(5)$ \\
$\mathrm{C}(28)$ & $19(6)$ & $126(10)$ & $89(7)$ & $25(7)$ & $24(5)$ & $-16(6)$ \\
$\mathrm{C}(29)$ & $16(6)$ & $110(10)$ & $138(10)$ & $-95(9)$ & $-6(6)$ & $2(6)$ \\
$\mathrm{C}(30)$ & $50(5)$ & $25(4)$ & $26(4)$ & $-6(3)$ & $15(4)$ & $-4(4)$ \\
$\mathrm{C}(31)$ & $31(5)$ & $33(5)$ & $34(4)$ & $0(4)$ & $16(4)$ & $-2(4)$ \\
$\mathrm{C}(32)$ & $25(4)$ & $7(4)$ & $29(4)$ & $5(3)$ & $1(3)$ & $2(3)$ \\
$\mathrm{C}(33)$ & $35(5)$ & $26(4)$ & $31(4)$ & $6(3)$ & $10(4)$ & $3(4)$ \\
$\mathrm{C}(34)$ & $44(5)$ & $9(4)$ & $37(4)$ & $-8(3)$ & $10(4)$ & $10(3)$ \\
$\mathrm{C}(35)$ & $32(5)$ & $14(4)$ & $42(4)$ & $5(3)$ & $2(4)$ & $2(3)$ \\
$\mathrm{C}(36)$ & $34(5)$ & $23(4)$ & $29(4)$ & $3(4)$ & $8(4)$ & $-13(4)$ \\
$\mathrm{C}(37)$ & $120(9)$ & $49(6)$ & $42(5)$ & $-20(4)$ & $53(6)$ & $-35(6)$ \\
$\mathrm{C}(38)$ & $92(9)$ & $34(6)$ & $82(7)$ & $18(5)$ & $22(7)$ & $4(6)$ \\
$\mathrm{C}(39)$ & $84(9)$ & $122(11)$ & $121(10)$ & $-62(9)$ & $26(8)$ & $17(8)$ \\
\hline
\end{tabular}

Table 14. Hydrogen coordinates ( $\left.x 10^{4}\right)$ and isotropic displacement parameters $\left(\AA^{2} \times 10^{3}\right.$ ) for sm68.

\begin{tabular}{|c|c|c|c|c|}
\hline & $\mathrm{x}$ & $\mathrm{y}$ & $\mathrm{z}$ & $\mathrm{U}(\mathrm{eq})$ \\
\hline $\mathrm{H}(3)$ & 1153 & -2208 & 3464 & 21 \\
\hline $\mathrm{H}(6 \mathrm{~A})$ & 3004 & -649 & 3796 & 42 \\
\hline $\mathrm{H}(6 \mathrm{~B})$ & 2553 & -651 & 2735 & 42 \\
\hline $\mathrm{H}(7 \mathrm{~A})$ & 2813 & 1198 & 3272 & 39 \\
\hline $\mathrm{H}(7 \mathrm{~B})$ & 2568 & 991 & 4133 & 39 \\
\hline $\mathrm{H}(10)$ & 761 & 1498 & 2297 & 18 \\
\hline $\mathrm{H}(13)$ & -396 & 1893 & 1827 & 21 \\
\hline $\mathrm{H}(16 \mathrm{~A})$ & -2410 & 2744 & 592 & 32 \\
\hline $\mathrm{H}(16 \mathrm{~B})$ & -2211 & 1673 & 101 & 32 \\
\hline $\mathrm{H}(17 \mathrm{~A})$ & -2859 & 1144 & 952 & 32 \\
\hline $\mathrm{H}(17 \mathrm{~B})$ & -2376 & 1566 & 1912 & 32 \\
\hline $\mathrm{H}(16 \mathrm{C})$ & -2197 & 2197 & 1913 & 32 \\
\hline $\mathrm{H}(16 \mathrm{D})$ & -2422 & 2784 & 885 & 32 \\
\hline $\mathrm{H}(17 \mathrm{C})$ & -2850 & 1012 & 693 & 32 \\
\hline H(17D) & -2352 & 954 & 202 & 32 \\
\hline $\mathrm{H}(20)$ & -1327 & -1505 & 2126 & 25 \\
\hline $\mathrm{H}(22 \mathrm{~A})$ & 2078 & -2510 & 2761 & 57 \\
\hline $\mathrm{H}(22 \mathrm{~B})$ & 2050 & -2922 & 3768 & 57 \\
\hline $\mathrm{H}(22 \mathrm{C})$ & 2669 & -2605 & 3652 & 57 \\
\hline $\mathrm{H}(23 \mathrm{~A})$ & 2728 & -1389 & 5100 & 53 \\
\hline $\mathrm{H}(23 \mathrm{~B})$ & 2052 & -1613 & 4982 & 53 \\
\hline $\mathrm{H}(23 \mathrm{C})$ & 2288 & -336 & 4990 & 53 \\
\hline $\mathrm{H}(24 \mathrm{~A})$ & 1464 & 2734 & 2738 & 52 \\
\hline $\mathrm{H}(24 \mathrm{~B})$ & 2161 & 2868 & 3208 & 52 \\
\hline $\mathrm{H}(24 \mathrm{C})$ & 1782 & 2352 & 3816 & 52 \\
\hline $\mathrm{H}(25 \mathrm{~A})$ & 1949 & 519 & 1587 & 59 \\
\hline $\mathrm{H}(25 \mathrm{~B})$ & 2188 & 1802 & 1762 & 59 \\
\hline $\mathrm{H}(25 \mathrm{C})$ & 1496 & 1559 & 1418 & 59 \\
\hline $\mathrm{H}(26 \mathrm{~A})$ & -1630 & 3817 & 1859 & 73 \\
\hline $\mathrm{H}(26 \mathrm{~B})$ & -1001 & 3298 & 2336 & 73 \\
\hline $\mathrm{H}(26 \mathrm{C})$ & -1539 & 2807 & 2582 & 73 \\
\hline $\mathrm{H}(27 \mathrm{~A})$ & -1367 & 2298 & -13 & 80 \\
\hline $\mathrm{H}(27 \mathrm{~B})$ & -912 & 3029 & 770 & 80 \\
\hline $\mathrm{H}(27 \mathrm{C})$ & -1548 & 3498 & 261 & 80 \\
\hline $\mathrm{H}(28 \mathrm{~A})$ & -2744 & -389 & 2089 & 115 \\
\hline $\mathrm{H}(28 \mathrm{~B})$ & -2142 & 115 & 2800 & 115 \\
\hline $\mathrm{H}(28 \mathrm{C})$ & -2167 & -1169 & 2424 & 115 \\
\hline $\mathrm{H}(29 \mathrm{~A})$ & -2169 & -1520 & 787 & 143 \\
\hline $\mathrm{H}(29 \mathrm{~B})$ & -2267 & -467 & 69 & 143 \\
\hline
\end{tabular}




\begin{tabular}{lrrrr}
$\mathrm{H}(29 \mathrm{C})$ & -2788 & -870 & 441 & 143 \\
$\mathrm{H}(30 \mathrm{~A})$ & 375 & -3248 & 5201 & 50 \\
$\mathrm{H}(30 \mathrm{~B})$ & 133 & -1965 & 5059 & 50 \\
$\mathrm{H}(30 \mathrm{C})$ & 726 & -2281 & 4852 & 50 \\
$\mathrm{H}(31 \mathrm{~A})$ & -1099 & -3481 & 2823 & 47 \\
$\mathrm{H}(31 \mathrm{~B})$ & -1066 & -2562 & 3633 & 47 \\
$\mathrm{H}(31 \mathrm{C})$ & -875 & -3856 & 3915 & 47 \\
$\mathrm{H}(33 \mathrm{~A})$ & 107 & -4988 & 4496 & 47 \\
$\mathrm{H}(33 \mathrm{~B})$ & 197 & -6263 & 4185 & 47 \\
$\mathrm{H}(33 \mathrm{C})$ & -357 & -5530 & 3564 & 47 \\
$\mathrm{H}(34 \mathrm{~A})$ & -57 & -5748 & 2071 & 46 \\
$\mathrm{H}(34 \mathrm{~B})$ & 379 & -6641 & 2766 & 46 \\
$\mathrm{H}(34 \mathrm{C})$ & 633 & -5688 & 2239 & 46 \\
$\mathrm{H}(35 \mathrm{~A})$ & 1281 & -4621 & 3597 & 47 \\
$\mathrm{H}(35 \mathrm{~B})$ & 1210 & -5743 & 4160 & 47 \\
$\mathrm{H}(35 \mathrm{C})$ & 1097 & -4512 & 4531 & 47 \\
$\mathrm{H}(36 \mathrm{~A})$ & 886 & -1055 & 1277 & 35 \\
$\mathrm{H}(36 \mathrm{~B})$ & 474 & -198 & 511 & 35 \\
$\mathrm{H}(37 \mathrm{~A})$ & 999 & -2183 & 7 & 96 \\
$\mathrm{H}(37 \mathrm{~B})$ & 1248 & -918 & 7 & 96 \\
$\mathrm{H}(37 \mathrm{C})$ & 621 & -1258 & -732 & 96 \\
$\mathrm{H}(38 \mathrm{~A})$ & -248 & -1419 & -846 & 85 \\
$\mathrm{H}(38 \mathrm{~B})$ & -670 & -2188 & -454 & 85 \\
$\mathrm{H}(39 \mathrm{~A})$ & -884 & -648 & 272 & 166 \\
$\mathrm{H}(39 \mathrm{~B})$ & -1050 & -443 & -846 & 166 \\
$\mathrm{H}(39 \mathrm{C})$ & -467 & 117 & -127 & 166 \\
\hline
\end{tabular}

